\title{
Magnetic tools for lab-on-a-chip technologies
}

\author{
by
}

Nikola Slobodan Pekas

\begin{abstract}
A dissertation submitted to the graduate faculty in partial fulfillment of the requirements for the degree of DOCTOR OF PHULOSOPHY
\end{abstract}

\author{
Major: Analytical Chemistry \\ Program of Study Committee: \\ Marc D. Porter, Major Professor \\ Edward S. Yeung \\ Surya K. Mallapragada \\ Joseph W. Burnett \\ Balaji Narasimhan
}

Iowa State University

Ames, Iowa 


\section{Graduate College \\ Iowa State University}

This is to certify that the doctoral dissertation of

Nikola Slobodan Pekas

has met the dissertation requirements of Iowa State University

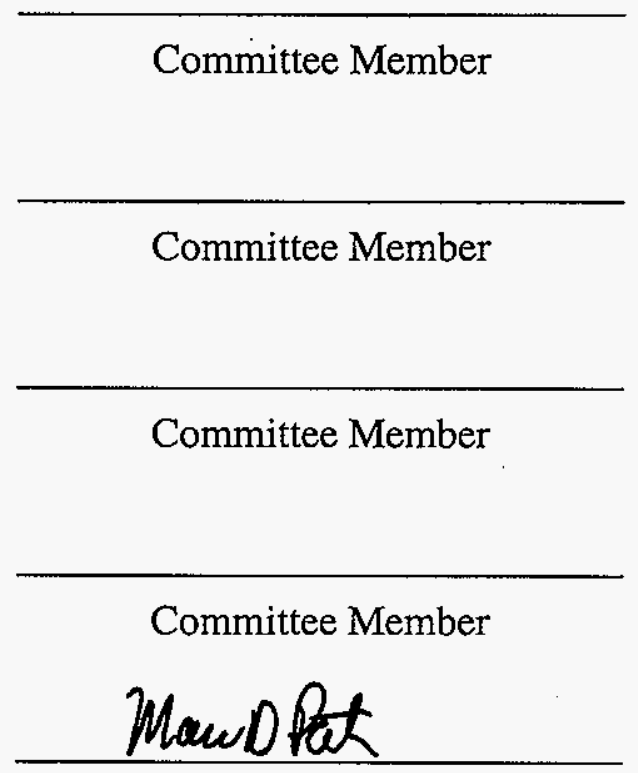

Major Professor

For the Major Program 


\section{TABLE OF CONTENTS}

ABSTRACT

ACKNOWLEDGEMENTS $\quad$ vi

CHAPTER 1. GENERAL INTRODUCTION

Dissertation organization and contribution credits 1

Laboratory on a chip $\quad 2$

Miniaturized Flow cytometry 6

Flow cytometry overview 6

Miniaturizing flow cytometry . 7

Overview of magnetism and magnetic particles 9

$\begin{array}{lr}\text { Magnetism } & 9\end{array}$

Structure and applications of magnetic particles $\quad 14$

Overview of giant magnetoresistance technologies 17

$\begin{array}{ll}\text { References cited } & 20\end{array}$

CHAPTER 2. MAGNETIC PARTICLE DIVERTER IN AN INTEGRATED MICROFLUIDIC FORMAT

Abstract

Introduction

Fabrication

29

Results and Discussion $\quad 31$

Conclusions $\quad 35$

$\begin{array}{ll}\text { References cited } & 35\end{array}$

CHAPTER 3. GIANT MAGNETORESISTANCE MONITORING OF MAGNETIC PICODROPLETS IN AN INTEGRATED MICROFLUIDIC SYSTEM

Abstract $\quad 37$

$\begin{array}{ll}\text { Introduction } & 37\end{array}$

Fabrication and experimental setup - 38

Results and discussion $\quad 40$

$\begin{array}{ll}\text { References cited } & 43\end{array}$

CHAPTER 4. QUANTITATIVE MAGNETIC SENSING IN MICROCHANNELS:

FIGURES OF MERIT AND IMPLICATIONS FOR MAGNETIC FLOW

CYTOMETRY

Abstract

44

Introduction

44

Experimental setup and numerical models

Results and discussion $\quad 48$

Quantitative measurements 48

Finite-element modeling of magnetic targets of interest

Detection of ferromagnetic beads in flow 55

Conclusions $\quad 58$

Acknowledgements $\quad 59$

References cited $\quad 59$ 
CHAPTER 5. TOWARD MAGNETIC FLOW CYTOMETRY ON A CHIP: DETECTION OF MAGNETOTACTIC BACTERIA IN A MICROFLUIDIC SYSTEM WITH EMBEDDED MAGNETORESISTIVE SENSORS

Abstract

Introduction

Design considerations

Fabrication

Device evaluation

Experiments

Determination of optimal volumetric flow rates

Detection of magnetotactic bacteria

Conclusions

Acknowledgements

References cited

CHAPTER 6. GENERAL CONCLUSIONS AND SUGGESTIONS FOR FURTHER RESEARCH

Device fabrication

The role of intrinsic fields

Hydrodynamic lift forces

Magnetic properties of labels, and magnetic and viscous torque 


\begin{abstract}
This study establishes a set of magnetics-based tools that have been integrated with microfluidic systems. The overall impact of the work begins to enable the rapid and efficient manipulation and detection of magnetic entities such as particles, picoliter-sized droplets, or bacterial cells. Details of design, fabrication, and theoretical and experimental assessments are presented. The manipulation strategy has been demonstrated in the format of a particle diverter, whereby micron-sized particles are actively directed into desired flow channels at a split-flow junction by means of integrated microelectromagnets. Magnetic detection has been realized by deploying Giant Magnetoresistance (GMR) sensors - microfabricated structures originally developed for use as readout elements in computer hard-drives. We successfully transferred the GMR technology to the lab-on-a-chip arena, and demonstrated the versatility of the concept in several important areas: real-time, integrated monitoring of the properties of multiphase droplet flows; rapid quantitative determination of the concentration of magnetic nanoparticles in droplets of ferrofluids; and high-speed detection of individual magnetic microparticles and magnetotactic bacteria. The study also includes novel schemes for. hydrodynamic flow focusing that work in conjunction with GMR-based detection to ensure precise navigation of the sample stream through the GMR detection volume, therefore effectively establishing a novel concept of a microfabricated magnetic flow cytometer.
\end{abstract}




\section{ACKNOWLEDGEMENTS}

Firstly, I wish to express my gratitude to Marc D. Porter for his patient guidance, contagious enthusiasm, and, above everything, for constantly confirming that it is possible to be serious about things, and yet be able to laugh at them. Next, I thank all the members of Porter group who accompanied me during my graduate studies. Among them, I am particularly thankful to Hajime Takano for his inspiring presence and friendship throughout the first two years of my studies. Robert T. Doyle provided a wonderful environment to work in, and answered many of my questions. My very special thankfulness goes to Rebecca L. Staedtler for all the logistic support and for her tolerance. It is impossible to list all the friends who, with their care and creativity, made the years of my journey towards doctorate such a pleasant and enriching experience. I am forever grateful to them.

This work was performed at Ames Laboratory under Contract No. W-7405-Eng-82 with the U.S. Department of Energy. The United States government has assigned the DOE Report number IS-T 1942 to this thesis. I also gratefully acknowledge the graduate fellowship support from ConocoPhillips.

Most importantly, I dedicate this degree to my parents, Ružica Bocić-Pekas and Slobodan Pekas. Their unconditional love has always been present to remind me what the important things in life are. 


\section{CHAPTER 1. GENERAL INTRODUCTION}

\section{Dissertation organization and contribution credits}

The dissertation is organized in six chapters. Chapter 1 includes a general introduction to the area of microminiaturized chemical and bioanalytical instrumentation, with an emphasis on aspects and technologies relevant to the experimental and theoretical work described in the following chapters: flow cytometry, magnetic micro- and nanoparticles, and microfabricated magnetic sensors.

Chapter 2 has been published in Journal of Magnetism and Magnetic Materials, and portrays a proof-of-concept demonstration of manipulation of magnetic microparticles in flow. The chips used were conceived and designed by Michael Granger of ISU and Mark Tondra of NVE Corporation, prior to my arrival to ISU. Micropatterning of aluminum current lines and fluidic channels has been performed at NVE by Anthony Popple and Mark Tondra. My work included the design and fabrication of elastomeric lids, optimization of the procedure for bonding the lids to the chips, building of the current-control module and a pumping system, modeling and fabrication of the external magnetic excitation structure, and finally designing and carrying out the experiments, data processing, and development of numerical models in MATLAB programming language.

Chapter 3 has been published in Applied Physics Letters; it describes a microfluidic system with embedded magnetic sensors. The system is used to generate in a controlled fashion magnetic droplets of the micrometer size, and to monitor the sensor response to the ensuing magnetically discrete flow pattern. The magnetic sensors were designed by Mark Tondra and fabricated under his supervision at NVE by Anthony Popple. A handy Magnetic Excitation and Data Acquisition (MEDA) module used to collect data has been developed by Albrecht Jander and Mark Tondra, along with the accompanying software. I have designed and fabricated the overlaying microfluidic structure in elastomeric lids, assembled devices, designed and carried out the described measurements, and processed data. Numerical models have been developed jointly by Mark Tondra, who built the starting AMPERES model, and myself, who wrote the MATLAB code to process and visualize the raw AMPERES data, and also to execute parametric simulations of the sensor response.

Chapter 4 presents quantitative measurements performed using the same platform as described in Chapter 3, finite-element modeling, and detailed discussion of the results and implications for the magnetic flow cytometry concept. Detection of individual magnetic particles in flow is also described. Experiments are based on contributions from the same people as in Chapter 3. I have developed the FEMLAB models, and processed the data. 
Chapter 5 discusses design, fabrication and assessment of a chip-scale magnetic flow cytometer. Magnetic sensors have been conceived and fabricated by Mark Tondra and Anthony Popple. I have designed the overlaid two-layer microfluidic architecture. The first fluidic layer was fabricated at NVE Corporation by Anthony Popple. I have fabricated the upper fluidic layer with the fluidic ports, and assembled the devices. Magnetotactic bacteria were cultured and fixed by Dennis Bazylinski of Iowa State University. I have designed and carried out the experiments, and processed the collected data, and constructed numerical models.

Chapter 6 gives general conclusions stemming from the entirety of the work presented in this dissertation, along with several recommendations for further work.

\section{Laboratory on a chip}

Miniaturization trends in the 20th century have been most obvious in the realm of electronic components and electronic integrated systems. ${ }^{1,2}$ Ensuing advances in microfabrication technologies had soon started being applied to other fields, such as pressure sensors and accelerometers, enabling development of complex MicroElectroMechanical Systems (MEMS), and, at the turn of the century, microfabricated paraphernalia for chemical or biological analysis and synthesis, so-called labs-on-chips (LOCs). Figures 1 and 2 show examples of microelectronic and microfluidic integrated systems, respectively.

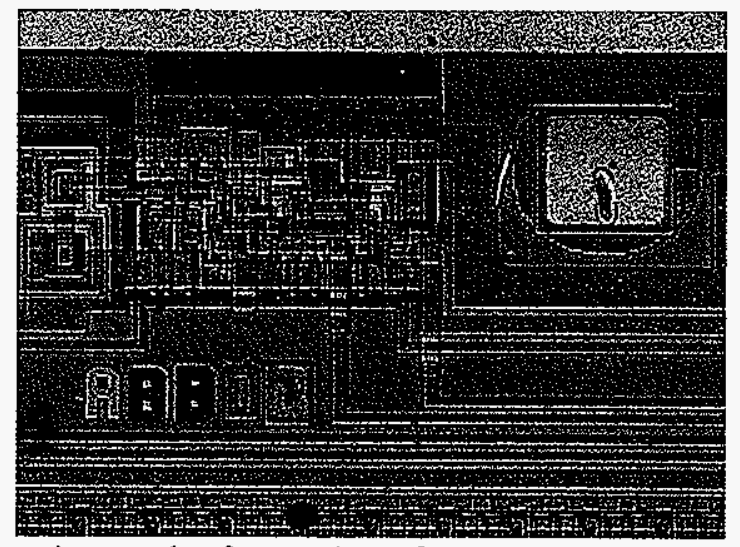

Figurel. A scanning electron micrograph of a portion of a semi-conductor device showing several printed circuits. Each color represents a different material. Adapted from Wikipedia. The image is in the public domain. 


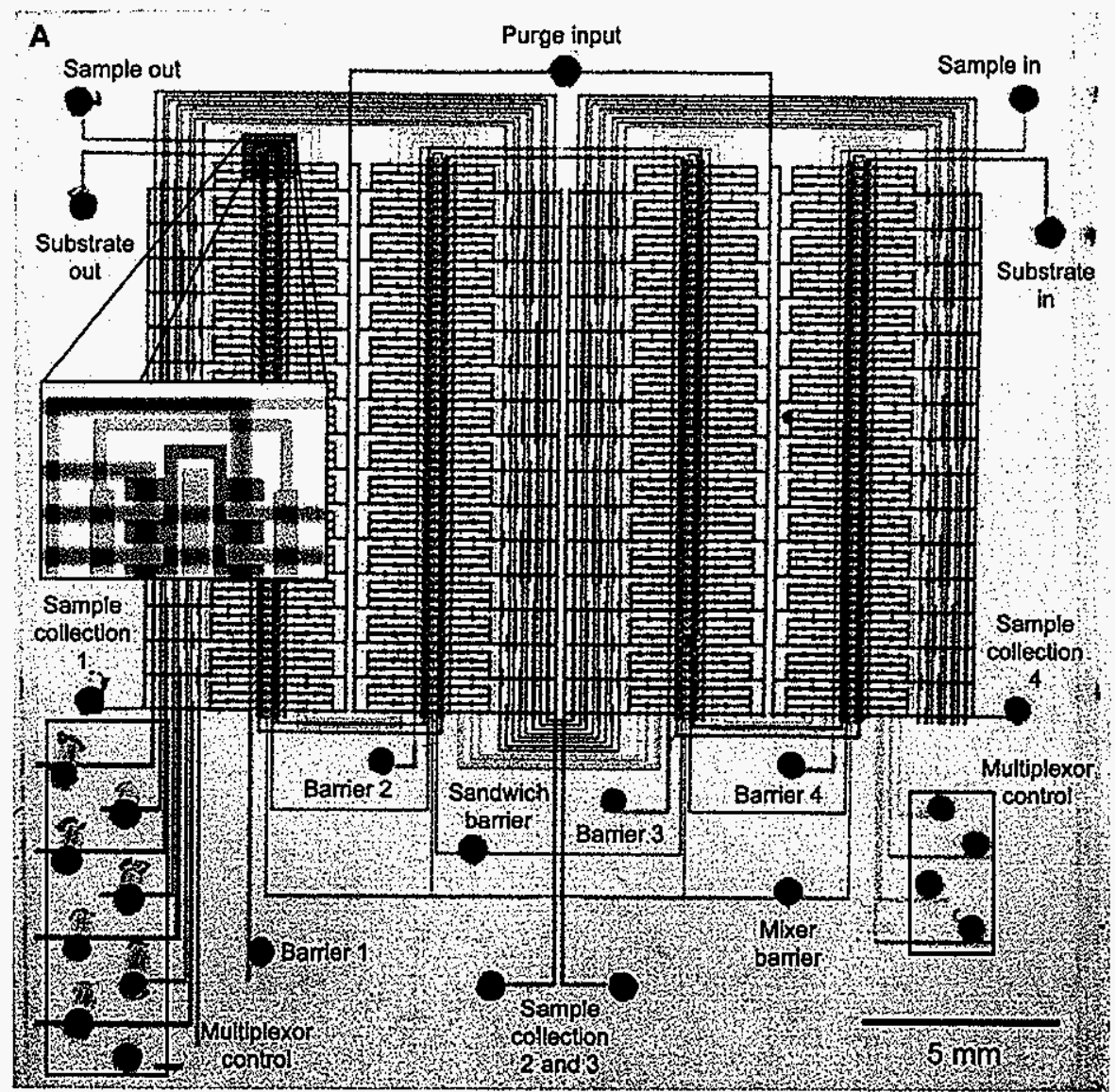

Figure 2. Optical micrograph of the microfluidic comparator chip. The various inputs have been loaded with food dyes to visualize the channels and subelements of the fluidic logic. ${ }^{3}$

The driving force behind the miniaturization - whether for information or material/energy processing - is the aspiration to increase the processing power, while reducing the economic cost (and, only recently, environmental impact). Two important consequences arise from this markedly exponential development. First, the increase in processing power brings about occasional paradigm leaps, enabling undertakings not possible before: numerical modeling of complex systems, minimally invasive surgeries, high throughput biochemical screening - to name a few current examples. Second, the decrease in production cost generally makes these capabilities more accessible across the socio-economic domain, as in personal computers, glucose monitors, or pregnancy tests.

Labs-on-chips generally speed up the reaction times, allow for massively parallel design, and are field deployable. Moreover, reagent and energy consumption are dramatically reduced, and so is the amount of waste produced. It should be noted, however, that the massive fabrication of inexpensive and disposable LOCs may pose an environmental problem simply due to the sheer volume of production - a problem already faced with many 
other miniaturized technologies: nickel-cadmium batteries, lead in electronic circuit boards, and mobile phones.

The first LOC analytical platform was a gas chromatograph, described by S.C. Terry in $1975 .{ }^{4,5}$ Since then, LOCs have been developed that handle liquids, ${ }^{6}$ gases, ${ }^{7}$ plasma, ${ }^{8-10}$ dry powders, ${ }^{11}$ and multi-phase liquid-liquid, ${ }^{12-15}$ gas-liquid, ${ }^{16}$ and liquid-solid ${ }^{17-21}$ systems. The range of applications can suitably be divided into three major categories:

1. Micro total-analysis systems ( $\mu \mathrm{TAS}$ ), for analyte identification and/or quantification purposes - this concept was the main premise of microfluidic systems in the early 1990s, and has since emerged into a variety of diagnostic, environmental, and military applications.

2. Microreactors for chemical synthesis or energy production - this field started in the late 1990s, and embodies methods to synthesize unstable, precious, or dangerous materials on demand; tailored nanoparticles ${ }^{15}$ and nanopatterned surfaces; $;^{22}$ or to develop micropower sources. ${ }^{23,24}$

3. Microfluidic tools for purposes ranging from screening for protein crystallization conditions $^{13}$ to interacting with single cells ${ }^{19,25-27}$ - these strategies take the advantage of physical and chemical properties that are unique to microfluidic systems (e.g. laminar flow, high surface-to-volume ratio, or feature sizes comparable to the size of cells), thus enabling experiments that would be very hard or even impossible to realize in the conventional, bench top format.

Large-scale integration and batch fabrication have led to commercial release of several LOCs, used mostly in the pharmaceutical and biotechnology markets. Examples include companies such as Fluidigm, ${ }^{21,28}$ Gyros, $^{29}$ and Agilent. ${ }^{30}$

An extensive list of principal and optional modules of an analytical LOC, for instance, would include sample preparation and enrichment, pumping/manipulation, separation, detection, signal conditioning, temperature control, and power. Due to either lack of fully developed modules in the micro-format, or integration challenges, many (if not most) of the listed functionalities are at the present time outsourced to the macro-world, thereby often diminishing the reputed advantages of the LOC concept.

Detection is clearly one of the key features in analytical LOC platforms, but also plays a potentially important role as a part of process control in synthetic and enabling microdevices. To date, a variety of detection strategies have been deployed in the LOC arena, with different levels of integration and portability. Epifluorescence and confocal microscopes are generally available on research sites, and, as a result, habitually used in detection schemes. Conventional microscopy abolishes the portability and low-cost advantages of such LOCs, 
and a number of groups have been working on integrating optical excitation sources and detectors into LOCs. ${ }^{31-33}$ Electrochemical methods usually rely on microfabricated, integrated electrodes and therefore promise a higher level of portability. ${ }^{34,35}$

One emerging approach to detection is based on magnetic micro- or nanoparticles as reporters. Several technologies are available for readout in this case - SQUID microscopes ${ }^{36}$ and microcoil-NMR ${ }^{37,38}$ have been used to detect the presence of magnetic reporters on miniaturized platforms. The described approaches, however, are based on bulky, off-chip equipment. A hand-held device for quantification of magnetic particles bound in a lateral flow assay has been developed by Quantum Design ${ }^{39}$ - however, this inductive-coil based method only measures bulk magnetization of a relatively large test strip. Hall effect sensors have been used to detect droplets of dilute magnetic bead suspensions, ${ }^{40}$ although without integration in a microfluidic and/or bioassay environment. A promising alternative lies in the field of micron-sized giant magnetoresistive (GMR) sensors ${ }^{14,41-44}$ originally developed in the past decade for and by the computer hard-drive industry. GMRs are exceptionally attractive since they are inherently small, compatible with the present LOC microfabrication techniques, and directly produce electronic signals for further processing. As demonstrated by the hard-drive readout rates, they guarantee extremely fast detection. Furthermore, magnetic particles conjugated to biorecognition or inorganic agents are well established, generally commercially available, and their magnetic properties are usually stable over time and under the wide range of conditions. These latter properties bypass problems analogous to photobleaching of fluorescent labels. Most biological samples show essentially no magnetic background signal equivalent to autofluorescence or turbidity in optical methods. Finally, magnetic labeling opens up the additional option to manipulate analytes on chip by means of localized magnetic field gradients.

This dissertation describes contributions to the field of integration of micromagnetic manipulation and detection tools in a microfluidic environment. Our goal is to create field deployable, integrated systems capable of rapidly and accurately detecting and quantifying magnetically labeled bioanalytes or inherently magnetic targets, with GMR technology as a cornerstone. To date, this concept has been explored mostly in the form of surface-bound assay chips with DNA ${ }^{41,42}$ or antibody ${ }^{43}$ capture surfaces, and magnetic particles as reporters. The work presented herein develops flow-based microsystems for enumeration and manipulation of bioparticles in flow - magnetic flow cytometers. 


\section{Miniaturized Flow cytometry \\ Flow cytometry overview}

Flow cytometry is a powerful and well-established tool for high speed, multiparametric analysis of single cells. It is used in the fields of microbiology, biochemistry, and drug discovery, often coupled to a cell-sorting module, thus enabling not only enumeration and analysis, but also collection of desired subpopulations of cells. In a relatively recent innovative twist, flow cytometers have also been used as tools for high-throughput screening of liquid-suspended arrays of microspheres. ${ }^{45,46}$

In 1947, Gucker et al. described an apparatus for counting colloidal particles such as bacterial spores in aerosols, generally recognized as the first flow cytometer. ${ }^{47,48}$ In a typical modern flow cytometer, ${ }^{49,50}$ analyte particles (eukaryotic cells, bacteria, organelles, or beads) are suspended in a suitable aqueous buffer, and pumped through the flow chamber, where this stream is injected coaxially into a particle-free sheath stream. Thus, particles are individualized and hydrodynamically focused to pass precisely through the observation point in a filament flow.

The detection is usually realized by optical means, ${ }^{48-50}$ and is in some cases accompanied by a Coulter aperture for cell sizing. ${ }^{51-53}$ To guarantee reliable detection at the fast flow rates, the illumination source must provide sufficient power, which is in principle achieved by using either a high-pressure mercury lamp with appropriate filters, or lasers. The illumination and collecting optics include an array of mirrors, total reflection prisms, and multiple spherical, or cylindrical lenses. An example of an optical configuration is shown in Figure 3.49

When a particle passes through the detection volume, a short pulse of scattered light is produced. If particles are fluorescently labeled, a fluorescence pulse will accompany the scattered signal. Duration of pulses is typically in the range of 3-20 $\mu \mathrm{s}$, as dictated by the flow velocity, particle size, and the focus spot size. ${ }^{49}$ The signals are collected by several detection systems in parallel. Forward light scatter, collected in the range of 2-20 degrees off the illumination axis, is correlated roughly with the cell size. The side scatter signal, collected perpendicularly to the illumination axis, correlates with the internal structure or surface roughness. Fluorescence is also observed from the side, and bears highly specific information about the presence of target antigens or nucleotide sequences, as probed by the fluorescent biorecognition agents used. The orthogonal arrangement of the sample flow, illumination, and detection places a high level of demand on the alignment and calibration of the system. 

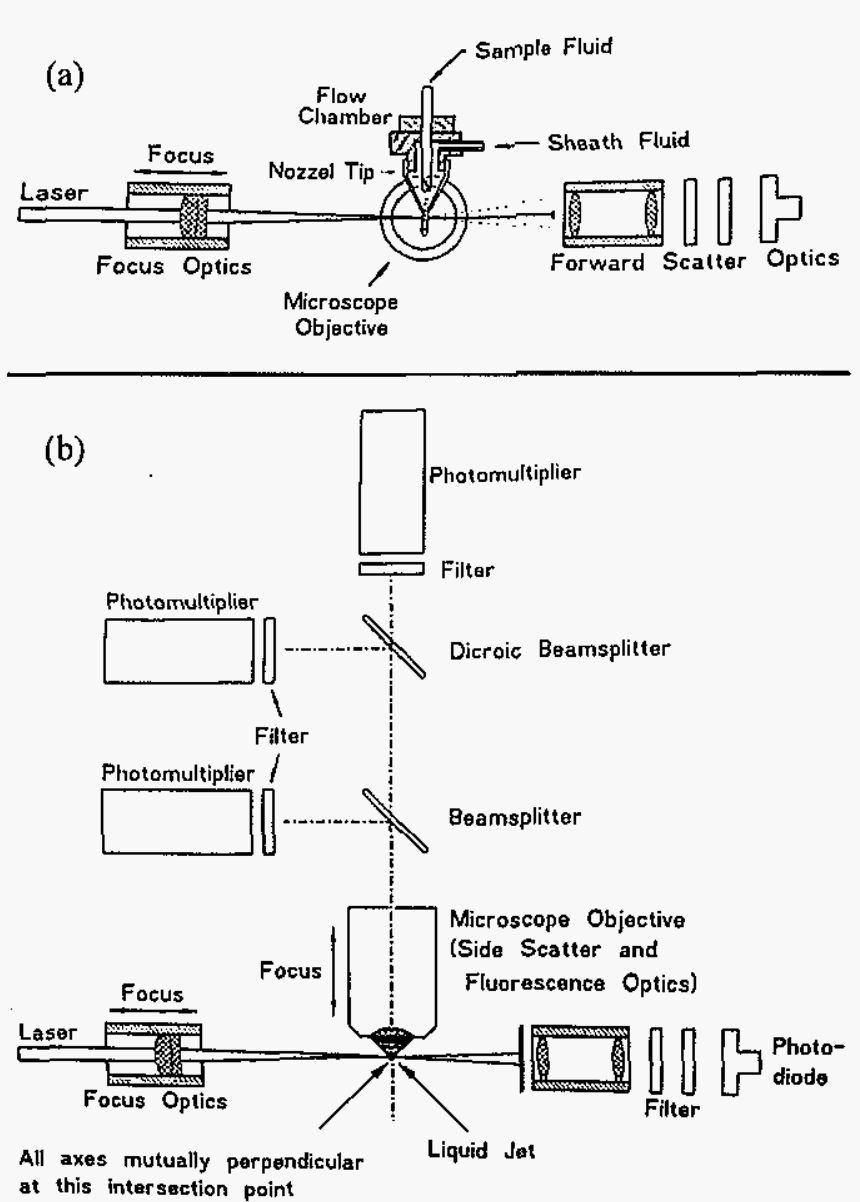

Figure 3. Side view (a) and top view (b) of a flow cytometry optical configuration. ${ }^{49}$

Forward light scatter signal is detected with a photodiode, whereas side-scatter and fluorescence signals require more sensitive photomultiplier tubes. Furthermore, if multiple fluorescence channels are desired, the fluorescence light is passed through a series of dichroic beamsplitters and filters, directing signal components to the proper photomultiplier tubes. Electrical signals from the detectors are passed through either linear or logarithmic amplifiers, and digitized for further processing and storage.

\section{Miniaturizing flow cytometry}

In the context of benefits of miniaturization discussed earlier, efforts have been present to devise microminiaturized flow cytometers and sorters ( $\mu$ FCM). Most of the work published to date involves microfabricated flow cells accompanied by off-chip, conventional detection and sorting modules - excitation lasers, photomultiplier tubes, and pumps. Despite nonportability, such systems still offer advantages over conventional flow cytometers: they reduce consumption of reagents, enable more efficient handling of cells available in limited 
supply, minimize sample carryover, and facilitate safe handling of biohazard samples. Wang et al. have described a fluorescence-activated cell sorter based on a glass microfluidic chip, an external 488-nm semiconductor laser for excitation, a photomultiplier tube for fluorescence detection, and a near-infrared laser for cell deflection. ${ }^{54} \mathrm{~A}$ microcytometry platform in a "lab-on-a-card" format is now available from Micronics, ${ }^{55}$ and is shown in Figure 4. The system utilizes laser-patterned thin plastic sheets stacked together to form a multilayered fluidic structure, roughly of the credit-card size. The card includes modules for in situ fluorescent labeling of cells by interfacial diffusion, and cell sorting. It is accompanied by a desktop pumping module, and fits onto a microscope stage for CCD-based detection and cell enumeration.

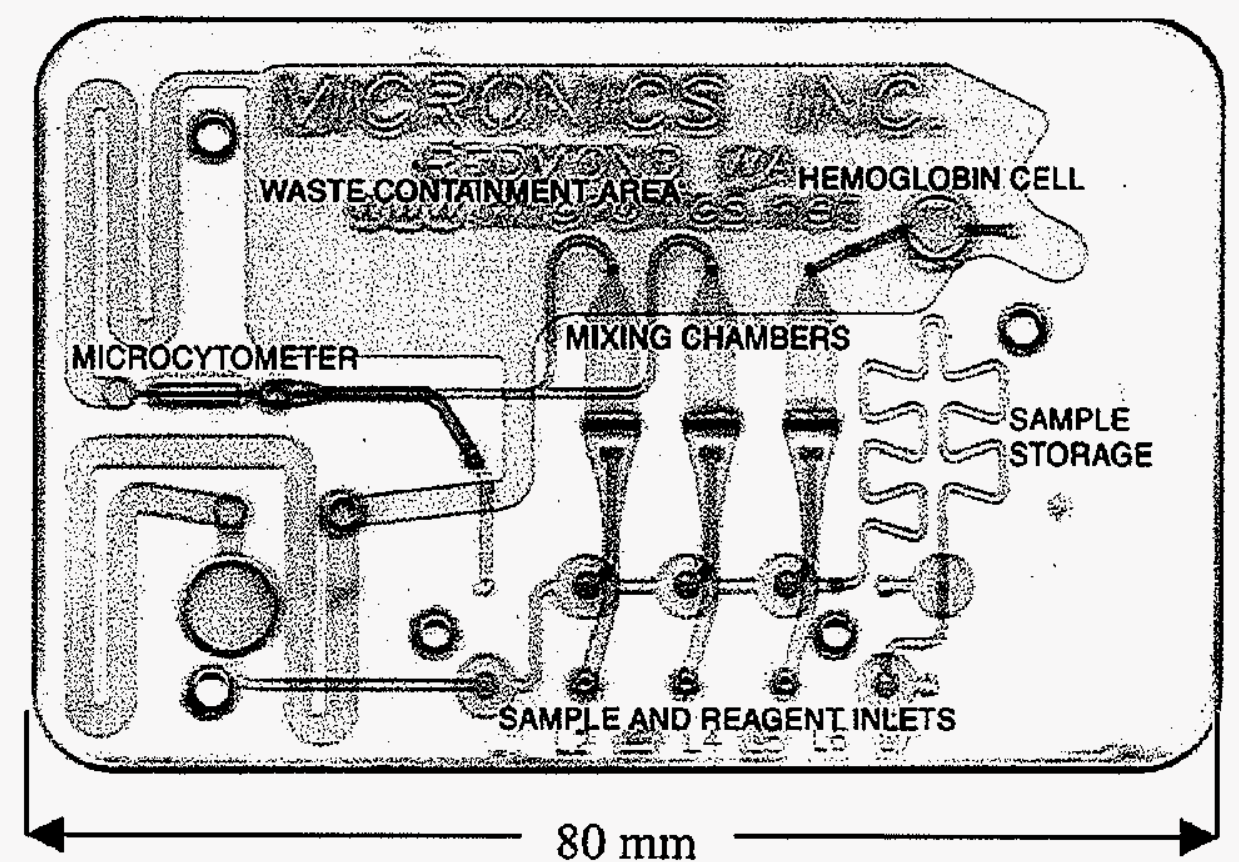

Figure 4. Micronics microcytometry system realized in a plastic laminate card format.

Agilent has introduced the lab-chip analyzer, ${ }^{56}$ currently capable of two-channel fluorescence automated analysis of up to six $10-\mu \mathrm{L}$ samples of prestained cells. This microfluidic chip, however, still requires a bench top support unit. Impedance spectroscopy has also been explored as a readout method, and offers potential for integration with microdevices. Platforms have been described based on arrays of microfabricated electrodes, ${ }^{57,58}$ capable of dielectric-properties discrimination based on the impedance measurements in the megahertz range. It is worth noting that cells are essentially nonconductive in the low-frequency range, and low-frequency or dc measurements therefore 
discriminate by cell size, simply due to the electrolyte volume displacement, as in Coulter counters. ${ }^{51,52}$ One way to perform on-chip dc measurements is to use polyelectrolyte salt bridges in place of metal electrodes. ${ }^{59}$

Both optical and electrical detection schemes have their limitations: the portrayed optical detection systems are based on external excitation sources and detectors, which not only sacrifices portability, but also imposes stringent requirements for optical alignment and vibration control. Impedance-based schemes, on the other hand, are relatively easy to implement on-chip, however, the lack of recognition-based labeling modalities results in little, if any, biolyte specificity.

\section{Prospects for magnetic $\mu$ FCM}

Integration of inherently small magnetic sensors promises a truly portable system for sensing of magnetically labeled analytes. Magnetic bioconjugate labels are widely available in sizes ranging from $50 \mathrm{~nm}$ to micrometers, owing mostly to the sample enrichment applications within the biomedical diagnostics market. Although the particle size lowers the rates of diffusion-controlled biorecognition reactions, magnetic properties can be exploited to improve reaction rates by active mixing. ${ }^{60}$ Furthermore, micromagnetic detection in LOC platforms might be of value for investigations of the native magnetism exhibited, for example, by magnetotactic bacteria, ${ }^{61,62}$ or by magnetic nanoparticles themselves.

\section{Overview of magnetism and magnetic particles Magnetism}

Magnetism is a collective name for relativistic phenomena arising from the movement of an electrical charge. Most commonly, this definition applies to two cases of the movement of the electrons in solid-state matter: 1) the quantum-mechanical orbital and spin motion at the atomic scale, and 2) electric current flow through a conductor. These two cases cover the magnetic phenomena relevant to the research presented in this dissertation. The original definition also spans, for example, the motion of ions and electrons in plasma, ions in a liquid, or quantum charge-density fluctuations within electrically neutral particles (neutrons).

The fundamental manifestation of magnetism is the magnetic force observed as mutual attraction or repulsion of certain materials, and the space in which this force can be observed is referred to as the magnetic field. Since the origin of magnetism lays in the movement of a charge, the magnitude of the magnetic field strength $\mathbf{H}$ is expressed in SI units in $\mathrm{A} \cdot \mathrm{m}^{-1}$. The response of any given material or free space to the externally applied magnetic field is 
defined as magnetic induction $\mathbf{B}$, and is measured in SI units of tesla (T). The relationship between the applied field and the induction is defined as the permeability of the material, $\mu$ :

$$
\mathbf{B}=\mu \mathbf{H}
$$

The permeability of free space is a constant $\mu_{0}=4 \pi \times 10^{-7} \mathrm{~Wb} \cdot \mathrm{A}^{-1} \cdot \mathrm{m}^{-1}, 4 \pi \times 10^{-7} \mathrm{~V} \cdot \mathrm{s} \cdot \mathrm{A}^{-1} \cdot \mathrm{m}^{-}$ ${ }^{1}$, or $4 \pi \times 10^{-7} \mathrm{~kg} \cdot \mathrm{m} \cdot \mathrm{s}^{-2} \cdot \mathrm{A}^{-2}$. However, the permeability of any other medium will generally vary with the applied field. The ratio $\mu_{r}=\mu / \mu_{0}$ is called the relative permeability.

The magnetic moment $\mathbf{m}$ of a magnetic dipole is a vector relating the torque $\tau$ acting on the dipole in a field of the induction $\mathbf{B}$ :

$$
\tau=\mathbf{m} \times \mathbf{B}
$$

Alternatively, an equivalent definition can be based on a current loop represented by the area vector $\mathbf{A}$, and carrying the current $I$ :

$$
\mathbf{m}=I \mathbf{A}
$$

In order to relate the magnetic properties of a material to the magnetic induction, the magnetization $\mathbf{M}$, due to any individual electronic or atomic moments $\mathbf{m}$ existing in the sample, can be defined as the magnetic moment per unit volume:

$$
\mathbf{M}=\frac{1}{V} \sum_{V} \mathbf{m} .
$$

Equation 1.1 can now be rephrased in terms of contributions to the induction from the free-space induction, and from the magnetization of the material occupying that free space:

$$
\mathbf{B}=\mu_{0}(\mathbf{H}+\mathbf{M})
$$

Finally, the magnetic susceptibility $\chi$ of a material can be defined as

$$
\chi=\frac{M}{H}
$$


Although all materials exhibit magnetic properties to some extent, these properties can be more or less pronounced, as dictated by the atomic structure, temperature and pressure. Based on the definitions presented above and the characteristic values of susceptibilities, all materials can be conveniently classified into several major groups: ferromagnetic, antiferromagnetic, ferrimagnetic, diamagnetic, paramagnetic, and superparamagnetic. Ferromagnetism, antiferromagnetism, and ferrimagnetism are related to ordered states. Diamagnetism, paramagnetism, and superparamagnetism exist only as transient states, and are the result of an applied magnetic field.

\section{Ferromagnetism}

In ferromagnetic materials, atomic moments align parallel to each other - due to the exchange interaction - forming magnetic domains. As the exchange interaction decays with the distance, the moments of the adjacent domains will tend to align in opposite directions, as required by the classical case of two dipoles. In the absence of an external field, the bulk magnetization of a ferromagnetic material can therefore be zero. When an external field is applied, the magnetic moments of the domains will start reorienting themselves parallel to the applied field. Once the applied field is strong enough, the material will reach its maximum magnetization, referred to as the saturation magnetization, $\mathbf{M}_{\mathbf{s}}$. At this point, the magnetic induction of the material will continue to rise linearly, as the contribution from the magnetization term will be constant (refer to Eq. 1.5). The domains will stay in a more or less ordered state even when the external field value is brought back to zero, therefore determining what is called the remnant magnetization, $\mathbf{M}_{\mathbf{r}}$. Although not a minimal-energy state, this state of aligned domains may be extremely stable, and is believed to persist for long periods of geologic time. ${ }^{63}$ However, the net magnetization can be destroyed by heating and then cooling (annealing) the material in the absence of an external field. The temperature at which a ferromagnetic material loses its remnant magnetization is known as the Curie point.

The reverse field that is required to reduce the magnetization from $M_{r}$ back to zero is called the intrinsic coercivity, $\mathrm{H}_{\mathrm{ci}}$. This value is related, but not identical, to coercivity $\mathrm{H}_{\mathrm{c}}$, which is defined as the reverse field required to reduce the magnetic induction within the material to zero. The above discussion is illustrated in Figure 5. Magnetic materials with low coercivities are often labeled as soft. On the other hand, magnetically hard materials are those with high coercivities. It is important to keep in mind that when a magnetized ferromagnetic object is free to move, it might be energetically more favorable for the object to rotate against viscous, frictional, and inertial forces, than to rearrange the domain 
magnetizations, when subjected to a reverse field. A familiar example of such behavior would be a compass needle.
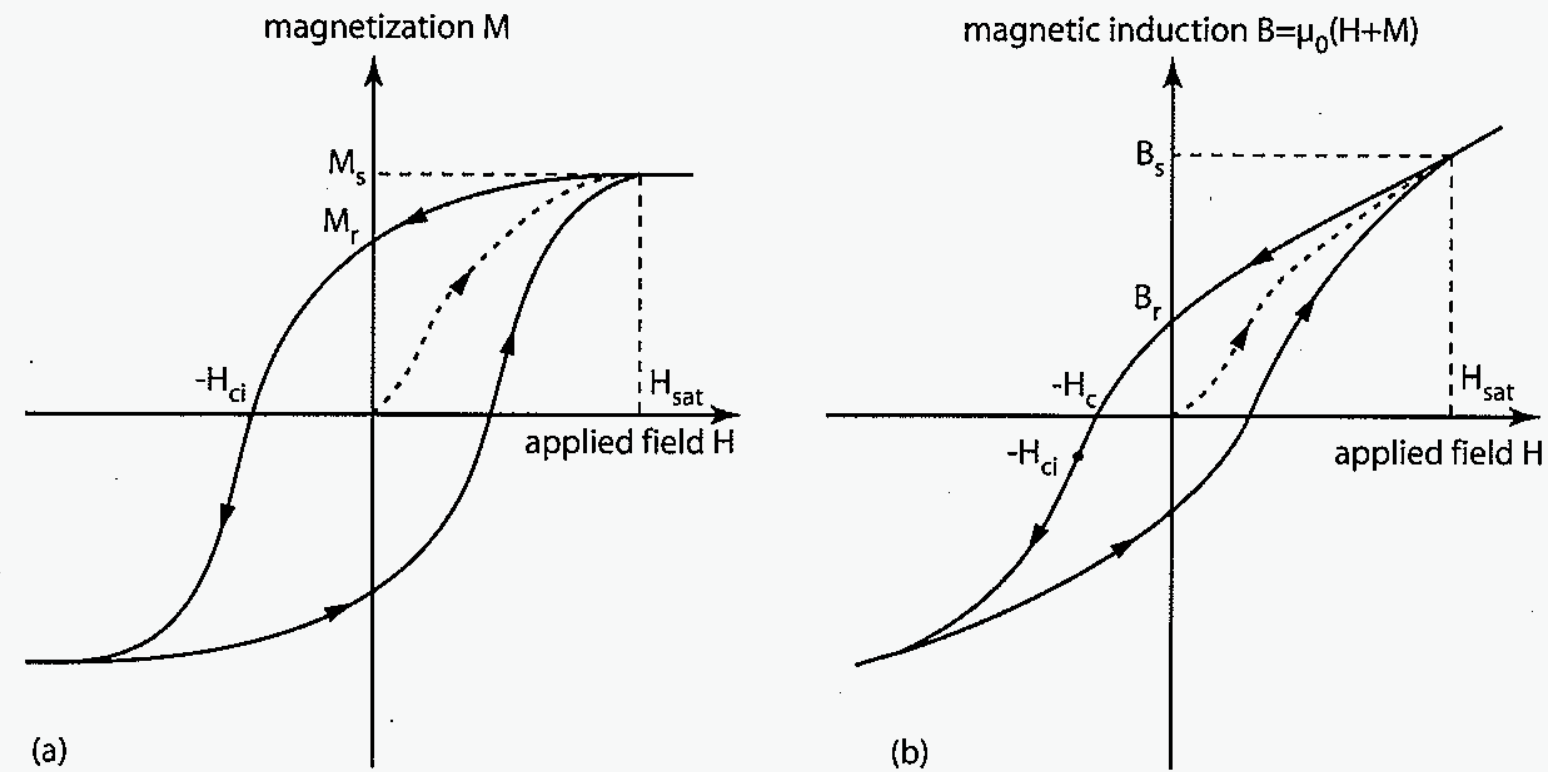

Figure 5. Hysteresis curves of a ferromagnetic material given as magnetization vs. applied field (a), and induction vs. applied field (b). ${ }^{64}$

\section{Antiferromagnetism}

The atomic coupling in antiferromagnetic materials is anti-parallel, and thus the net magnetic moment is zero. The susceptibility is positive and very small. The point at which the antiferromagnetic coupling is destroyed is known as the Néel temperature. Antiferromagnetic materials are relatively uncommon - examples include chromium, ironmanganese alloy (FeMn), and nickel oxide (NiO).

\section{Ferrimagnetism}

Ferrimagnetic materials contain two sublattices of opposite magnetization directions, and are therefore similar to antiferromagnets. The magnetic moments of the coupled atoms, however, are unequal in magnitude; therefore, ferrimagnets can have a net overall magnetization, just like ferromagnets. The oldest known magnetic substance, magnetite $\left(\mathrm{Fe}_{3} \mathrm{O}_{4}\right)$, is a ferrimagnet due to the presence of iron ions in two different oxidation states. Since the moments of two sublattices are anti-parallel, the temperature at which the ferrimagnet ceases to have a permanent moment is called Néel temperature in analogy with antiferromagnets. 


\section{Diamagnetism}

Diamagnetism is only exhibited in the presence of an external field, and results in the induced magnetic moment that is very small and opposite to the applied field. Diamagnetism can be explained as a manifestation of Lenz's law, which states that induced current always flows in the direction as to oppose the change causing it. All materials show a diamagnetic response - however, diamagnetism is often masked by other forms of magnetism. Examples of materials in which diamagnetism is observed include water, DNA, most plastic polymers, gold, and bismuth. Susceptibilities are very small and negative.

\section{Paramagnetism}

Paramagnetism is exhibited when individual atoms have permanent moments, but these moments do not interact. In such case, the atomic moments will align themselves with the applied field, but upon the removal of the field, the thermal motion will quickly randomize the orientation. The susceptibility is positive, and typically less then 0.1 . Paramagnetism is observed, for example, in aluminum, oxygen, platinum, and uranium, but also in ferromagnets above the Curie temperature, and in antiferromagnets and ferrimagnets above the Néel temperature.

\section{Superparamagnetism}

Normally, coupling forces in ferro- or ferrimagnetic materials cause the magnetic moments of neighboring atoms to align, resulting in formation of domains with large internal magnetic fields. At temperatures above the Curie or Neel point, the thermal energy is sufficient to overcome the coupling forces, causing the atomic magnetic moments to fluctuate randomly. The internal magnetic field no longer exists and the material exhibits paramagnetic behavior.

Superparamagnetism occurs when the material is composed of very small, subdomainsized crystallites - typically on the order of nanometers. In this case, even though the thermal energy is not sufficient to overcome the coupling forces between neighboring atoms, it is sufficient to change the direction of magnetization of the entire crystallite. The resulting fluctuations in the direction of magnetization cause the magnetic field to average to zero. The material behaves like a paramagnetic substance. However, instead of each individual atom being independently influenced by an external magnetic field, the magnetic moment of the entire crystallite tends to align with the magnetic field. Susceptibilities of superparamagnets can be significantly higher than those of paramagnetic materials. Magnetization is achieved through a combination of two distinct mechanisms, with different characteristic relaxation 
times, depending on the size of the crystallite. The extrinsic, or Brownian mechanism involves the rotation of the nanoparticle as a whole, including the magnetic moment. The Brownian relaxation time $\tau_{B}$ depends on the hydrodynamic volume $V_{h}$, temperature $T$, and dynamic viscosity $\eta$ of the carrier liquid:

$$
\tau_{B}=\frac{3 V_{h} \eta}{k_{B} T}
$$

where $k_{B}$ is the Boltzmann constant. In the case of a frozen carrier liquid or a solid carrier matrix, $\tau_{B}$ will converge to infinity.

The intrinsic or Néel relaxation mechanism involves the particle moment rotation within the particle. An energy barrier must be overcome for this rotation to happen, and the characteristic time $\tau_{N}$ is described by an exponential function of the particle geometrical volume $V$, and the material-dependent anisotropy constant $K$ (for magnetite, $K=11 \mathrm{~kJ} \cdot \mathrm{m}^{-3}$ ):

$$
\tau_{N}=\tau_{0} \exp \left(\frac{K V}{k_{B} T}\right)
$$

where $\tau_{0} \approx 1 \mathrm{~ns}$. For a 10 -nm magnetite particle at $294 \mathrm{~K}, \tau_{N}=4.1 \mathrm{~ns}$; a 20 -nm particle, however, yields $\tau_{N}$ of $8.5 \mu \mathrm{s}$. In practice; the size and structure of superparamagnetic particles are such that Néel mechanism dominates (i.e., $\tau_{N}$ is much shorter than $\tau_{B}$ ). The magnetization curve of a collection of intrinsically superparamagnetic particles, whether freely rotating or in a frozen carrier, ideally shows no hysteresis - that is, the coercivity equals zero.

\section{Structure and applications of magnetic particles}

There is more than magnetism to magnetic particles. Besides the magnetic material itself, they usually incorporate other functional structures, such as fluorophores, surfactants, biomolecules, or ionic or chemically reactive moieties. Magnetism is then just one of the traits that together determine the synergic functionality needed for a particular application.

Magnetic nanoparticles are commonly defined as magnetic objects with one dimension less than $200 \mathrm{~nm}$. A special case within this category are high aspect ratio structures such as ferromagnetic nanowires. ${ }^{65}$ Another category includes magnetic microspheres or beads, micron-sized polymeric spheres in which magnetic nanoparticles are uniformly dispersed, or grouped to form a magnetic core or shell. Finally, many microorganisms synthesize magnetic 
materials to form intracellular chains of nanoparticles known as magnetosomes. ${ }^{61,62}$ This diverse group of microorganisms is referred to as magnetotactic bacteria. Some reports also suggest the presence of biogenic magnetic particles in animals, possibly as part of the magnetic sensory system. ${ }^{66,67}$

\section{Non-biological applications}

Ferrofluids are colloidal suspensions of superparamagnetic particles in a carrier liquid, ${ }^{68,69}$ and here we refer only to non-biological applications of such systems. Ferrofluid particles are coated with a molecular layer of surfactant, ensuring colloidal stability even in the presence of saturating magnetic fields. Because of the strong particle-carrier interaction imposed by the presence of the surfactant, ferrofluids act as liquid magnets, with magnetization parameters coupled to the usual degrees of freedom of the bulk fluid - for example, viscosity, surface energy, and density. Developed originally by NASA for use as vacuum seals around drive shafts, ferrofluids are nowadays also used in loudspeakers, active vibration damping systems, and as magnetic ink.

Magnetic data storage technologies are benefiting from the advances in nanoparticle research. One of the main obstacles to further increasing density of information in hard drives is the size of individual magnetic grains in thin films used, and problems related to the thermal stability and cross-talk of the downsized grains. ${ }^{70}$ Arrays of highly uniform ferromagnetic ( $\mathrm{FePt}$ or $\mathrm{Co}$, for example) nanoparticles are being investigated as candidates for the next-generation data storage media. ${ }^{71-73}$

Magnetic particle inspection is an industrial process based on using micron-sized magnetic particles to locate structural changes such as fatigue cracks in parts fabricated from magnetizable materials. A typical protocol involves application of the thin film of particle suspension onto the part, and application of the magnetic field. Defects in the material tend to create magnetic field gradients, which attract magnetic particles. To aid visual inspection, particles are often fluorescently labeled.

\section{Biomedical and biotechnological applications}

Magnetic separations of biomolecules or bioparticles are widely practiced today. ${ }^{74,75}$ The magnetic separation process in its basic form involves magnetic particles coupled to a biorecognition agent, such as an antibody or an oligonucleotide, and a magnetic separator unit that retains the particles, allowing for preconcentration, washing, or chemical processing of bound targets. 
Nanoparticles in the form of dextran-coated magnetic clusters, 20-100 nm in size, were introduced by Miltenyi Biotec. ${ }^{75,76}$ The clusters are comprised of iron oxide particles roughly $10 \mathrm{~nm}$ in size (see Figure 5). Another example of functional nanomagnets involves chargebased separations of proteins using lipid-coated magnetite particles. ${ }^{77}$ In most cases of nanometer-sized magnets, induced magnetic moments of individual particles are low enough to require specially designed separators. Upon binding to cells or molecules of interest, separation is achieved by flowing the sample through a column packed with steel wool or millimeter-sized steel spheres in the presence of a magnetic field. High gradients formed in the vicinity of the column packing material attract labeled cells, and retain them if fluidic shear forces are not too strong.

Alternative cell separation methods use larger, micrometer-sized polymeric beads doped with superparamagnetic crystallites. Due to the higher total magnetic content per each bead, the retention can be achieved by simply placing a permanent magnet next to the vial containing the suspension. An even faster separation is obtained by arranging external magnets in a quadrupole configuration, as this provides higher field gradients. ${ }^{78}$ When separating cells, the use of beads has several disadvantages compared to nanoparticles - the bead binding reaction is slower and less efficient due to much lower diffusion coefficients and possible steric hindrance. Furthermore, aggregation of cells and particles can lead to nonspecific trapping of undesired cells, and poses problems in downstream processing through flow cytometry or culturing. Nevertheless, magnetic microspheres are widely used as part of enrichment and separation procedures in microbiology, ${ }^{79}$ immunoassay diagnostics, ${ }^{80}$ and genetic analysis. ${ }^{81,82}$ All separation methods mentioned so far were batch-based however, magnetic labeling also enables for continuous deflection of particles in flow - a principle used to devise a magnetic cell sorter described by Zborowski et al. ${ }^{83}$ and a free-flow magnetophoretic separator described by Pamme et al. ${ }^{17}$

Single-cell biophysical and biochemical analysis arenas have also profited from the use of magnetic particles. Based on the magnetophoretic mobilities derived from optical measurements of labeled cells, it is possible to deduce the cell-antibody binding capacity, and also the kinetics of antibody binding. ${ }^{84,85}$ Ferromagnetic beads can be bound to specific sites on the cell surface, and magnetically manipulated to probe the rheological properties of the cell, and receptor-cytoskeletal interactions in the method known as magnetic twisting cytometry. ${ }^{86,87}$

Magnetic resonance imaging (MRI) contrast agents based on nanoparticles have been developed recently. The contrast in MRI emerges from distributions of proton densities and magnetic relaxation times in biological tissues. Functionalized, target-specific 
superparamagnetic particles can dramatically enhance the MRI contrast by altering the local proton signal decay rates. ${ }^{88,89}$

Hyperthermia, or controlled heating of tissues to temperatures in the $42-46^{\circ} \mathrm{C}$ range, is a promising approach in cancer therapy. In magnetic particle hyperthermia, the heat can be

induced in the particle-laden target tissue by applying an ac magnetic field, ${ }^{88,89}$ thereby greatly diminishing the damage done to the surrounding, healthy tissue. In case of ferro- or ferrimagnetic nanoparticles, the heat generation is related to the hysteresis loss in the oscillating field. Superparamagnetic particles can also generate heat in ac fields. The dissipation results from the orientational relaxation of the particles in a viscous medium.

Drug targeting is a process whereby a cytotoxic drug is attached to magnetic particles, and introduced into the blood stream. External, high-gradient magnetic fields can then be used to concentrate particles at a specific target site, where drug can be released via enzymatic activity, photolysis, or changes in local $\mathrm{pH}^{88,89}$

\section{Overview of giant magnetoresistance technologies}

Magnetoresistance refers to the change in electrical resistance of a material in response to a magnetic field. For most materials, the effect is miniscule. Certain magnetic materials - for example permalloy $\left(\mathrm{Ni}_{81} \mathrm{Fe}_{19}\right)$ - are known to exhibit noticeable magnetoresistance, known as anisotropic magnetoresistance (AMR). ${ }^{90}$ The fundamental reason for this effect is the anisotropic scattering cross-section for conducting electrons, depending on their travel direction relative to the magnetization. This process is a distinct phenomenon from the Hall effect, which stems from the Lorenz force work on moving electrons. The resistance change obtained with AMR materials is usually in the range of several percent.

It was discovered in 1987 and 1988 by two research groups that nanostructures of alternating ferromagnetic and non-ferromagnetic layers exhibit magnetoresistance. ${ }^{91-93}$ For certain thicknesses of the nonmagnetic spacers, the magnetizations of adjacent magnetic layers are oriented antiparallel to each other by antiferromagnetic exchange coupling. ${ }^{94}$ The resistance of the multilayer architecture decreases drastically when the magnetizations progressively align under the applied field, as shown in Figure6. The reported relative change in resistance was up to $80 \%$ at $4.2 \mathrm{~K}$, and $20 \%$ at room temperature, and the observed effect was immediately labeled as "giant magnetoresistance". 

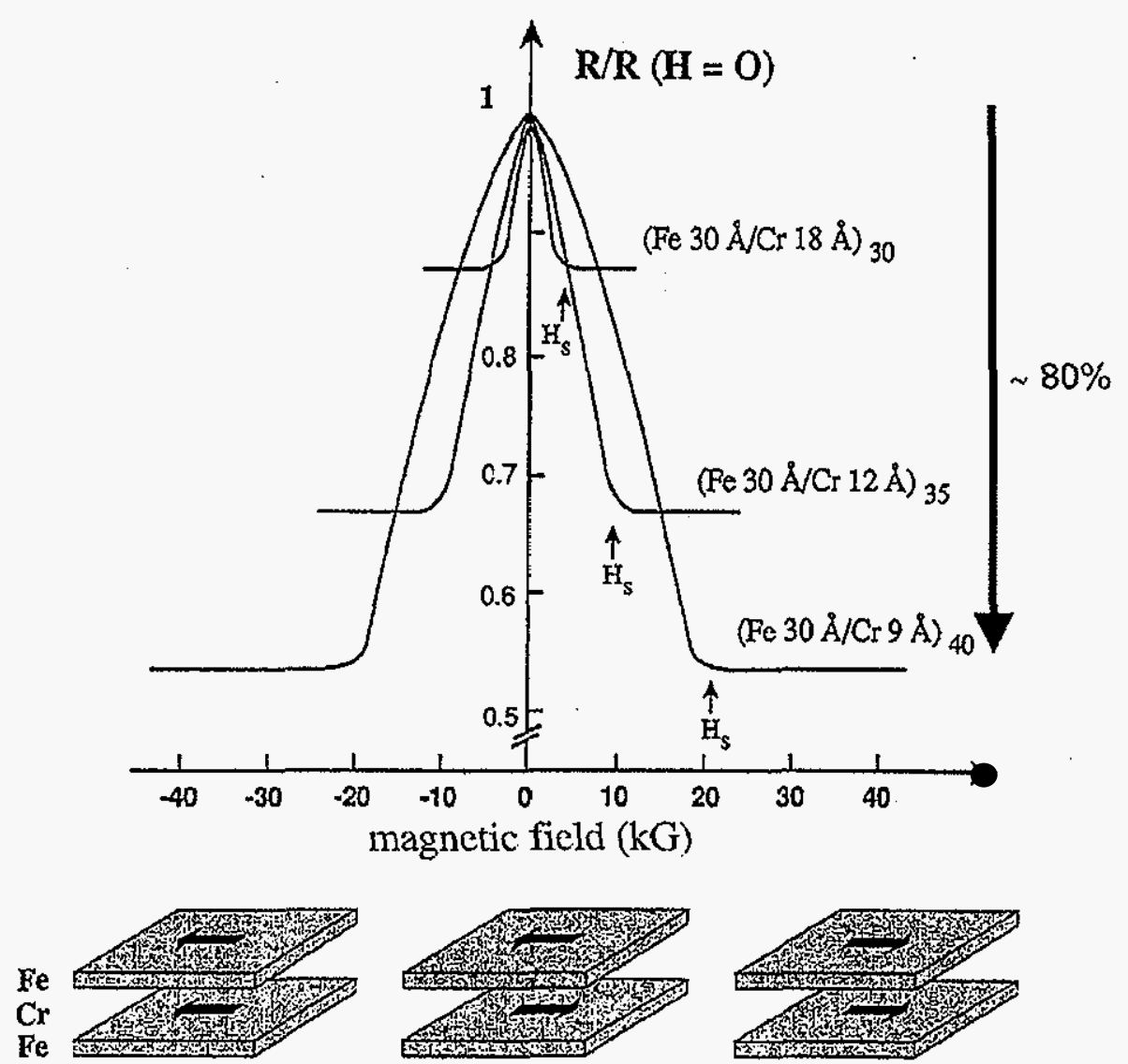

Figure6. Magnetoresistance curves of $\mathrm{Fe} / \mathrm{Cr}$ multilayers at $4.2 \mathrm{~K} .^{92}$

The first commercially available GMR field sensors appeared in 1994, and GMR read heads in hard drives started replacing inductive read heads in 1997, allowing for dramatically higher data densities and improved reliability. ${ }^{93,94}$ In the early 1990s, spin valve GMR structures were introduced, ${ }^{95-98}$ further improving the sensor performance in data storage and low-field sensing applications. In only ten years, magnetic data storage density has increased by two orders of magnitude. ${ }^{99}$ The spin valve multilayer in its basic form is composed of a soft (low coercivity) magnetic layer separated by a nonmagnetic layer from a hard magnetic layer, whose magnetization is pinned through the exchange interaction with an adjacent antiferromagnetic layer (see Figure 7). The most favorable performance is achieved with elongated spin valves in which the fixed layer magnetization is pinned in the transverse direction. ${ }^{44}$ The shape anisotropy will then align the free layer magnetization in the longitudinal direction (easy axis of the free layer). Current can be sourced through the sensor in two geometries: perpendicular to the plane (CPP), or in plane (CIP). The CPP GMR effect 
is typically larger; however, the resistivity for this current flow pathway is usually too low, and measurements in the CIP configurations are currently more practical. $93,94,100$

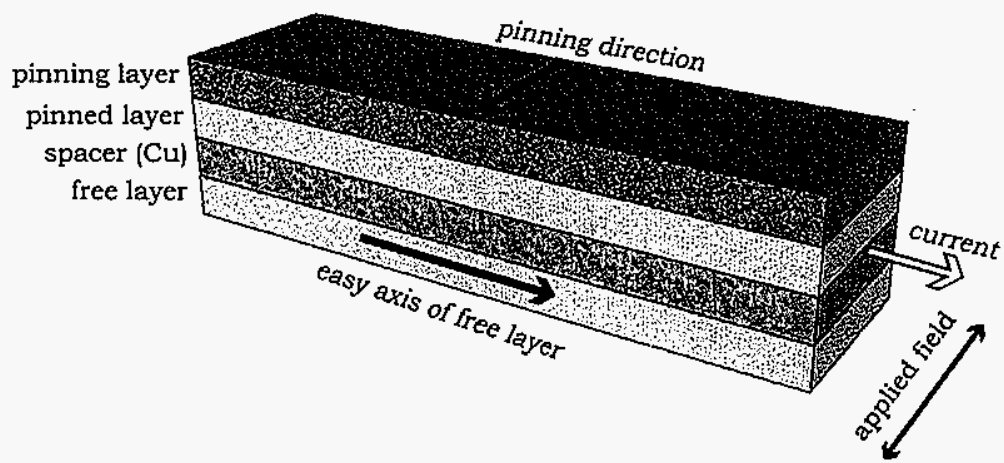

Figure 7. A schematic diagram of the basic spin valve architecture, showing ferromagnetic free and pinned layers separated by a nonmagnetic spacer, and an antiferromagnetic pinning layer. For reasons of clarity, the diagram is not to scale: the thicknesses of ferromagnetic and spacer layers is typically in the nanometer range, while thickness of the pinning layer is on the order of tens of nanometers.

The sensor responds to the field component that is in the sensor plane, and perpendicular to the long axis of the sensor. In a magnetic field, magnetization of the soft layer rotates freely in the response to the field, while the magnetization of the pinned layer remains fixed. At any given angle $\theta$ between the two magnetizations, the resistance $\mathrm{R}$ is given as

$$
R=R_{P}\left(1-A \sin ^{2}\left(\frac{\theta}{2}\right)\right), A=\frac{R_{A P}-R_{P}}{R_{P}},
$$

where $A$ is the GMR value, and $R_{P}$ and $R_{A P}$ are the resistances when the two magnetizations are parallel $\left(\theta=0^{\circ}\right)$ and antiparallel $\left(\theta=180^{\circ}\right)$, respectively. An example of a spin valve sensor response to the applied field is shown in Figure 8. Note that the shape of the transfer function is notably different than that of a multilayer GMR response shown in Figure 6. 


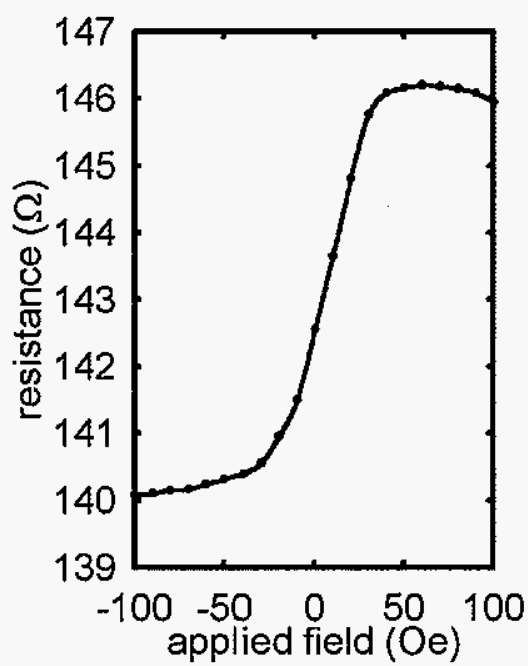

Figure 8. Resistance vs. applied field transfer curve for a spin valve sensor. ${ }^{14}$

The function parity differs, as spin valves exhibit a bipolar response. Next, the useful field range, saturation field, and sensitivity are significantly different. Furthermore, spin valves can be fabricated so that their transfer curve is offset, whereas a multilayer response is normally positioned around the zero field. Each type of discussed magnetoresistive materials - AMR, GMR, spin-valve GMR, as well as the emerging magnetoresistive materials based on tunneling, ${ }^{93}$ has different underlying physics. Some of the parameters to be considered when choosing the sensor appropriate for a given application are: the maximum obtainable change in resistance ( $A$ in Equation 1.9), the saturation field $H_{s}$, sensitivity, thermal stability, noise, and manufacturability. For work presented in the following chapters, we will use spinvalve type GMRs manufactured by NVE Corporation. ${ }^{97}$

This dissertation will detail advances in our laboratory to develop the micromagnetic toolbox for LOC technologies: magnetic micromanipulation systems and various spin-valve sensors for detection of targets ranging from droplets of magnetic fluids to bacterial cells. One of the most important aspects of this study, which draws heavily on mathematical design-based modeling and simulations, is the successful integration of micromagnetic and microfluidic structures into functional and portable devices.

\section{References cited}

1. Moore, G. E. Cramming more components onto integrated circuits. Electronics 38 (1965).

2. Moore, G. E. in Electron-Beam, X-Ray, EUV, and Ion-Beam Submicrometer

Lithographies for Manufacturing V (ed. Warlaumont, J. M.) 2-17 (SPIE, Santa Clara, CA, USA, 1995). 
3. Thorsen, T., Maerkl, S. J. \& Quake, S. R. Microfluidic Large-Scale Integration. Science (Washington, DC) 298, 580-584 (2002).

4. Terry, S. C. A gas chromatography system fabricated on a silicon wafer using integrated circuit technology. Dept. of Electrical Engineering (Stanford University, Stanford, California, 1975).

5. Terry, S. C., Jerman, J. H. \& Angell, J. B. A gas chromatographic air analyzer fabricated on a silicon wafer. IEEE Transactions on Electron Devices ED-26, 1880-6 (1979).

6. Squires, T. M. \& Quake, S. R. Microfluidics: Fluid physics at the nanoliter scale. Reviews of Modern Physics 77, 977-1026 (2005).

7. Lambertus, G. et al. Design, fabrication, and evaluation of microfabricated columns for gas chromatography. Analytical Chemistry 76, 2629-2637 (2004).

8. Bass, A., Chevalier, C. \& Blades, M. W. A capacitively coupled microplasma (CCmP) formed in a channel in a quartz wafer. Journal of Analytical Atomic Spectrometry 16, 919-921 (2001).

9. Reyes, D. R., Ghanem, M. M., Whitesides, G. M. \& Manz, A. Glow discharge in microfluidic chips for visible analog computing. Lab on a Chip 2, 113-116 (2002).

10. Venzie, J. L. \& Marcus, R. K. Micro-scale analytical plasmas for liquid chromatography detection. Analytical and Bioanalytical Chemistry 381, 96-98 (2005).

11. Vilkner, T., Shivji, A. \& Manz, A. Dry powder injection on chip. Lab on a Chip 5, 140145 (2005).

12. Thorsen, T., Roberts, R. W., Arnold, F. H. \& Quake, S. R. Dynamic Pattern Formation in a Vesicle-Generating Microfluidic Device. Physical Review Letters 86, 4163-4166 (2001).

13. Zheng, B., Roach, L. S. \& Ismagilov, R. F. Screening of protein crystallization conditions on a microfluidic chip using nanoliter-size droplets. Journal of the American Chemical Society 125, 11170-11171 (2003).

14. Pekas, N., Porter, M. D., Tondra, M., Popple, A. \& Jander, A. Giant magnetoresistance monitoring of magnetic picodroplets in an integrated microfluidic system. Applied Physics Letters 85, 4783-4785 (2004).

15. Shestopalov, I., Tice, J. D. \& Ismagilov, R. F. Multi-step synthesis of nanoparticles performed on millisecond time scale in a microfluidic droplet-based system. Lab on $a$ Chip 4, 316-321 (2004).

16. El-Ali, J., Gaudet, S., Guenther, A., Sorger, P. K. \& Jensen, K. F. Cell Stimulus and Lysis in a Microfluidic Device with Segmented Gas-Liquid Flow. Analytical Chemistry 77, 3629-3636 (2005).

17. Pamme, N. \& Manz, A. On-Chip Free-Flow Magnetophoresis: Continuous Flow Separation of Magnetic Particles and Agglomerates. Analytical Chemistry 76, 7250-7256 (2004). 
18. Pekas, N., Granger, M., Tondra, M., Popple, A. \& Porter, M. D. Magnetic particle diverter in an integrated microfluidic format. Journal of Magnetism and Magnetic Materials 293, 584-588 (2005).

19. Sadani, Z., Wacogne, B., Pieralli, C., Roux, C. \& Gharbi, T. Microsystems and microfluidic device for single oocyte transportation and trapping: Toward the automation of in vitro fertilizing. Sensors and Actuators, A: Physical A121, 364-372 (2005).

20. Schulte, T. H., Bardell, R. L. \& Weigl, B. H. Microfluidic technologies in clinical diagnostics. Clinica Chimica Acta 321, 1-10 (2002).

21. Wheeler, A. R. et al. Microfluidic device for single-cell analysis. Analytical Chemistry 75, 3581-3586 (2003).

22. Castellana, E. T., Kataoka, S., Albertorio, F. \& Cremer, P. S. Direct Writing of Metal Nanoparticle Films Inside Sealed Microfluidic Channels. Analytical Chemistry 78, 107112 (2006).

23. Jayashree, R. S. et al. Air-Breathing Laminar Flow-Based Microfluidic Fuel Cell. Journal of the American Chemical Society 127, 16758-16759 (2005).

24. Palencsar, A. \& Scherson, D. A. Single-particle electrode aqueous microbatteries. Electrochemical and Solid-State Letters 8, A622-A626 (2005).

25. Breslauer, D. N., Lee, P. J. \& Lee, L. P. Microfluidics-based systems biology. Molecular BioSystems 2, 97-112 (2006).

26. Takayama, S. et al. Subcellular positioning of small molecules. Nature (London) 411, 1016 (2001).

27. Braschler, T., Johann, R., Heule, M., Metref, L. \& Renaud, P. Gentle cell trapping and release on a microfluidic chip by in situ alginate hydrogel formation. Lab on a Chip $\mathbf{5}$, $553-559$ (2005).

28. Segelke, B. Macromolecular crystallization with microfluidic free-interface diffusion. Expert Review of Proteomics 2, 165-172 (2005).

29. Hirschberg, D. et al. Identification of endothelial proteins by MALDI-MS using a compact disc microfluidic system. Protein Journal 23, 263-271 (2004).

30. Kuschel, M. Lab-on-a-chip technology - applications for life sciences. Pharmaceutical Technology Europe 13, 54,56-58 (2001).

31. Seo, J. \& Lee, L. P. Disposable integrated microfluidics with self-aligned planar microlenses. Sensors and Actuators, B: Chemical B99, 615-622 (2004).

32. Edel, J. B. et al. Thin-film polymer light emitting diodes as integrated excitation sources for microscale capillary electrophoresis. Lab on a Chip 4, 136-140 (2004).

33. Hofmann, O. et al. Thin-film organic photodiodes as integrated detectors for microscale chemiluminescence assays. Sensors and Actuators, B: Chemical B106, 878-884 (2005). 
34. Woolley, A. T., Lao, K., Glazer, A. N. \& Mathies, R. A. Capillary Electrophoresis Chips with Integrated Electrochemical Detection. Analytical Chemistry 70, 684-688 (1998).

35. Kuban, P. \& Hauser, P. C. Application of an external contactless conductivity detector for the analysis of beverages by microchip capillary electrophoresis. Electrophoresis 26, 3169-3178 (2005).

36. Fong, L. E. et al. High-resolution room-temperature sample scanning superconducting quantum interference device microscope configurable for geological and biomagnetic applications. Review of Scientific Instruments 76, 053703/1-053703/9 (2005).

37. Trumbull, J. D., Glasgow, I. K., Beebe, D. J. \& Magin, R. L. Integrating microfabricated fluidic systems and NMR spectroscopy. IEEE Transactions on Bio-Medical Engineering 47, 3-7 (2000).

38. McDonnell, E. E., Han, S., Hilty, C., Pierce, K. L. \& Pines, A. NMR Analysis on Microfluidic Devices by Remote Detection. Analytical Chemistry 77, 8109-8114 (2005).

39. La Borde, R. T., Taff, R. N. \& Pratt, D. M. A lateral flow assay device with improved detection access for immunoassay use. Quantum Design, Inc., USA. (2004).2003US18378, 2004011942.

40. Ejsing, L. et al. Planar Hall effect sensor for magnetic micro- and nanobead detection. Applied Physics Letters 84, 4729-4731 (2004).

41. Rife, J. C. et al. Design and performance of GMR sensors for the detection of magnetic microbeads in biosensors. Sensors and Actuators, A: Physical A107, 209-218 (2003).

42. Graham, D. L. et al. Magnetic field-assisted DNA hybridization and simultaneous detection using micron-sized spin-valve sensors and magnetic nanoparticles. Sensors and Actuators, B: Chemical B107, 936-944 (2005).

43. Millen, R. L., Kawaguchi, T., Granger, M. C., Porter, M. D. \& Tondra, M. Giant Magnetoresistive Sensors and Superparamagnetic Nanoparticles: A Chip-Scale Detection Strategy for Immunosorbent Assays. Analytical Chemistry 77, 6581-6587 (2005).

44. $\mathrm{Li}, \mathrm{G}$. et al. Spin valve sensors for ultrasensitive detection of superparamagnetic nanoparticles for biological applications. Sensors and Actuators, A: Physical A126, 98$106(2006)$.

45. Fulton, R. J., McDade, R. L., Smith, P. L., Kienker, L. J. \& Kettman, J. R., Jr. Advanced multiplexed analysis with the FlowMetrix system. Clinical Chemistry (Washington, $D$. C.) $43,1749-1756$ (1997).

46. Anderson, G. P. et al. TNT Detection Using Multiplexed Liquid Array Displacement Immunoassays. Analytical Chemistry 78, 2279-2285 (2006).

47. Gucker, F. T., Jr., O'Konski, C. T., Pickard, H. B. \& Pitts, J. N., Jr. Photoelectronic counter for colloidal particles. Joumal of the American Chemical Society 69, 2422-31 (1947). 
48. Shapiro, H. M. Microbial analysis at the single-cell level: tasks and techniques. Journal of microbiological methods 42, 3-16 (2000).

49. Göttlinger, C., Mechtold, B. \& Radbruch, A. in Flow Cytometry and Cell Sorting (ed. Radbruch, A.) 3-25 (Springer-Verlag, Berlin, 2000).

50. Raybourne, R. \& Tortorello, M. in Detecting pathogens in food (ed. McMeekin, T. A.) (Woodhead Publishing, Cambridge, England, 2003).

51. Kubitschek, H. E. Electronic counting and sizing of bacteria. Nature (London) 182, 234-5 (1958).

52. Hoffman, R. A. \& Britt, W. B. Flow-system measurement of cell impedance properties. The journal of histochemistry and cytochemistry : official journal of the Histochemistry Society 27, 234-40 (1979).

53. Menke, E., Kordwig, E., Stuhlmuller, P., Kachel, V. \& Ruhenstroth-Bauer, G. A volumeactivated cell sorter. The journal of histochemistry and cytochemistry : official journal of the Histochemistry Society 25, 796-803 (1977).

54. Wang, M. M. et al. Microfluidic sorting of mammalian cells by optical force switching. Nature Biotechnology 23, 83-87 (2005).

55. Lancaster, C. et al. Rare cancer cell analyzer for whole blood applications: Microcytometer cell counting and sorting subcircuits. Methods (San Diego, CA, United States) 37, 120-127 (2005).

56. Valer, M. Measuring multiple apoptosis parameters on a microfluidics chip-based instrument. American Biotechnology Laboratory 21, 28L-28N (2003).

57. Gawad, S., Cheung, K., Seger, U., Bertsch, A. \& Renaud, P. Dielectric spectroscopy in a micromachined flow cytometer: theoretical and practical considerations. Lab on a Chip $\mathbf{4}$, 241-251 (2004).

58. Cheung, K., Gawad, S. \& Renaud, P. Impedance spectroscopy flow cytometry: on-chip label-free cell differentiation. Cytometry, Part A 65A, 124-132 (2005).

59. Chun, H., Chung, T. D. \& Kim, H. C. Cytometry and Velocimetry on a Microfluidic Chip Using Polyelectrolytic Salt Bridges. Analytical Chemistry 77, 2490-2495 (2005).

60. Suzuki, H. \& Ho, C.-M. A magnetic force driven chaotic micro-mixer. IEEE International Conference on Micro Electro Mechanical Systems, Technical Digest, 15th, Las Vegas, NV, United States, Jan. 20-24, 2002, 40-43 (2002).

61. Blakemore, R. Magnetotactic bacteria. Science 190, 377-9 (1975).

62. Bazylinski, D. A. \& Frankel, R. B. Magnetosome formation in prokaryotes. Nature Reviews Microbiology 2, 217-230 (2004).

63. Chang, S. B. R. \& Kirschvink, J. L. Magnetofossils, the magnetization of sediments, and the evolution of magnetite biomineralization. Annual Review of Earth and Planetary Sciences 17, 169-95 (1989). 
64. Sung, H. W. F. \& Rudowicz, C. Physics behind the magnetic hysteresis loop-a survey of misconceptions in magnetism literature. Journal of Magnetism and Magnetic Materials 260, 250-260 (2003).

65. Reich, D. H. et al. Biological applications of multifunctional magnetic nanowires (invited). Journal of Applied Physics 93, 7275-7280 (2003).

66. Diebel, C. E., Proksch, R., Green, C. R., Nellson, P. \& Walker, M. M. Magnetite defines a vertebrate magnetoreceptor. Nature (London) 406, 299-302 (2000).

67. Johnsen, S. \& Lohmann, K. J. The physics and neurobiology of magnetoreception. Nature Reviews Neuroscience 6, 703-712 (2005).

68. Odenbach, S. (ed.) Ferrofluids: Magnetically Controllable Fluids and Their Applications (Springer, Berlin - New York, 2002).

69. Skumiel, A., Jozefczak, A., Hornowski, T. \& Labowski, M. The influence of the concentration of ferroparticles in a ferrofluid on its magnetic and acoustic properties. Journal of Physics D: Applied Physics 36, 3120-3124 (2003).

70. Sellmyer, D. J., Zeng, H., Yan, M., Sun, S. \& Liu, Y. in Handbook of.Advanced Magnetic Materials (eds. Liu, Y., Sellmyer, D. J. \& Shindo, D.) 211-240 (2006).

71. Sun, S., Murray, C. B., Weller, D., Folks, L. \& Moser, A. Monodisperse FePt nanoparticles and ferromagnetic FePt nanocrystal superlattices. Science (Washington, D. C.) $287,1989-1992$ (2000).

72. Eggeman, A. S. et al. Synthesis and characterisation of silica encapsulated cobalt nanoparticles and nanoparticle chains. Journal of Magnetism and Magnetic Materials 301, 336-342 (2006).

73. Weekes, S. M., Ogrin, F. Y. \& Murray, W. A. Fabrication of Large-Area Ferromagnetic Arrays Using Etched Nanosphere Lithography. Langmuir 20, 11208-11212 (2004).

74. Molday, R. S., Yen, S. P. \& Rembaum, A. Application of magnetic microspheres in labelling and separation of cells. Nature (London) 268, 437-8 (1977).

75. Miltenyi, S., Muller, W., Weichel, W. \& Radbruch, A. High gradient magnetic cell separation with MACS. Cytometry 11, 231-8 (1990).

76. Kantor, A. B., Gibbons, I., Miltenyi, S., Schmitz, J. in Cell Separation Methods and Applications (eds. Recktenwald, D. \& Radbruch, A.) (Marcel Dekker, New York, 1998).

77. Bucak, S., Jones, D. A., Laibinis, P. E. \& Hatton, T. A. Protein Separations Using Colloidal Magnetic Nanoparticles. Biotechnology Progress 19, 477-484 (2003).

78. Hatch, G. P. \& Stelter, R. E. Magnetic design considerations for devices and particles used for biological high-gradient magnetic separation (HGMS) systems. Journal of Magnetism and Magnetic Materials 225, 262-276 (2001). 
79. Hu, J. et al. Improvement of recoveries for the determination of protozoa Cryptosporidium and Giardia in water using method 1623. Journal of Microbiological Methods 58, 321-325 (2004).

80. Hayes, M. A., Polson, N. A., Phayre, A. N. \& Garcia, A. A. Flow-based microimmunoassay. Analytical Chemistry 73, 5896-5902 (2001).

81. Degenkolbe, T. et al. A quality-controlled microarray method for gene expression profiling. Analytical Biochemistry 346, 217-224 (2005).

82. Smistrup, K. et al. On-chip magnetic bead microarray using hydrodynamic focusing in a passive magnetic separator. Lab on a Chip 5, 1315-1319 (2005).

83. Zborowski, M., Sun, L., Moore, L. R., Williams, P. S. \& Chalmers, J. J. Continuous cell separation using novel magnetic quadrupole flow sorter. Joumal of Magnetism and Magnetic Materials 194, 224-230 (1999).

84. Moore, L. R. et al. The use of magnetite-doped polymeric microspheres in calibrating cell tracking velocimetry. Journal of Biochemical and Biophysical Methods 44, 115-130 (2000).

85. Leigh, D. R., Steinert, S., Moore, L. R., Chalmers, J. J. \& Zborowski, M. Cell tracking velocimetry as a tool for defining saturation binding of magnetically conjugated antibodies. Cytometry, Part A 66A, 103-108 (2005).

86. Butler, J. P. \& Kelly, S. M. A model for cytoplasmic rheology consistent with magnetic twisting cytometry. Biorheology 35, 193-209 (1998).

87. Moller, W., Nemoto, I. \& Heyder, J. Effect of magnetic bead agglomeration on Cytomagnetometric measurements. IEEE Transactions on Nanobioscience 2, 247-54 (2003).

88. Pankhurst, Q. A., Connolly, J., Jones, S. K. \& Dobson, J. Applications of magnetic nanoparticles in biomedicine. Journal of Physics D: Applied Physics 36, R167-R181 (2003).

89. Ito, A., Shinkai, M., Honda, H. \& Kobayashi, T. Medical application of functionalized magnetic nanoparticles. Journal of Bioscience and Bioengineering 100, 1-11 (2005).

90. McGuire, T. R. \& Potter, R. I. Anisotropic magnetoresistance in ferromagnetic 3d alloys. IEEE Transactions on Magnetics MAG11, 1018-38 (1975).

91. Sato, H., Schroeder, P. A., Slaughter, J., Pratt, W. P., Jr. \& Abdul-Razzaq, W. Galvanomagnetic properties of silver/M (M = iron, nickel, cobalt) layered metallic films. Superlattices and Microstructures 4, 45-50 (1988).

92. Baibich, $M$. N. et al. Giant magnetoresistance of (001)iron/(001)chromium magnetic superlattices. Physical Review Letters 61, 2472-5 (1988).

93. Wang, D. in Handbook of Advanced Magnetic Materials (eds. Liu, Y., Sellmyer, D. J. \& Shindo, D.) $307-338$ (2006). 
94. Barthelemy, A. et al. Magnetoresistance and spin electronics. Journal of Magnetism and Magnetic Materials 242-245, 68-76 (2002).

95. Dieny, B. et al. Spin-valve effect in soft ferromagnetic sandwiches. Journal of Magnetism and Magnetic Materials 93, 101-4 (1991).

96. Anthony, T. C., Brug, J. A. \& Zhang, S. Magnetoresistance of symmetric spin valve structures. IEEE Transactions on Magnetics 30, 3819-21 (1994).

97. Qian, Z., Daughton, J. M., Wang, D. \& Tondra, M. Magnetic design and fabrication of linear spin-valve sensors. IEEE Transactions on Magnetics 39, 3322-3324 (2003).

98. Freitas, P. P. et al. Spin valve sensors. Sensors and Actuators, A: Physical A81, 2-8 (2000).

99. Keener, C. D. in Handbook of Advanced Magnetic Materials (eds. Liu, Y., Sellmyer, D. J. \& Shindo, D.) 271-291 (2006).

100. Prinz, G. A. Review: device physics. Magnetoelectronics. Science (Washington, D. C.) 282, 1660-1663 (1998). 


\title{
CHAPTER 2. MAGNETIC PARTICLE DIVERTER IN AN INTEGRATED MICROFLUIDIC FORMAT
}

\author{
A paper published in Journal of Magnetism and Magnetic Materials ${ }^{*}$ \\ Nikola Pekas, ${ }^{\text {a) }}$ Michael Granger, ${ }^{\text {a) }}$ Mark Tondra, ${ }^{\text {b) }}$ Anthony Popple, ${ }^{\text {b) }}$ and Marc D. \\ Porter $^{\text {a) }}$ \\ a) Institute for Combinatorial Discovery, Departments of Chemistry and Chemical \\ Engineering, and Ames Laboratory - USDOE; Iowa State University, Ames, Iowa 50011 \\ USA.

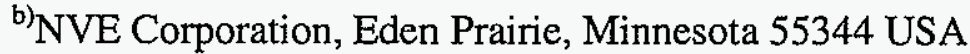

\begin{abstract}
A fully integrated micromagnetic particle diverter and microfluidic system are described. Particles are diverted via an external uniform magnetic field perturbed at the microscale by underlying current straps. The resulting magnetic force deflects particles across a flow stream into one of two channels at a Y-shaped junction. The basic theoretical framework, design, and operational demonstration of the device are presented.
\end{abstract}

\section{Introduction}

Magnetic particles coupled to biorecognition agents, such as antibodies or oligonucleotides, are widely used for cell and organelle separation, DNA and protein isolation and purification, and drug screening [1-4]. In these applications, the particle-target complex is retained by the field of an external permanent magnet in order to separate the target from the sample. Magnetic tagging also potentially provides a means to capture, sort, mix, and direct particles in a variety of lab-on-a-chip devices without the use of mechanical parts, such as valves. To realize this goal, however, it is necessary to generate and dynamically control the magnetic field profiles at a micrometer length scale by using, for example, microfabricated electromagnets.

\footnotetext{
* Reprinted from Journal of Magnetism and Magnetic Materials 293, Magnetic particle diverter in an integrated microfluidic format. Pekas, N., Granger, M., Tondra, M., Popple, A. \& Porter, M. D., 584-588, Copyright 2005, with permission from Elsevier.
} 
Several microelectromagnet-based, particle-actuation strategies have been reported [511]. In some cases, superparamagnetic particles were simply attracted towards a field maximum that was induced by a microelectromagnet in either non-flowing $[5,6]$ or flowing [7,8] suspensions. In previous work [9], we employed a secondary uniform magnetic field to magnetize particles, allowing each microelectromagnetic element to either attract or repel particles, depending on current polarity. Although we demonstrated particle manipulation at the micrometer scale, fluidic integration challenges limited operation to a non-flowing suspension. Other groups exploited a similar approach in order to manipulate particles in non-flowing suspensions at the $100-\mu \mathrm{m} \mathrm{[10]} \mathrm{and} \mathrm{millimeter} \mathrm{[11]} \mathrm{scale.}$

In this report, we describe the first micromagnetic-microfluidic hybrid structure that exerts both attractive and repulsive forces at the micrometer size scale. The design and fabrication, theoretical assessment, and functional demonstration of the rapid and effective diversion of magnetic entities within a fluid stream are presented.

\section{Fabrication}

The magnetic force acting on a particle in a magnetic field is proportional to both the particle magnetic moment and the field gradient. The magnetic moment, in turn, is determined by the magnetic field strength and the intrinsic properties of the particle. In our system, a uniform external magnetic field magnetizes the particles, while microfabricated current lines induce a localized field perturbation. As a result, gradients of $10^{2}-10^{3} \mathrm{~T} / \mathrm{m}$ are generated. Figure 1 provides further details. 

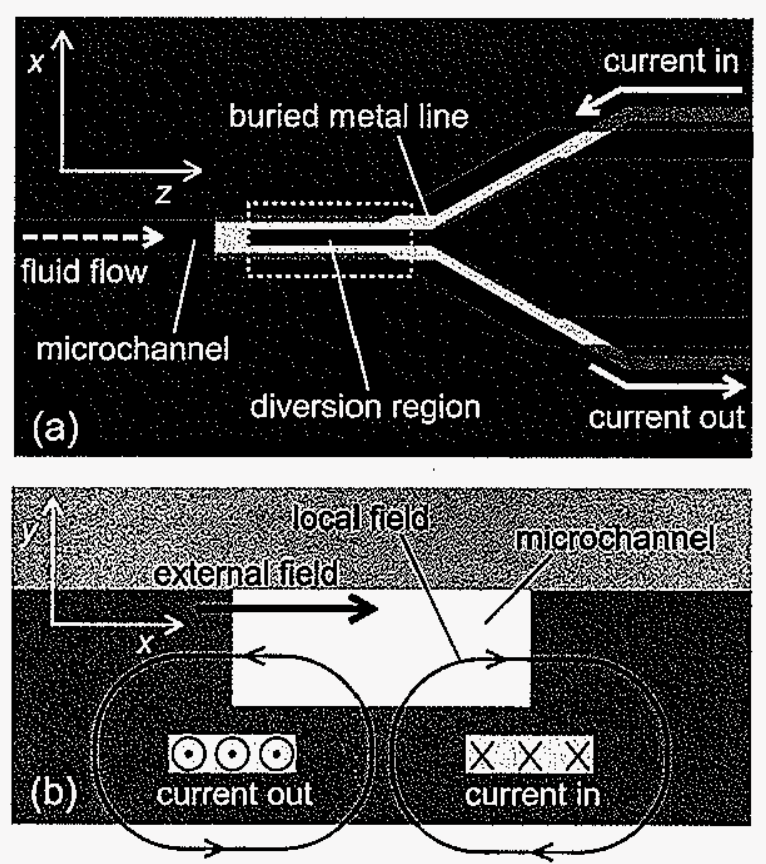

FIG. 1. A micromagnetofluidic diverter: (a) top view and (b) end view schematics. Before the flow-splitting junction, the suspension of magnetic particles flows along the $z$-axis. Magnetic particles in the flow stream are magnetized by an external magnetic field aligned along the $x$-axis. The current-carrying metal straps induce a local gradient field that is superimposed on the external field. The magnetic force, which is proportional to the particle magnetization and to the field gradient, deflects the particles away from the field minimum and toward the field maximum. As a consequence, the particles are driven across the flow stream along the $x$-axis and diverted into a desired channel.

Device fabrication began with the deposition of a $200-\mathrm{nm}$ silicon nitride passivation layer onto a silicon wafer. Next, $1.7-\mu \mathrm{m}$ thick aluminum current lines were sputter-deposited and etched using reactive ion etching (RIE) and a photoresist etch mask. After etching, the structure was coated with a planarizing layer of benzocyclobuthene (BCB), which had a thickness of $1.8 \mu \mathrm{m}$ with respect to the top of the aluminum lines. A second BCB layer was then spun to a $6-\mu \mathrm{m}$ thickness, and etched to form $18-\mu \mathrm{m}$ wide fluidic channels positioned directly over the current lines. The second BCB layer was coated with $200 \mathrm{~nm}$ of silicon nitride to facilitate bonding to a $2-\mathrm{mm}$ thick rectangular slab of poly(dimethyl siloxane) (PDMS) rubber. This step defined the top of the fluidic channels, and added throughchannels for external access to the device. The slabs were made by casting a PDMS prepolymer mixture against a polished silicon wafer. Fluidic connections through the slabs were formed by using sacrificial L-shaped inserts affixed to the wafer prior to molding. After curing the PDMS, the inserts were removed and PDMS slab was peeled off the wafer. Both the slabs and silicon nitride-coated devices were activated in oxygen plasma and then aligned and brought into contact using a mask aligner to seal the slab to the device. Finally, the 
devices were wire-bonded onto printed-circuit boards and tested for electrical and fluidic integrity.

A finished device, mounted on a plastic holder, is shown in Fig. 2. The external field, oriented along the $x$-axis direction, was applied by placing two miniature rare-earth magnets immediately adjacent to the holder sides. The field uniformity and strength were characterized with the gauss-meter probe that was attached to a micromanipulator. We positioned the magnets relative to the device so that the field magnitude equaled $16.0 \mathrm{mT}$, with the variation of $0.6 \mathrm{mT} / \mathrm{mm}$ along the $z$-axis and $0.3 \mathrm{mT} / \mathrm{mm}$ along the $x$-axis. The expected gradients induced by the current straps were at least three orders of magnitude larger than the measured variation in the external field.

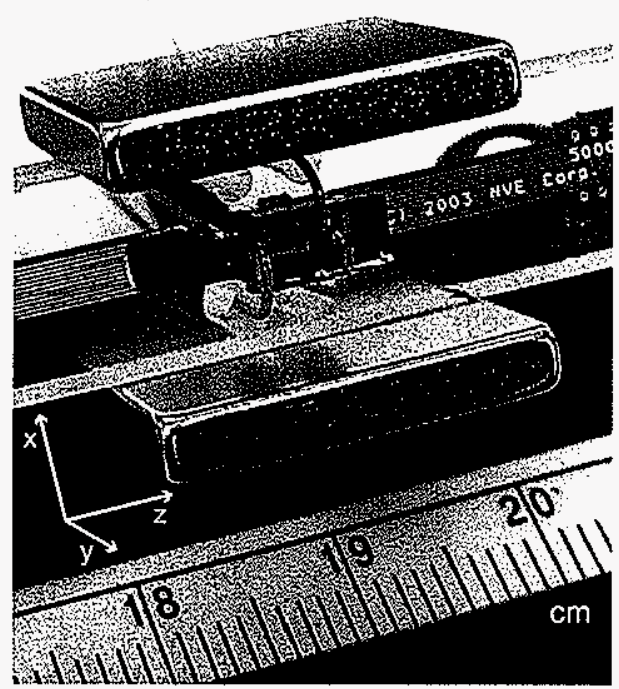

FIG. 2. A micromagnetofluidic device with complete electrical and fluidic connections, mounted on a plastic holder. Fluidic lines (polymeric capillary tubing) are connected to the device through fluidic-access ports located in the sides of the transparent PDMS lid. The device is wire-bonded to a flexible printed-circuit board, and contacts are the potted with a non-conductive epoxy resin. An external magnetic field along the $x$-axis direction is applied by sandwiching the device between the two miniature square-shaped permanent magnets.

\section{Results and Discussion}

To assess performance for a set of experimental parameters, we first modeled the diverter via the following analysis. In a dilute aqueous suspension, the magnetic force $\mathbf{F}_{\text {mag }}$ acting on a superparamagnetic particle in a magnetic field of a free-air inductance $\mathbf{B}$ is given in SI units as $[12,13]$

$$
\mathbf{F}_{m a g}=\frac{V \chi}{\mu_{0}}(\mathbf{B} \cdot \nabla) \mathbf{B},
$$


where $V$ is the particle volume, $\chi$ is the magnetic susceptibility of the particle, and $\mu_{0}$ is the magnetic permeability in vacuum. Since the current density $\mathbf{J}$ within the microchannel equals zero, Ampère's law $\left(\nabla \times \mathbf{B}=\mu_{0} \mathbf{J}\right)$ indicates that the curl of vector $\mathbf{B}$ vanishes within the volume element defined by the microfluidic channel. We can therefore use the identity $\nabla(\mathbf{B} \cdot \mathbf{B})=2 \mathbf{B} \times(\nabla \times \mathbf{B})+2(\mathbf{B} \cdot \nabla) \mathbf{B}$ to transform Eq. 2.1 into:

$$
\mathbf{F}_{m a g}=\frac{V \chi}{2 \mu_{0}} \nabla B^{2}
$$

The magnetic force acting on the particle can now be calculated using the Eq. 2.2. Because of the diverter geometry (long and narrow buried current straps), we can reduce the problem to two dimensions. Thus, the field at any point in the channel cross-section ( $x-y$ plane) can be calculated as a sum of the external field and the current-induced contributions determined from the Biot-Savart law. The modeling results, obtained using in-house code written in MATLABß, are shown in Fig. 3. Although not determined experimentally, an extrapolation of the data provided by the vendor for similar particles yielded a $\chi$ of about 0.1 used in the calculations. In order for the diverter to work effectively, the direction and magnitude of the magnetically derived force on the particle must be relatively uniform. That is, the width and the position of the channel must be designed relative to the current lines in such a way that particles experience only the field flanked by the two straps.

A particle trajectory in the $x-y$ plane can be deduced from the equation of motion involving a viscous-drag force and a magnetic force:

$$
m \frac{d \mathbf{v}}{d t}=-3 \pi \eta a \mathbf{v}+\mathbf{F}_{m a g}
$$

where $m$ is the mass of a particle, $\mathbf{v}$ is the particle velocity across the flow stream, $a$ is the particle diameter, and $\eta$ is the dynamic viscosity of the carrier fluid ( $\eta=0.890 \mathrm{mPa} \cdot \mathrm{s}$ at room temperature [14]). To examine the behavior of a particle, we will assume that $\mathbf{F}_{\mathrm{mag}}$ is a constant perpendicular to the $z$-axis. The particle will then accelerate along the direction of the force, and the velocity dependence on time is obtained from Eq. 2.3:

$$
v(t)=\frac{F_{\text {mag }}}{3 \pi \eta a}\left[1-\exp \left(\frac{3 \pi \eta a}{m} t\right)\right]
$$



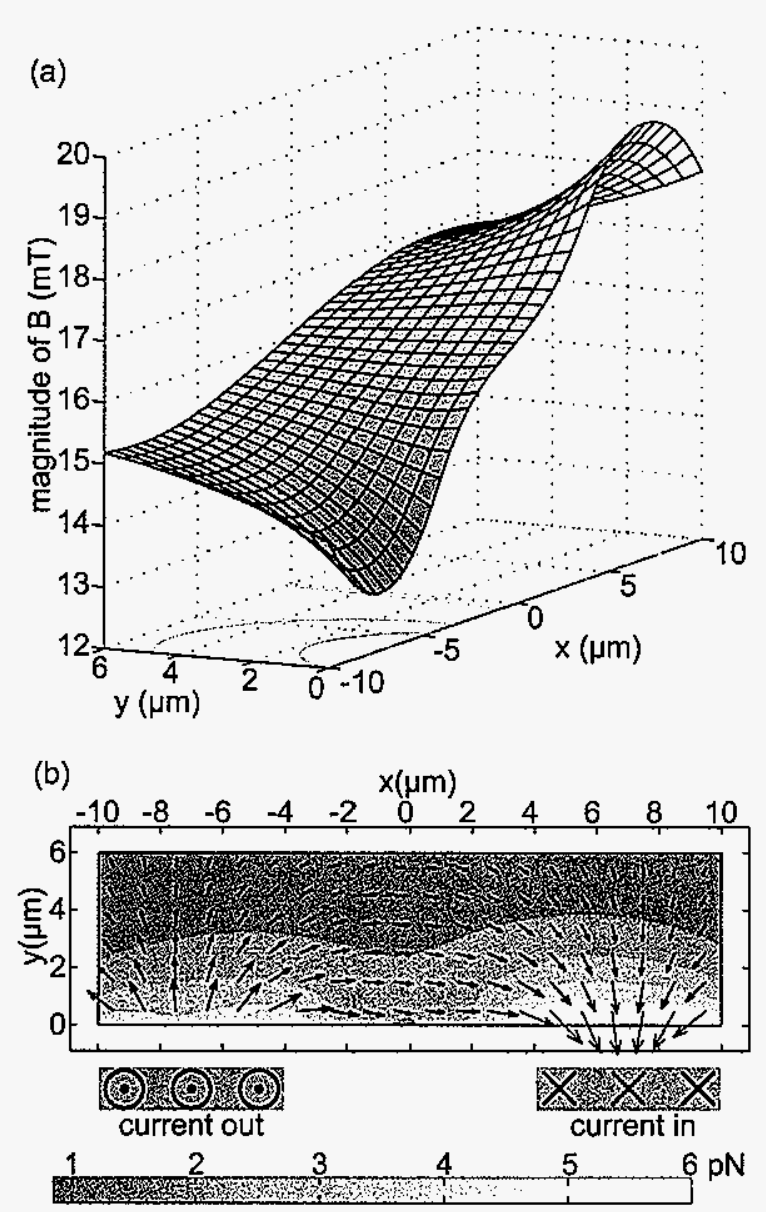

FIG. 3. The magnetic-field and force modeling results, based on a current of $50 \mathrm{~mA}, 16-\mathrm{mT}$ external uniform field applied along the positive $x$-axis direction, a particle susceptibility of 0.1 , and a particle diameter of $1 \mu \mathrm{m}$. (a) The total magnitude of the magnetic field, calculated as a sum of the external field and the current-induced local field. The resultant magnetic force (b), which follows the steepest field gradient.

The results (based on a particle diameter of $1 \mu \mathrm{m}$, and particle density of $1.4 \mathrm{~g} / \mathrm{cm}^{3}$ ) show the following: (1) The diverting force acting on a particle is on the order of several piconewtons. (2) According to the Eq. 2.4, the terminal velocity is on the order of several hundreds of micrometers per second, and the particle reaches $90 \%$ of its terminal across-theflow velocity in only about $180 \mathrm{~ns}$. This result indicates that the particle trajectory will closely follow the force field shown in Fig. 3 at the force and length scale of our system. (3) A particle is diverted to the desired channel when magnetic force drags the particle across the channel for up to one-half of the channel width. Since our present design has a 100- $\mu \mathrm{m}$ long diverter region with $18-\mu \mathrm{m}$ wide channels, the maximum flow velocity is about $3 \mathrm{~mm} / \mathrm{s}$.

To visualize the flow of the fluorescent superparamagnetic beads (Bangs Labs 4962, 28\% magnetite, $0.96 \mu \mathrm{m}$ nominal diameter, internally labeled with the Dragon Green dye), we 
used an epifluorescence microscope equipped with a $\mathrm{CCD}$ camera. A dilute aqueous suspension of magnetic beads $\left(5.3 \times 10^{6}\right.$ beads per $\mathrm{cm}^{3}$, which corresponds to a volume fraction of $2.8 \times 10^{-6}$ ) was pumped from a pressurized vial, with the pumping pressure of 0.1 $0.3 \mathrm{bar}$. The volume flow rate and the consequent bead velocities were held at $\sim 6 \mathrm{~nL} / \mathrm{min}$ and $\sim 1 \mathrm{~mm} / \mathrm{s}$, respectively. A 50-mA current was sourced through the diverter current lines, and images were collected at the rate of one frame per second, with an exposure time of $28 \mathrm{~ms}$ per frame. The collected snapshots were processed using Metamorph software by merging the stack of the snapshots into a "see-through" plane in order to present the data in a single image.

A set of first results is presented in Fig. 4. The beads, originally distributed evenly, were directed into the desired channel after the junction, as dictated by the current polarity. We can define the diversion effectiveness function, $\varepsilon$, as $\varepsilon=(p-q) /(p+q)$, where $p$ and $q$ are the respective numbers of beads detected in the desired and undesired channel after the junction. When all the particles are successfully diverted, $\varepsilon$ equals 1 . When the diverter is inactive, i.e. no current is sourced, $p$ and $q$ will be equal and $\varepsilon$ equals zero. For the data in Fig. $4, \varepsilon$ is found to be 0.71 . This value corresponds to $85 \%$ of the beads flowing down the desired channel, compared to $50 \%$ when the diverter was inactive.
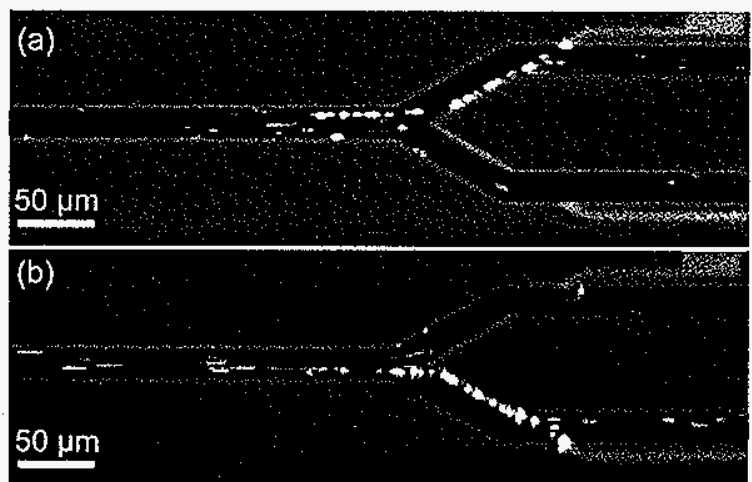

FIG. 4. (a) Fluorescence microphotograph of the diversion region and the flow-splitting junction. The suspension of fluorescent superparamagnetic beads is pumped down the microchannel in the positive $z$-axis direction. The image is a composite "see-through" plane of 60 individual snapshots collected at a $1 \mathrm{~Hz}$ frame rate. Immediately before the flow-splitting junction, particles flow through the $100-\mu \mathrm{m}$ long magnetic-diverter arena (buried current lines not visible) and are deflected to the upper channel. In (b) the current polarity is inversed, and particles are consequently directed into the lower channel.

We attribute the less-than-100\% effectiveness to one or both of two factors. First, the actual channel width was $18 \mu \mathrm{m}$ instead of the targeted $12 \mu \mathrm{m}$ due to an overetched BCB layer. Because of a larger width, the magnetic beads flowing close to one of the walls may experience a portion of the field where the force direction is opposite that of the desired one 
(see Fig. 3). Second, the distribution of the bead sizes was relatively broad, and combining the Eqs. 2.2 and 2.4 shows that the magnetophoretic velocity of a bead scales with the bead cross-section. Although quantitative data was not available, it is likely that a certain fraction of beads were insufficiently large to realize effective diversion.

\section{Conclusions}

In summary, we have demonstrated the valveless, micromagnetic diversion of particles in the flowing stream. Our system is the first reported micromagnetofluidic structure that exerts both attractive and repulsive forces at the micrometer scale. Potential applications are in the area of biomolecule and bioparticle manipulation within microfluidic networks for lab-on-achip combinatorial analysis and synthesis. More complex actuation tasks and massively parallel, integrated design are possible and practical by drawing on microfabrication technology already in place.

This work was supported by grants from the NSF and from the DARPA BioMagnetICs program and by the Institute for Combinatorial Discovery of Iowa State University. The Ames Laboratory is operated for the U.S. Department of Energy by Iowa State University under contract no. W-7405-eng-82.

\section{References cited}

[1] A. B. Kantor, I. Gibbons, S. Miltenyi, and J. Schmitz, in: Cell Separation Methods and Applications, Marcel Dekker, New York, 1998.

[2] M. D. Fisher and S. C. Frost, Anal. Biochem. 251 (1997) 125.

[3] E. Racila, D. Euhus, A. J. Weiss, C. Rao, J. McConnell, L. W. M. M. Terstappen, and J. W. Uhr, Proc. Natl. Acad. Sci. USA 95 (1998), 4589.

[4] G. P. Hatch and R. E. Stelter, J. Magn. Magn. Mater. 225 (2001) 262.

[5] C. S. Lee and R. M. Westervelt, Appl. Phys. Lett. 79 (2001) 3308.

[6] D. L. Graham, H. Ferreira, J. Bernardo, P. P. Freitas, and J. M. S. Cabral, J. Appl. Phys. 91 (2002) 7786.

[7] J.-W. Choi, K. W. Oh, A. Han, et al., Biomed. Microdevices 3 (2001) 191.

[8] H. Suzuki and C.-M. Ho, IEEE Proc. Int. Conf. MEMS, 40 (2002).

[9] M. Tondra et al. IEEE Trans. Magnetics 37 (2001) 2621.

[10] T. Deng et al. Appl. Phys. Lett. 78 (2001) 1775.

[11] A. Rida, V. Fernandez, and M. A. M. Gijs, Appl. Phys. Lett. 83 (2003) 2396.

[12] M. Zborowski et al. J. Magn. Magn. Mater. 194 (1999) 224. 
[13] R. Gerber and R. R. Birss, High Gradient Magnetic Separation, Research Studies Press, Chichester, 1983.

[14] D. R. Lide (Ed.), CRC Handbook of Chemistry and Physics, $75^{\text {th }}$ Edition, CRC Press, Boca Raton, 1994 


\title{
CHAPTER 3. GIANT MAGNETORESISTANCE MONITORING OF MAGNETIC PICODROPLETS IN AN INTEGRATED MICROFLUIDIC SYSTEM
}

\author{
A paper published in Applied Physics Letters ${ }^{\dagger}$ \\ Nikola Pekas and Marc D. Porter \\ Institute for Combinatorial Discovery, Departments of Chemistry and Chemical \\ Engineering, and Ames Laboratory-USDOE, Iowa State University, Ames, Iowa 50011 \\ Mark Tondra, Anthony Popple, and Albrecht Jander \\ NVE Corporation, Eden Prairie, Minnesota 55433
}

\begin{abstract}
This Letter describes the integration of Giant MagnetoResistance (GMR) sensors with a microfluidic system for the velocity and size monitoring, and enumeration of flowing magnetic entities. We have fabricated a microdevice that enables: (1) controlled formation of picoliter-sized droplets of a ferrofluid separated by a non-magnetic oil; and (2) continuousflow sensing of these ferrofluid droplets. We show that the flow velocity, droplet size, and droplet-formation frequency can readily be determined from the GMR response. These results are validated by comparisons to fluorescence microscopy data.
\end{abstract}

\section{Introduction}

Giant magnetoresistors (GMRs) undergo a large change in electrical resistance as a function of magnetic field strength. ${ }^{1}$ GMRs are microfabricated structures commonly utilized to read computer hard disks, and have been employed for detection of magnetic particle labels in various surface-based bioassays. ${ }^{2-4}$ This Letter details the integration of GMRs with a microfluidic system for the size monitoring and enumeration of flowing magnetic droplets, extending a recent report on the flow velocity detection of magnetic particles. ${ }^{5}$ To these ends, we have: (1) fabricated a microdevice that enables formation of picoliter droplets of a waterbased ferrofluid separated by a nonmagnetic oil; (2) monitored the GMR response that results

\footnotetext{
${ }^{\dagger}$ Reprinted with permission from Pekas, N., Porter, M. D., Tondra, M., Popple, A. \& Jander, A. Giant magnetoresistance monitoring of magnetic picodroplets in an integrated microfluidic system. Applied Physics Letters 85, 4783-4785 (2004). Copyright 2005, American Institute of Physics.
} 
from this magnetically-discrete flow pattern; and (3) compared these data to fluorescence microscopy images to assess device performance.

Several lab-on-a-chip devices employ immiscible fluids in which one phase is, or can be rendered, magnetic. For example, micropumps have been devised ${ }^{6,7}$ that use ferrofluid plugs as pistons. An immiscible ferrofluidic phase may also be valuable in systems employed for controlled emulsification, ${ }^{8,9}$ combinatorial synthesis, ${ }^{10}$ or biochemical screening ${ }^{10}$ within discrete picoliter droplets. In each case, incorporation of a micromagnetic sensing strategy would potentially eliminate the need for off-chip monitoring instrumentation as part of a feedback mechanism for flow control. ${ }^{5}$

\section{Fabrication and experimental setup}

Figure 1 shows our integrated silicon-based platform. It includes three sets of sensors, each composed of four $20 \times 4-\mu \mathrm{m}$ spin-valve GMRs ${ }^{11}$ wired as Wheatstone bridges. Each spin valve is a $\mathrm{Ta}(40 \AA)-\mathrm{NiFeCo}(50 \AA)-\mathrm{Ta}(50 \AA)-\mathrm{NiFeCo}(40 \AA)-\mathrm{CoFe}(10 \AA)-\mathrm{Cu}(25 \AA)-$ $\mathrm{CoFe}(40 \AA)-\mathrm{CrPtMn}(325 \AA)$ stack, where the atomic percentages of the alloy compositions are $\mathrm{NiFeCo} 64 / 16 / 20$, CoFe 95/5, and CrPtMn 45/10/45. In each bridge, two GMRs act as sense-resistors and are centered directly under a microfluidic channel (13- $\mu \mathrm{m}$ wide; $18-\mu \mathrm{m}$ deep) that was lithographically defined by the full removal of an $18-\mu \mathrm{m}$ thick Cyclotene polymer (Dow Chemical). The other two GMRs serve as reference resistors, which are located beneath the Cyclotene layer [Fig. 1(a)] and separated from the edge of the neighboring sense GMRs by $26 \mu \mathrm{m}$.

Two more steps complete the microfluidic system construction. First, the device is sputter-coated with 300-nm passivation layer of silicon nitride, which is then oxygen-plasma activated ( $60 \mathrm{~s}$ at $0.02 \mathrm{~W} / \mathrm{cm}^{2}$ and 1 torr), along with the bottom of a poly(dimethylsiloxane) (PDMS) lid. ${ }^{12}$ The lid, built by micromolding, ${ }^{12}$ contains a channel that is $30-\mu \mathrm{m}$ wide and $25-\mu \mathrm{m}$ deep. The channels are then aligned perpendicularly to each other and the two pieces are brought into light conformal contact, which spontaneously forms a leak-tight seal. The resulting fluidic system therefore contains a vertical junction located at the intersection of the two channels, as illustrated in Fig. 1(c). This approach facilitates the development of other microfluidic formats by simply changing the structure in PDMS. We note that the siliconnitride coating separates the top of each sense GMR from the bottom of the flow channel by $300 \mathrm{~nm}$. 

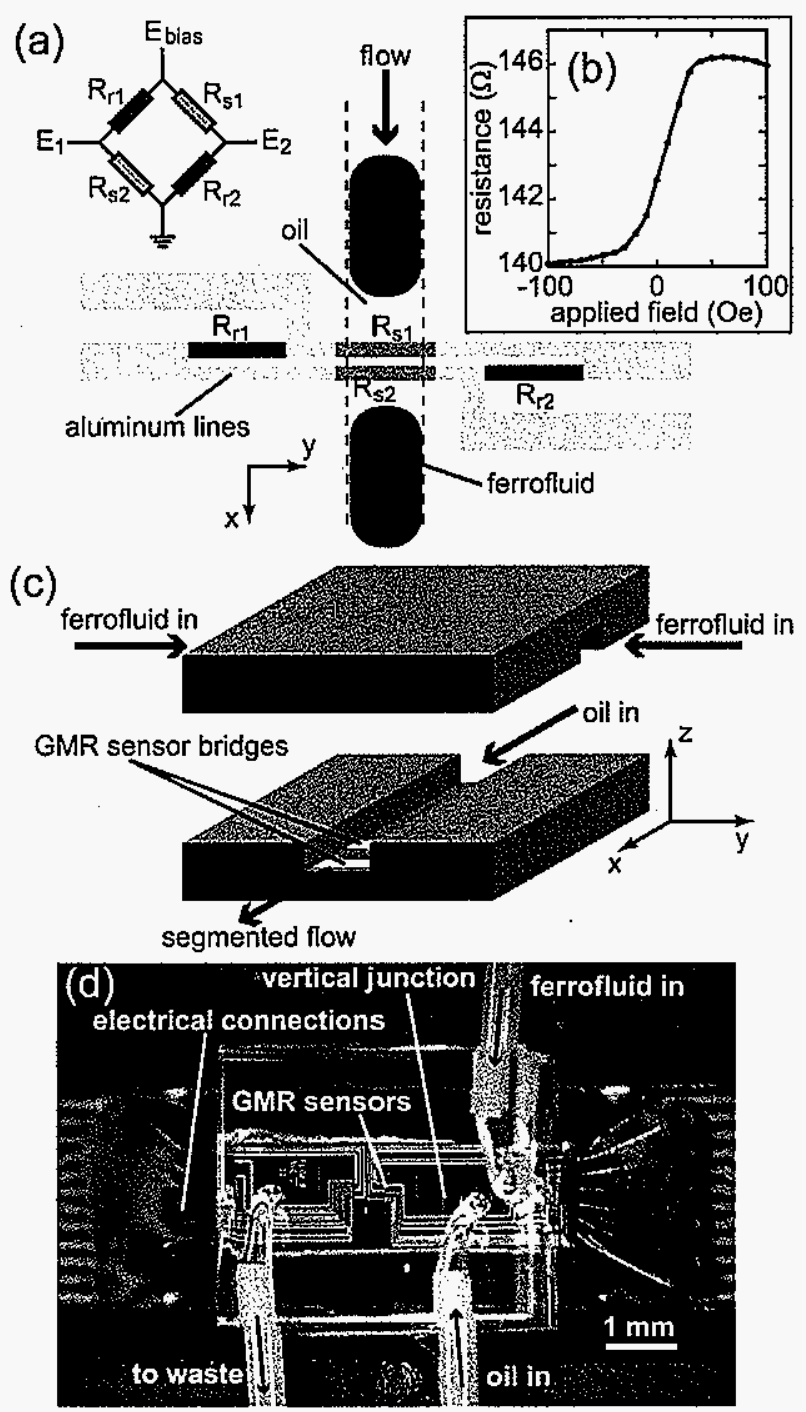

FIG. 1. (a) Top-view schematic of the GMR flow-sensor design; (b) the Wheatstone bridge transfer curve (twopoint measurement of the voltage drop between $E_{1}$ and $E_{2}$ upon sourcing a constant current at each point); (c) exploded view of the junction in which alternating droplets of oil and water-based ferrofluid are formed; and (d) a photograph of the finished device. The ferrofluid flow splits into two channels that recombine at the top of the vertical junction in Fig. 1(c). In each sensor, two sense $\left(R_{51}\right.$ and $\left.R_{52}\right)$ and two reference $\left(R_{r 1}\right.$ and $\left.R_{r 2}\right)$ GMRs form a Wheatstone bridge. The bridge bias voltage $\left(E_{\text {bias }}\right)$ is $+0.5 \mathrm{~V}$, with $15 \mathrm{Oe}$ applied along the GMR sense direction (x-axis).

After sealing, the device is wire bonded to a printed-circuit board. It is then connected to syringe pumps by microcapillaries mounted via access ports that were formed in the lid by affixing preformed polymeric inserts in the mold prior to PDMS casting. Two syringe pumps were used to meter the ferrofluid and oil. The ferrofluid (aqueous suspension of 10-nm magnetite particles, Ferrotec EMG 507) is diluted to $1.2 \%$ v/v magnetite with a Tris-buffered fluorescein solution ( $3 \mathrm{mM}$ after mixing, $\mathrm{pH} \mathrm{8.0)} \mathrm{for} \mathrm{fluorescence} \mathrm{imaging.} \mathrm{The} \mathrm{oil} \mathrm{phase}$ was prepared by adding $10 \%(\mathrm{v} / \mathrm{v})$ tridecafluorooctanol to perfluorodecalin. ${ }^{13}$ The two 
immiscible liquids, when encountering each other at the channel crossing, form a flow pattern of alternating droplets. ${ }^{8}$ The relative sizes of these droplets are dictated by the difference in the flow rates of the two liquids; the droplet-formation frequency is determined by the total flow rate. Finally, the device is mounted between the poles of a miniature electromagnet in order to apply an external field along the "sense" GMR direction $\left(H_{x}\right.$ ), which is perpendicular to the long axis of the GMR strips. Sweeping-field measurements [Fig. 1(b)] showed that the spin-valves have a highly linear magnetoresistive response, with a sensitivity of $0.077 \% / \mathrm{Oe} .{ }^{14}$ All subsequent experiments were carried out at 15 Oe.

\section{Results and discussion}

Figure 2 presents a microscopic image of the GMR network and a set of responses for two neighboring bridges. The image shows that the ferrofluid plugs are $66 \mu \mathrm{m}$ long in the topmost channel (17- $\mu \mathrm{m}$ wide) and elongate to $86 \mu \mathrm{m}$ upon turning into the narrower $(13-\mu \mathrm{m}$ wide) channel aligned across the bridges.
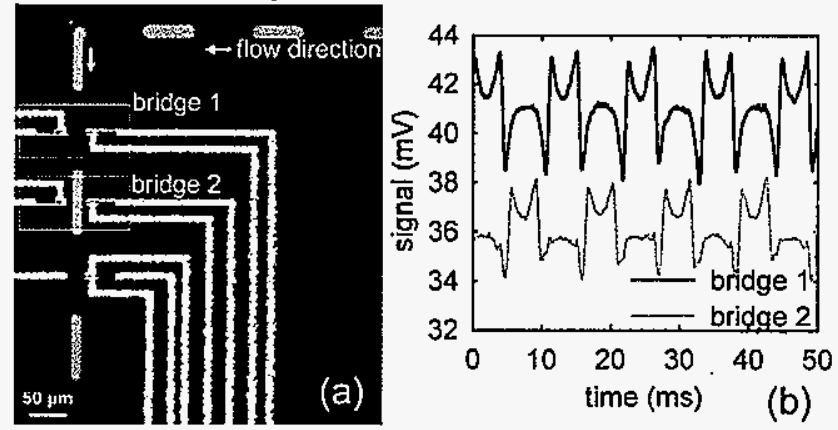

FIG. 2. (a) Fluorescence micrograph of alternating ferrofluid (bright segments) and oil droplets flowing across the sense-GMRs; and (b) the corresponding responses of bridge 1 and bridge 2 . The two bridges are separated by $100 \mu \mathrm{m}$. The DC-determined voltage drop $\left(E_{1}-E_{2}\right)$ across each bridge is amplified (gain $=29$ ) to span the input range of an analog-to-digital converter, digitized at $100 \mathrm{kHz}$, and averaged with a 20-point moving interval. The fluorescence micrograph is combined with the dark-field micrograph to visualize the flow channel and aluminum interconnects. The GMRs are not visible. The ferrofluid-to-oil flow-rate ratio was close to unity, with a total rate of $280 \pm 20 \mathrm{~nL} / \mathrm{min}$. The offsets in experimental data reflect the imperfectly balanced resistances of aluminum leads within the bridge, along with small differences in the GMR response curves.

Based on the plug length and channel dimensions, each plug has a volume of $20 \pm 3 \mathrm{pL}$. A plug therefore contains $(5 \pm 1) \times 10^{8} 10$-nm particles. The bridge outputs, however, have a complex shape, and we performed a finite-element analysis of the fringe field for a ferrofluid plug to gain insight into the origin of the pattern. The model was developed using AMPERES software, based on the plug dimensions of $86 \times 13 \times 18 \mu \mathrm{m}$, and an external field of 15 Oe applied along the $x$-axis. Based on vendor specifications, the magnetic susceptibility of diluted ferrofluid was estimated to be 0.25 . 
The calculated values for $H_{x}$ at the GMR surface (i.e., $300 \mathrm{~nm}$ below the plug) are shown in Fig. 3(a). The expected bridge response to the movement of this field across the senseGMRs is given in Fig. 3(b) and represents the simulated voltage-drop across a bridge due to passage of a plug that is based on the calculated magnetic field, bridge geometry, the GMR sensitivity, and the experimental DC amplification.
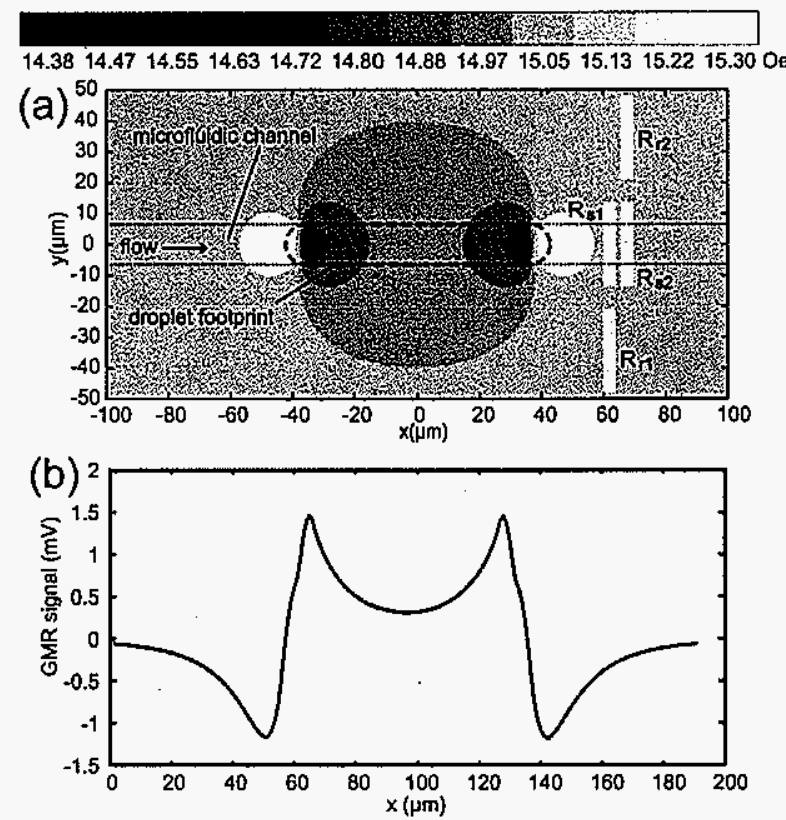

FIG. 3. (a) Theoretical assessment of the total field component along the sense direction, $H_{x}$, i.e., the sum of the external 15 Oe-field applied along the $x$-axis and the field induced in the ferrofluid plug. The plot shows data for a plane located $300 \mathrm{~nm}$ below the droplet (sensor plane), and ferrofluids with a susceptibility of 0.25 . The plot also shows the footprint of a ferrofluid droplet and the positions of the sense and reference GMRs. (b) Calculated voltage drop across the Wheatstone bridge due to droplet passage. The calculation is based on the GMR sensitivity of $0.077 \% / \mathrm{Oe}$, bridge bias voltage of $+0.50 \mathrm{~V}$, and amplifier gain of 29 .

Prior to contact with the plug field, the response represents only the effect of the constant external field. As the plug approaches the sense-GMRs, the field increases, which, in turn, gives rise to an increase in the sense-GMR resistances and therefore a decrease in the voltage drop $\left(E_{1}-E_{2}\right)$ across the bridge. As the front of the plug moves past the sense GMRs, the field rapidly decreases and the response undergoes a corresponding increase. The response then slowly decreases as the middle of the plug flows across the sense GMRs. Movement of the back half of the plug across the sense GMRs reverses the observed response pattern.

The flow velocity can be determined by cross-correlating the response from the two bridges. Since, the distance between bridges is $100 \mu \mathrm{m}$, the cross-correlation peak position represents the time lag between the two signals. For the data in Fig. 2(b), the calculated 
velocity equals $19.0 \pm 0.4 \mathrm{~mm} / \mathrm{s}$, which is in reasonable agreement with the expected flow velocity of $21 \pm 2 \mathrm{~mm} / \mathrm{s}$ based on the pump settings.

The droplet length can also be deduced from these data by defining the magnetic footprint of a droplet as the interval flanked by the maximum changes in the GMR signal. To find an average magnetic footprint, we first calculated the squared derivative of the signal and then applied an autocorrelation algorithm to determine the temporal shift between neighboring maxima in the squared-derivative data. Since the flow velocity is known, the time interval can be readily converted into a length scale. Thus, the magnetic footprint equaled $84 \pm 3 \mu \mathrm{m}$, whereas the optically determined plug length was $86 \pm 3 \mu \mathrm{m}$. Another example is given in Fig. 4, where flow-rate ratio of ferrofluid to oil was ca. five. In this case, the fluorescence image yields a ferrofluid length of $186 \pm 3 \mu \mathrm{m}$. The magnetic footprint was $184 \pm 3 \mu \mathrm{m}$. Finally, the droplet frequency can be determined by a fast-Fourier transformation of the acquired signal, and values obtained in this way matched those derived from the flow rates and droplet sizes.
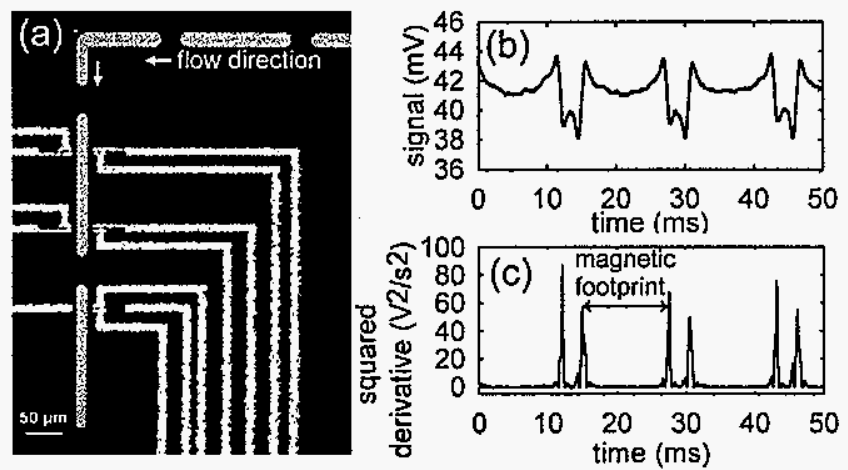

FIG. 4. (a) Fluorescence micrograph; (b) sensor response; and (c) magnetic-footprint determined from the response. The size of ferrofluid droplets reflects a ferrofluid-to-oil flow-rate ratio of $c a$. five, which elongates the ferrofluid droplets with respect to the oil, and is apparent in the GMR signal.

Although our device was designed to use an "off-chip" field for ferrofluid magnetization, droplet passage was detectable without this field (data not shown). The observed response, which was $20 \%$ of that in Fig. 2 , is attributed to the current sourced (4 mA) through a bridge, which would yield a field of $\sim 2$ Oe within a few micrometers of the GMRs. This finding, while preliminary, suggests that it may be possible to use microfabricated current lines in the device design to induce the requisite magnetic fields, thereby eliminating the need for externally mounted electromagnets.

In summary, we have demonstrated the successful integration of GMR sensors with a microfluidic system, and reliable sensing of flowing ferrofluid droplets generated in situ 
upstream of the sensors. Flow properties inferred from the sensor signal are in reasonable agreement with the results obtained by fluorescence microscopy. We are now exploring the scope of this concept for the detection and enumeration of magnetically tagged biological analytes. Support from NSF's XYZ-on-a-Chip initiative, the W. M. Keck Foundation, and DARPA's BioMagnetICs program is acknowledged. The Ames Laboratory is operated for the U.S. Department of Energy by Iowa State University.

\section{References cited}

${ }^{1}$ K. E. Johnson, J. Appl. Phys. 87, 5365 (2000).

${ }^{2}$ D. L. Graham, H. A. Ferreira, P. P. Freitas, and J. M. S. Cabral, Biosens. Bioelectron. 18, 483 (2003).

${ }^{3}$ M. Tondra, M. D. Porter, and R. J. Lipert, J. Vac. Sci. Technol. A 18, 1125 (2000).

${ }^{4}$ J. C. Rife, M. M. Miller, P. E. Sheehan, C. R. Tamanaha, M. Tondra, and L. J. Whitman, Sens. Actuators A 107, 209 (2003).

${ }^{5}$ H. A. Ferreira, D. L. Graham, P. Parracho, V. Soares, and P. P. Freitas, IEEE Trans. Magn. 40, 2652 (2004).

${ }^{6}$ A. Hatch, A. E. Kamholz, G. Holman, P. Yager, and K. F. Böhringer, J. Microelectromech. Syst. 10, 215 (2001).

${ }^{7}$ Y. Melikhov, S. J. Lee, D. C. Jiles, D. H. Schmidt, M. D. Porter, and R. Shinar, J. Appl. Phys. 93, 8438 (2003).

${ }^{8}$ T. Thorsen, R. W. Roberts, F. H. Arnold, and S. R. Quake, Phys. Rev. Lett. 86, 4163 (2001).

${ }^{9}$ D. R. Link, S. L. Anna, D. A. Weitz, and H. A. Stone, Phys. Rev. Lett. 92, 054503-1 (2004).

${ }^{10}$ B. Zheng, L. S. Roach, and R. F. Ismagilov, J. Am. Chem. Soc. 125, 11170 (2003).

${ }^{11}$ Z. Qian, J. M. Daughton, D. Wang, and M. Tondra, IEEE Trans. Magn. 39, 3322 (2003).

${ }^{12}$ D. C. Duffy, J. C. McDonald, O. J. A. Schueller, and G. M. Whitesides, Anal. Chem. 70, 4974 (1998).

${ }^{13}$ H. Song, J. D. Tice, and R. F. Ismagilov, Angew. Chem. Int. Ed. 42, 767 (2003).

${ }^{14}$ Z. Qian, D. Wang, J. Daughton, M. Tondra, E. Lange, C. Nordman, A. Popple, J. Myers, and J. Schuetz, J. Appl. Phys. 93, 6870 (2003). 


\title{
CHAPTER 4. QUANTITATIVE MAGNETIC SENSING IN MICROCHANNELS: FIGURES OF MERIT AND IMPLICATIONS FOR MAGNETIC FLOW CYTOMETRY
}

\author{
A paper to be submitted to Lab on a Chip \\ Nikola Pekas and Marc D. Porter \\ Institute for Combinatorial Discovery, Departments of Chemistry and Chemical \\ Engineering, and Ames Laboratory-USDOE, Iowa State University, Ames, Iowa 50011 \\ Mark Tondra, Anthony Popple, and Albrecht Jander \\ NVE Corporation, Eden Prairie, Minnesota 55433
}

\begin{abstract}
We describe quantitative and rapid measurements of picoliter-sized droplets of magnetite nanoparticle suspensions in a microfluidic system with embedded spin-valve Giant Magnetoresistance (GMR) sensors. The limits of detection were determined and compared to numerical models, suggesting that the present system is capable of detecting individual ferromagnetic beads in flow. The experimental results confirmed this conclusion. The comparison also defines some of the key design requirements for the creation of a magnetic flow cytometer for detection of superparamagnetic labels or magnetotactic bacteria.
\end{abstract}

\section{Introduction}

Flow cytometry is a well established technique for high-speed, multiparametric analysis of single cells. ${ }^{1,2}$ In a conventional flow cytometry, the cells are selectively labeled with fluorescently tagged antibodies, suspended in the carrier buffer, and hydrodynamically focused by a coaxial liquid sheath to pass precisely through the detection volume in singlefile flow. The detection scheme is realized by laser excitation and light-scatter and fluorescence signal collection. Recent miniaturization trends in analytical sciences have not bypassed flow cytometry. ${ }^{3-6}$ Some of the potential advantages of microfluidic flow cytometry $(\mu F C M)$ include: portability; multiplexing; and integration with other lab-on-a-chip functionalities (e.g., sample preparation and concentration). Most of the work in the $\mu \mathrm{FCM}$ area relies on optical or impedance detection. Optical detection methods, while versatile, require a complex network of light sources, filters, and detectors, making microfabricated integration into a lab-on-a-chip format difficult. In fact, most of the work with $\mu \mathrm{FCM}$ 
described to date utilize external detection setups ${ }^{5-7}$ Impedance-based methods are based on microfabricated electrodes, and therefore promise a higher potential for integration with microfluidic systems. ${ }^{3,4}$ However, all described impedance-based schemes are presently label-free, and therefore offer little specificity.

One exciting detection prospect lies with microfabricated Giant Magnetoresistance (GMR) sensors, which respond to magnetic field with a change in electrical resistance. ${ }^{8-10}$ Their inherently small size, high sensitivity, compatibility with present lab-on-a-chip fabrication procedures, and fast response make them exceptionally attractive as detection elements in $\mu \mathrm{FCM}$. Moreover, GMRs can be configured in a Wheatstone bridge electrical circuit network, with the response to the changes in magnetic fields followed simply by a change in the resistance of the network. Detection and molecular specificity in such a platform could therefore be achieved by utilizing magnetic micro- or nanoparticles after they have been modified with recognition elements (antibodies or aptamers). These types of particles are widely available, and have previously been used in cell separation and enrichment procedures. ${ }^{11-13}$

As a step towards realizing this possibility, this paper describes quantitative measurements and determinations of the limits of detection for a microfluidic system with embedded GMR sensors, and discusses the implications for the design of a magnetic flow cytometer. We first determined the limit of detection by monitoring the sensor response to series of flowing, picoliter-sized droplets of different concentrations of 10-nm magnetite particles. Next, we calculated the magnetic field profile resulting from this lowest detectable. concentration, thus expressing the detection limit in terms of the average detectable change in the field across the sensing area of the GMR. Finally, we compared the "field limit of detection" to the numerical models describing the magnetic field profiles for various targets: a superparamagnetic bead, a ferromagnetic bead, and a magnetosome ${ }^{14}$ of a magnetotactic bacterium. The findings not only substantiate and extend our earlier theoretical work on magnetic-readout, surface-bound immunoassays, ${ }^{15}$ but also point to the possibility for the detection of individual magnetic targets in a microfluidic flow stream. Indeed, the present design is shown to be effective to sense the presence of individual, 4- $\mu \mathrm{m}$ ferromagnetic beads in flow. This study also defines design requirements and future steps towards the creation of a magnetics-based flow cytometer.

\section{Experimental setup and numerical models}

The experimental setup has been described earlier. ${ }^{16}$ Briefly, the microfluidic chip features three sensor sets, each of which is composed of four $20 \times 4-\mu \mathrm{m}$ spin valve GMRs 
wired in a Wheatstone bridge configuration (see Figure 1). In each bridge, two GMRs act as sense-resistors $\left(R_{\mathrm{s} 1}\right.$ and $\left.R_{\mathrm{s} 2}\right)$, and are positioned directly beneath a microfluidic channel that was lithographically defined in an $18-\mu \mathrm{m}$ thick benzocyclobutene (BCB) polymer. The channel width was $13 \mu \mathrm{m}$. The other two GMRs in each bridge serve as reference resistors $\left(\mathrm{R}_{\mathrm{r} 1}\right.$ and $\left.\mathrm{R}_{\mathrm{r} 2}\right)$, and are located beneath the $\mathrm{BCB}$ layer. The separation distances between the first, second, and the third bridge are $\Delta x_{1-2}=106 \mu \mathrm{m}, \Delta x_{2-3}=100 \mu \mathrm{m}$, and $\Delta x_{1-3}=206 \mu \mathrm{m}$. The sensors were passivated by sputtering a 300-nm layer of silicon nitride, which defines the bottom of the flow channel. The channel was sealed by bonding a poly(dimethylsiloxane) (PDMS) lid to the top surface. The lid also included a flow channel that intersected at $90^{\circ}$ with respect to the channel in the underlying BCB layer to form a vertically-offset fluidics junction that is situated $\sim 800 \mu \mathrm{m}$ upstream of the first sensor bridge. The cross-section of the PDMS channel was $30 \times 30 \mu \mathrm{m}$. When a water-based ferrofluid and an oil phase are pumped through these two channels, a segmented flow of alternating ferrofluid and oil droplets is formed at the junction. ${ }^{17,18} \mathrm{~A}$ voltage drop across each of the bridges $\left(\mathrm{E}_{1}-\mathrm{E}_{2}\right)$ was amplified to span the input range of a 16-bit analog-to-digital converter. The available amplification factor was different for each bridge, as determined by the dc offset due to the imperfectly balanced resistances of the electrical interconnects within a bridge. ${ }^{9}$ Data processing was performed using a National Instruments DIAdem software package.

Different concentrations of magnetite nanoparticles were obtained by diluting an aqueous ferrofluid EMG 507 (FerroTec) with water. This ferrofluid consists of 10-nm magnetite particles that are stabilized by a surfactant, and can be diluted with either water or ethanol. The original concentration of magnetite was $1.8 \%$ by volume, or $3.4 \times 10^{16}$ particles $/ \mathrm{mL}$. The oil phase was prepared by adding tridecafluorooctanol (TDFO) to perfluorodecalin to the final TDFO concentration of $10 \%$ by volume. ${ }^{19}$

Fluorescent ferromagnetic beads (Nile Red fluorophore, $20 \% \mathrm{CrO}_{2} \mathrm{v} / \mathrm{v}$ ) were obtained from Spherotech, and had an average diameter of $4.4 \mu \mathrm{m}$. Prior to experiments, the beads were diluted in water with $0.05 \%$ Tween- 80 surfactant, to the final concentration of $4 \times 10^{3}$ beads $/ \mu \mathrm{L}$, and magnetized by applying a field of $80 \mathrm{kA} \cdot \mathrm{m}^{-1}$. Based on the product specifications, this procedure yields a remnant induction of approximately $10 \mathrm{mT}$. In all cases, syringe pumps with 50- $\mu \mathrm{L}$ Gastight syringes (Hamilton) were used to deliver solutions to devices via polymeric capillary tubing (Upchurch Scientific). For bead-detection experiments, microchannels in PDMS were not needed, and the chip featured one fluidic inlet and one outlet, with the $\mathrm{BCB}$ channel structure identical to that described above. 

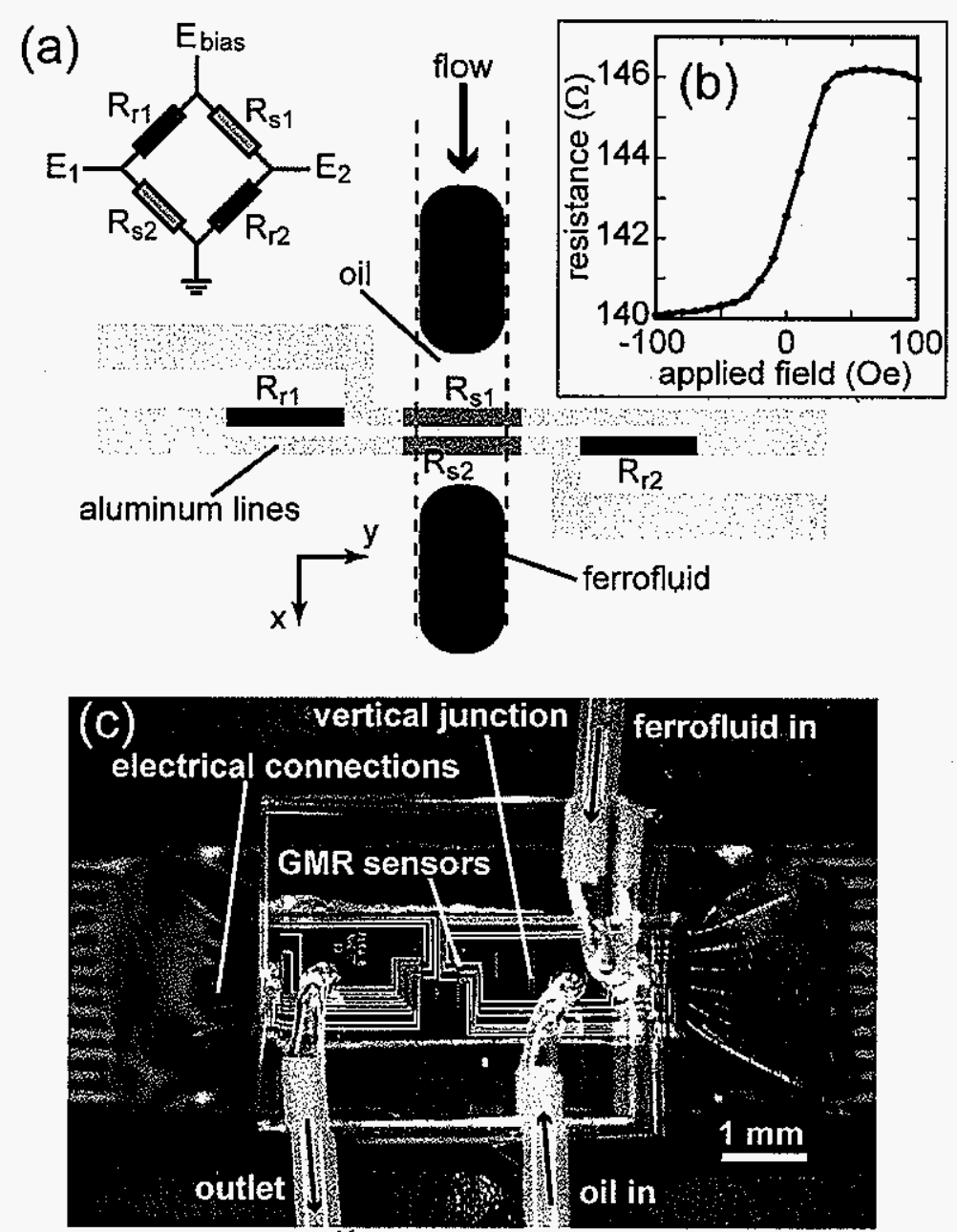

Figure 1. (Adapted from our previous work.) ${ }^{16}$ (a) Top-view schematic of the GMR flow detector. Four sensors are wired in a Wheatstone bridge configuration, with two sensors serving as sense resistors $\left(R_{s 1}\right.$ and $\left.R_{s 2}\right)$, and the other two sensors functioning as reference resistors $\left(R_{r 1}\right.$ and $\left.R_{r 2}\right)$. The GMRs are sensitive to the $x$ component of the magnetic field. The ferrofluid droplets are formed upstream from sensors by merging together an aqueous ferrofluid and an oil phase flow (not shown). ${ }^{17,18}$ (b) The transfer curve obtained from a two-point measurement of the resistance between $E_{1}$ and $E_{2}$. Note that the useful range of the sensor response is approximately $\pm 20 \mathrm{Oe}\left( \pm 1.6 \mathrm{~A} \cdot \mathrm{m}^{-1}\right)$. The sensitivity is $0.08 \% / \mathrm{Oe}$. (c) A photograph of the assembled device. The ferrofluid flow splits into two streams that recombine at the top of the vertical junction. The segmented ferrofluid/oil two-phase flow is formed and guided over the GMR sensors.

Three-dimensional numerical models were developed in three dimensions using FEMLAB multiphysics package (Comsol). Ferrofluid droplets and superparamagnetic particles were represented as magnetizable objects placed in a non-magnetic medium, with an external field applied along the $x$-axis direction (see Figure 1). Ferromagnetic beads and magnetosomes were represented as magnetic objects with a remnant induction, immersed in a non-magnetic medium. Since coercivities of magnetosomes ${ }^{20}$ and ferromagnetic beads (vendor specified) are much higher than the fields used to bias sensors and to align particle 
moments along the sense direction without saturating the sensor, external fields are not expected to noticeably affect the magnetic profiles of the ferromagnetic beads or magnetosomes, and are therefore neglected.

\section{Results and discussion Quantitative measurements}

An example of the response of two sensor bridges to the ferrofluid-oil segmented flow is shown in Figure 2, along with the cross-correlation function. The bridge bias voltage $E_{\text {bias }}$ was $+0.50 \mathrm{~V}$, with an applied excitation field $H_{\text {ext }}$ of $954.7 \mathrm{~A} \cdot \mathrm{m}^{-1}(12 \mathrm{Oe})$. The amplification of the bridge output $\left(E_{1}-E_{2}\right)$ was set to 51 . After the amplification step, the root-mean-square baseline noise measured in a 10 -ms interval was $V_{r m s}=200 \mu \mathrm{V}$. For a given ferrofluid droplet, the signal was determined as the difference between the averaged maximum and averaged minimum of the magnetic signature. Based on the time lag between the two signals, as determined from the cross-correlation peak, the flow velocity can be estimated. Droplet sizes can then determined from the magnetic footprints and the flow velocity.

The experiments were repeated for four concentrations of the ferrofluid [see Figure 3(a)]. For each concentration, signals for ten droplets were averaged to obtain the calibration plot shown in Figure 3(b). The lengths of the droplets varied from $\sim 33 \mu \mathrm{m}$ to $\sim 150 \mu \mathrm{m}$, as determined by the relative flow rates. ${ }^{17}$ While the amplitude of the signal varied strongly with the magnetite concentration, no significant dependence of the response with droplet size was found. The latter observation is consistent with our previous experiments. ${ }^{16}$

The limit of detection at a signal-to-noise ratio of 3 was found to be $0.13 \%$ magnetite by volume, or $2.5 \times 10^{15}$ particles $/ \mathrm{mL}$. Based on the measurements of susceptibility $(\chi)$ for ferrofluids of different concentrations, as provided by the vendor (see Figure 4), this concentration corresponds to $\chi=0.022$. 

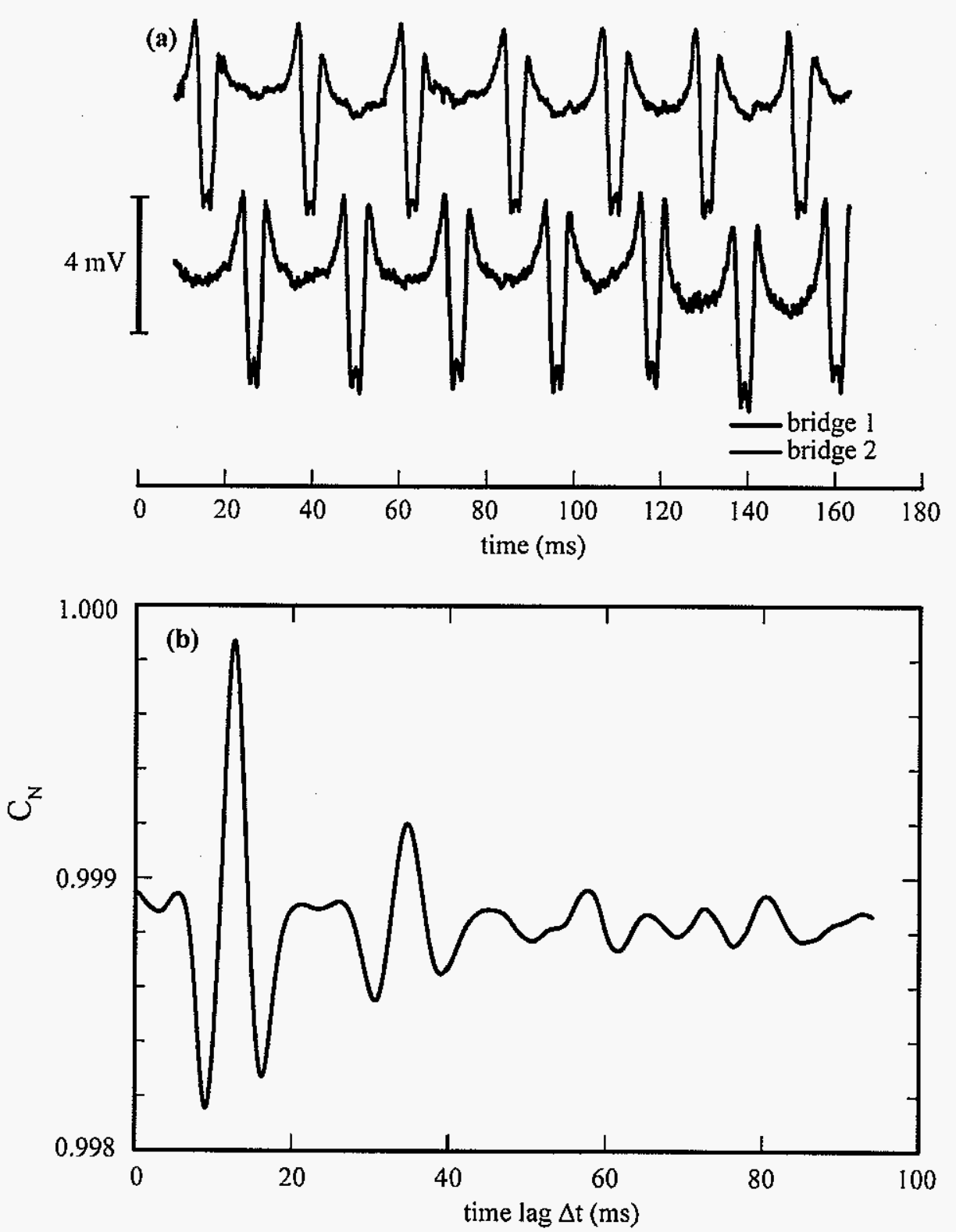

Figure 2. (a) Responses of two sensor bridges to the flow of magnetic droplets. The magnetite concentration was $0.9 \% \mathrm{v} / \mathrm{v}$, and the applied external field was $954.7 \mathrm{~A} \cdot \mathrm{m}^{-1}(12 \mathrm{Oe})$. The signal was amplified with the gain of 51 , and digitized at a $100 \mathrm{kHz}$ sampling rate. The two bridges are separated by $\Delta x_{1-2}=106 \mu \mathrm{m}$ in the flow channel. The plot shows 11-point moving average of the raw data. (b) Normalized cross-correlation function, $C_{N}$, of the raw data, showing the peak at $\Delta \mathrm{t}=12.6 \mathrm{~ms}$; this time lag corresponds to a droplet velocity of 7.9 $\mathrm{mm} / \mathrm{s}$. 

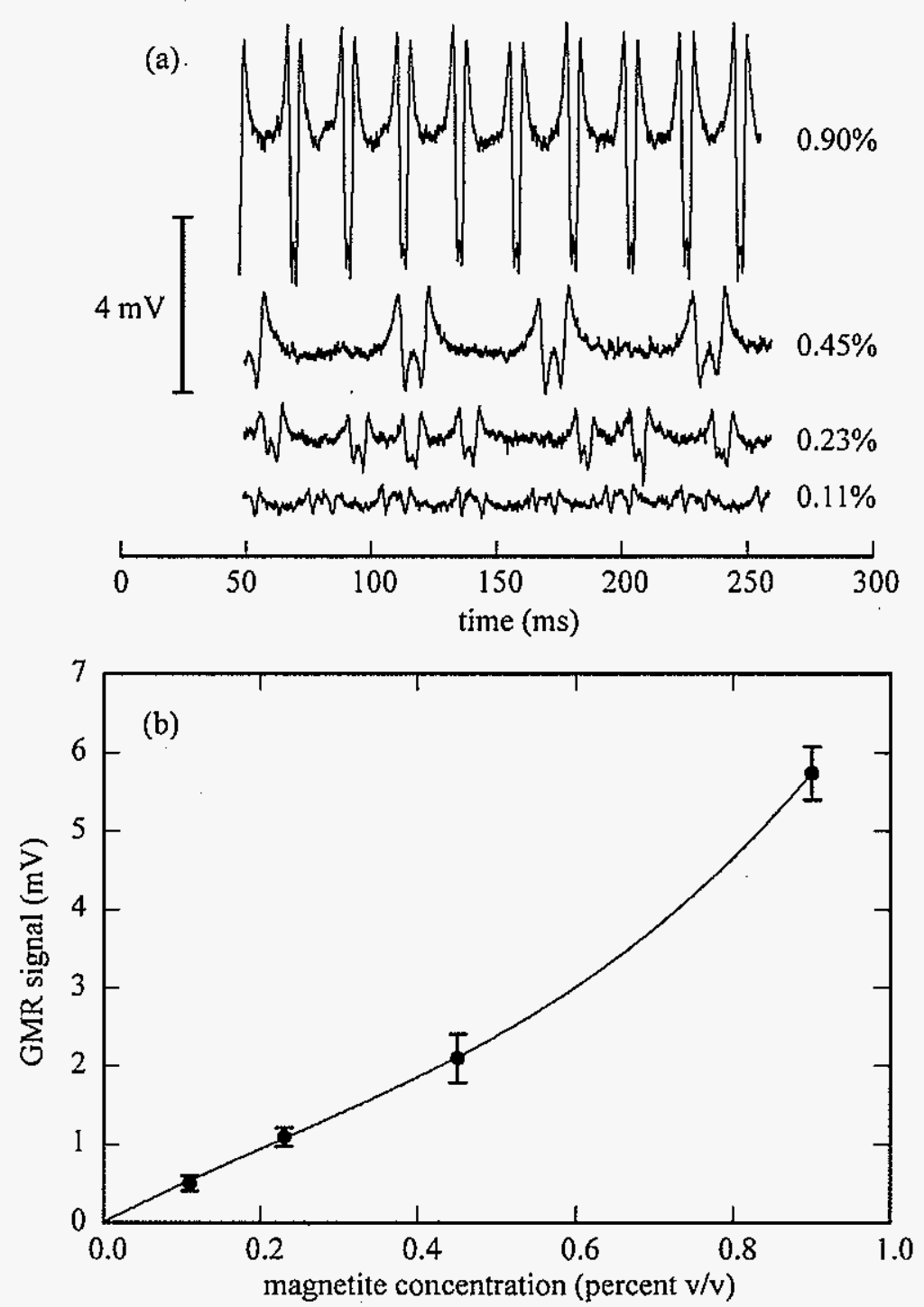

Figure 3. (a) Response of a GMR sensor bridge to series of droplets of four different concentrations of magnetite $(0.11 \%, 0.23 \%, 0.45 \%$, and $0.90 \% \mathrm{v} / \mathrm{v})$. The experimental parameters were identical to those described in Figure 4.2. (b) The calibration plot. Each signal value was determined as an average signal change for ten droplets. The error bars represent two standard deviations of the signals of ten droplets. The line is drawn as a guide to the eye, and represents the third-order polynomial fit: $y=5.2 x-3.5 x^{2}+5.4 x^{3}$; $\mathrm{R}^{2}=0.9999$. 


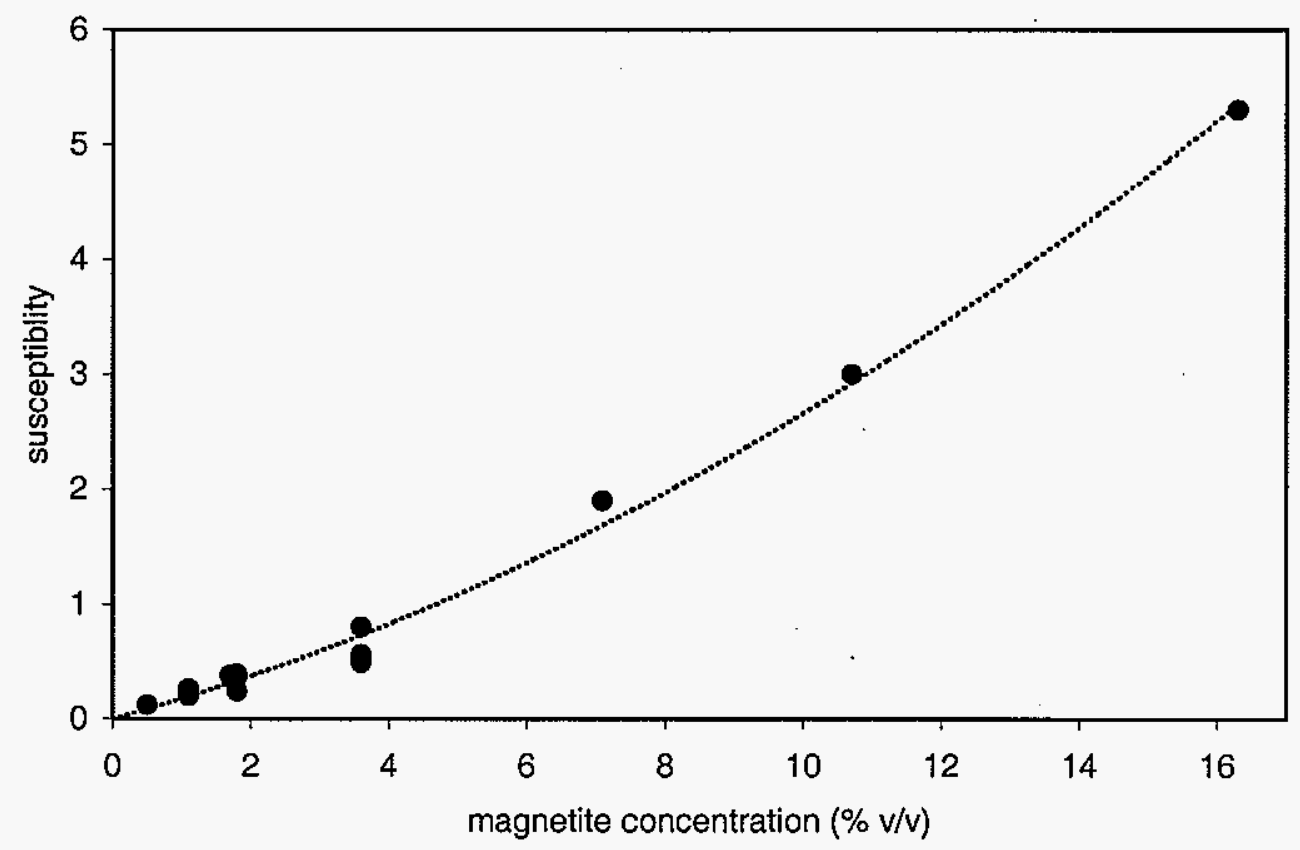

Figure 4. Compilation of the vendor-provided data on initial susceptibilities for ferrofluids of different concentrations of magnetite. The dashed line represents a second-order polynomial fit: $y=0.17 x+0.0098 x^{2} ; \mathrm{R}^{2}$ $=0.9928$.

In order to express the limit of detection in more universal terms of an average change of field across the active area of the sensor, we constructed a three-dimensional model of a magnetic droplet with the susceptibility of 0.022 , and determined the field profile in the sensor plane laying $300 \mathrm{~nm}$ beneath the droplet, since the actual devices featured a $300-\mathrm{nm}$ passivation layer of silicon nitride over sensors. The droplet cross-section was identical to that of a channel $(13 \times 18 \mu \mathrm{m})$, and the length was varied from 33 to $150 \mu \mathrm{m}$. In agreement with the experimental findings, the field profile did not change significantly for different lengths of droplets in the model. The results are shown in Figure 5. Since our spin-valve GMRs respond to the component of the field that is perpendicular to the sensor length, ${ }^{16}$ this transverse field component can be integrated within the sensor footprint $S$ to determine the average field observed by the sensor, $H_{x}^{a v}$, for a given relative position of the droplet to the sensor:

$$
H_{x}^{a v}=\frac{\iint_{S} H_{x} d x d y}{\iint_{S} d x d y}=1.7 \mathrm{~A} \cdot \mathrm{m}^{-1}
$$


The maximum averaged deviation $(\Delta H)$ from the externally applied field of $H_{\mathrm{ext}}=954.7$ $\mathrm{A} \cdot \mathrm{m}^{-1}(12.0 \mathrm{Oe})$, for a $4 \times 20 \mu \mathrm{m}$ sensor, was found to be $\Delta H=\left|H_{e x t}-H_{x}^{a v}\right|=1.7 \mathrm{~A} \cdot \mathrm{m}^{-1}(0.021$ Oe).

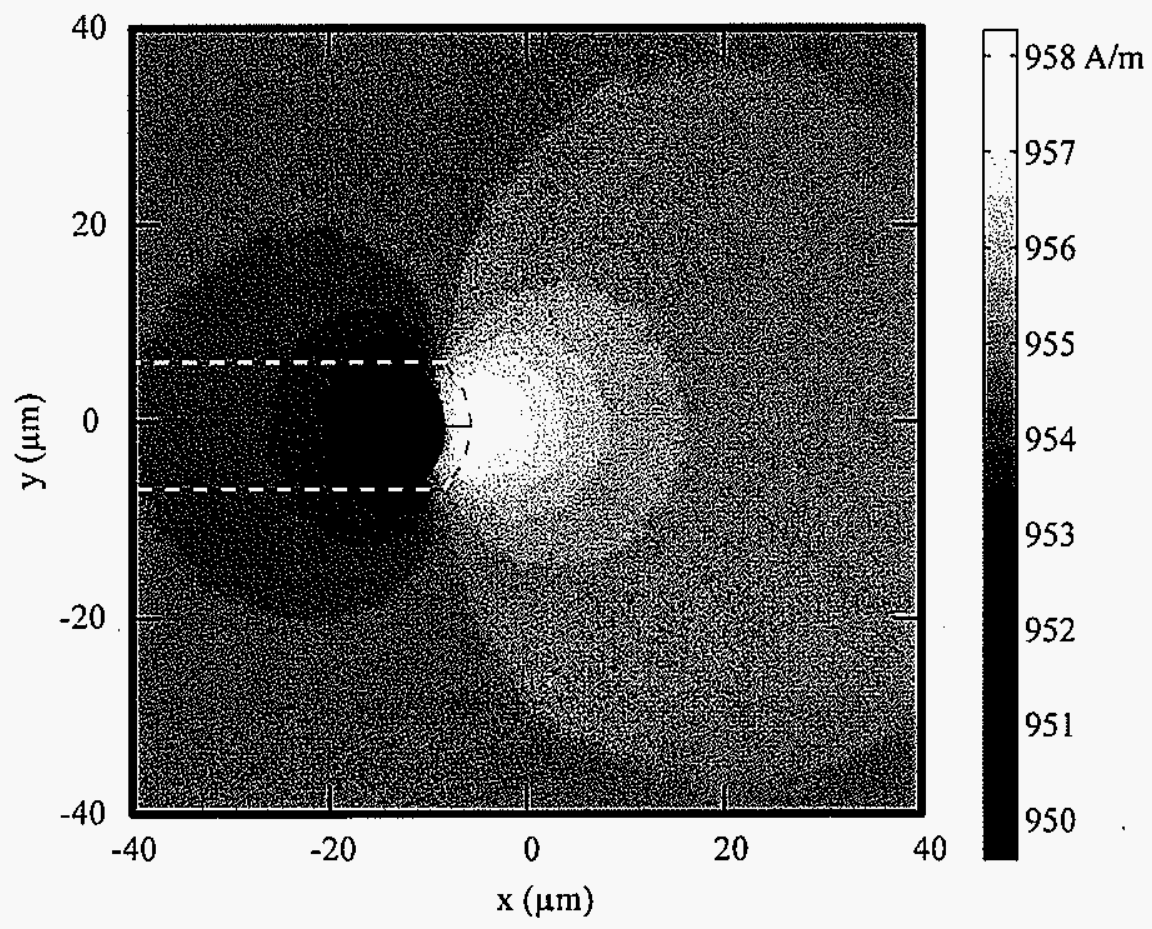

Figure 5. Top view close-up of the three-dimensional finite-element model of a ferrofluid droplet with a susceptibility of 0.022 . An external field of $954.7 \mathrm{~A} \cdot \mathrm{m}^{-1}$ is applied in the $x$-axis direction. The plot represents the $x$-component of the magnetic field in the sensor plane, $300 \mathrm{~nm}$ below the droplet. The dashed line depicts the droplet footprint.

\section{Finite-element modeling of magnetic targets of interest}

The following discussion compares expected field profiles for three different targets, averaged for three sizes of sensor strips, to the limit of detection determined above. The first target considered is a common superparamagnetic bead Dynal M270. An example of the finite-element modeling results is shown in Figure 6. Even at the bead-sensor separation gap of only $\Delta z=2 \mu \mathrm{m}$, the averaged field deviation from the externally applied excitation field amounts to around $\Delta H=0.64 \mathrm{~A} \cdot \mathrm{m}^{-1}$ - well beneath the present detection limit. One way to improve the observed signal would be to increase the externally applied field, and therefore the magnetization of the bead - however, existing spin valve sensors operate in a relatively low field $\left( \pm 1.6 \mathrm{kA} \cdot \mathrm{m}^{-1}\right)$. As a consequence, the ability to increase the magnetization of a bead by increasing the applied field is limited. The fabrication process could be modified to shift the sensor working point to higher fields, ${ }^{21}$ but technical challenges currently limit this option 
to a value of about $5 \mathrm{kA} \cdot \mathrm{m}^{-1}$. 22 Another way to obtain a stronger signal would be to fabricate sensors with smaller footprints, more suitable for targets in the micrometer size range. It is evident from Figure 4.6 that magnetic footprint of the bead is affecting only a portion of the available sensor area. For smaller objects, such as bacterial cells decorated with magnetic nanolabels, this problem only becomes more pronounced.

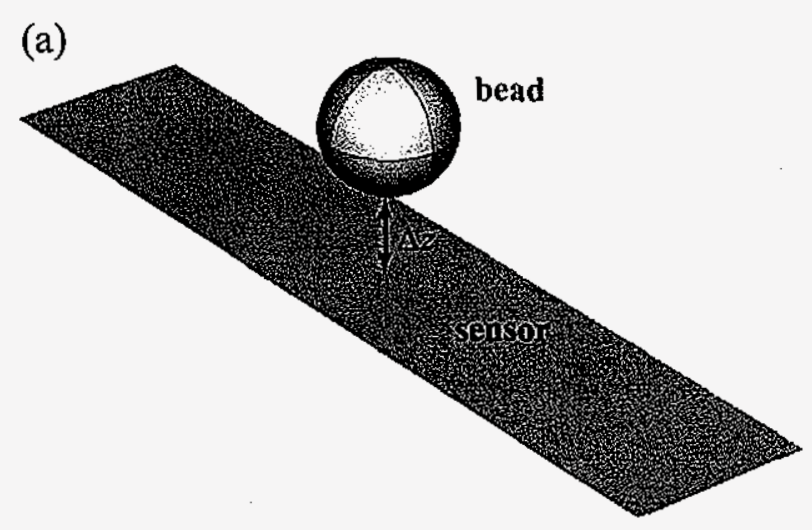

(b)

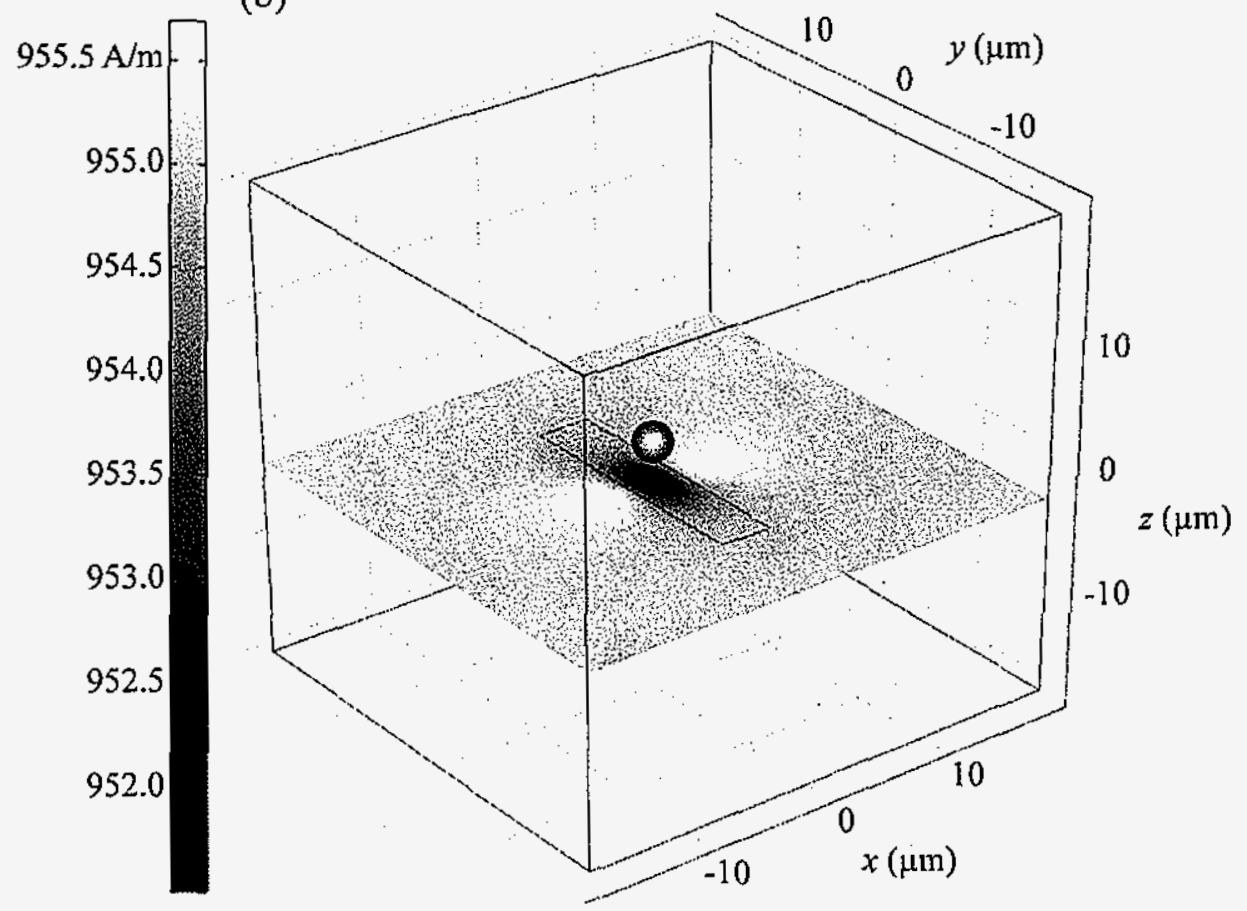

Figure 6. (a) A visual representation of a 2.8- $\mu \mathrm{m}$ Dynal M270 bead positioned at $\Delta z=2 \mu \mathrm{m}$ above the center of a $4 \times 20 \mu \mathrm{m}$ sensor strip. (b) Results of the finite-element modeling, for an external field of $954.7 \mathrm{~A} \cdot \mathrm{m}^{-1}$ applied in the $x$-axis direction. The plot represents the $x$-component of the total field, $H_{x}, 2 \mu \mathrm{m}$ beneath the bead surface. Also shown is a rectangular trace of a $4 \times 20 \mu \mathrm{m}$ sensor strip. The bead susceptibility was $\chi=0.17 .^{23}$ Based on this model, the averaged value of the $x$-component of the field is $H_{x}^{a v}=954.064 \mathrm{~A} \cdot \mathrm{m}^{-1}$. 
Magnetotactic bacteria represent another interesting target. ${ }^{14,20}$ The permanent magnetic moment of these cells originates from the intracellular presence of linear chains of biomineralized, single-domain particles of magnetite $\left(\mathrm{Fe}_{3} \mathrm{O}_{4}\right)$ or greigite $\left(\mathrm{Fe}_{3} \mathrm{~S}_{4}\right)$. The particles are 35-60 nm in diameter, and chains (or magnetosomes) usually contain 15-25 particles. The magnetization of the magnetosomes have been found to be comparable to the saturation magnetization values ( $B_{r}=0.6 \mathrm{~T}$ for magnetite), and magnetosome behaves as a single dipole, since stray fields from individual particles are negligible for long chains. ${ }^{20}$ The model predicts that a typical magnetosome $(40 \times 50 \times 1000 \mathrm{~nm})$ causes an average field change of $\Delta H=0.9 \mathrm{~A} \cdot \mathrm{m}^{-1}$ in the case of a $4 \times 20 \mu \mathrm{m}$ sensor strip that is positioned $2 \mu \mathrm{m}$ beneath the magnetosome. The fact that this signal is higher than that of a considerably larger superparamagnetic bead suggests that ferro- or ferrimagnetic particles might be interesting candidates for magnetic reporters. However, the vast majority of commercially available magnetic labels are superparamagnetic, which reflects their use in situations (e.g., immunomagnetic separation systems) where it is desirable to be able to switch the magnetic interactions on and off by means of an external field. Any remnant magnetization in such cases would complicate the separation process. One commercially available type of ferromagnetic particles is used in magnetic twisting cytometry, ${ }^{24}$ and is available in 2.2 and $4.4 \mu \mathrm{m}$ sizes from Spherotech. The modeling of the $4.4 \mu \mathrm{m}$ particle shows that it should be easily detectable even with the present $4 \times 20 \mu \mathrm{m}$ sensor strips, with an average field deviation of $58 \mathrm{~A} \cdot \mathrm{m}^{-1}$ for a $4-\mu \mathrm{m}$ separation gap.

Calculations of expected average field signatures for various targets and sensor sizes are summarized in Tables 1 (sensor-target separation of $4 \mu \mathrm{m}$ ), and 2 (sensor-target separation of $2 \mu \mathrm{m})$. The results suggest that detection of individual magnetotactic bacteria or superparamagnetic beads might become feasible with $2 \times 2 \mu \mathrm{m}$ sensors, assuming a sensortarget separation of $2 \mu \mathrm{m}$. 
Table 1. Expected magnetic signatures from different targets, averaged for sensors of three sizes, for a sensortarget separation gap of $4 \mu \mathrm{m}$.

\begin{tabular}{|c|c|c|c|}
\hline Target & $4 \times 20 \mu \mathrm{m}$ sensor & $2 \times 10 \mu \mathrm{m}$ sensor & $2 \times 2 \mu \mathrm{m}$ sensor \\
\hline $\begin{array}{l}\text { Superparamagnetic } \\
\text { bead Dynal M270, } \\
2.8 \mu \mathrm{m}, \chi=0.17\end{array}$ & $0.162 \mathrm{~A} \cdot \mathrm{m}^{-1}$ & $0.422 \mathrm{~A} \cdot \mathrm{m}^{-1}$ & $0.627 \mathrm{~A} \cdot \mathrm{m}^{-1}$ \\
\hline $\begin{array}{l}\text { Magnetosome of a } \\
\text { magnetotactic } \\
\text { bacterium, } \\
0.04 \times 0.05 \times 1 \mu \mathrm{m} \text {, } \\
B_{r}=0.6 \mathrm{~T}\end{array}$ & $0.357 \mathrm{~A} \cdot \mathrm{m}^{-1}$ & $0.695 \mathrm{~A} \cdot \mathrm{m}^{-1}$ & $1.047 \mathrm{~A} \cdot \mathrm{m}^{-1}$ \\
\hline $\begin{array}{l}\text { Ferromagnetic bead } \\
\text { Spherotech, } 4.4 \mu \mathrm{m} \text {, } \\
B_{r}=0.01 \mathrm{~T}\end{array}$ & $58 \mathrm{~A} \cdot \mathrm{m}^{-1}$ & $91 \mathrm{~A} \cdot \mathrm{m}^{-1}$ & $114 \mathrm{~A} \cdot \mathrm{m}^{-1}$ \\
\hline
\end{tabular}

Table 2. Expected magnetic signatures from different targets, averaged for sensors of three sizes, for a sensortarget separation gap of $2 \mu \mathrm{m}$.

\begin{tabular}{|c|c|c|c|}
\hline Target & $4 \times 20 \mu \mathrm{m}$ sensor & $2 \times 10 \mu \mathrm{m}$ sensor & $2 \times 2 \mu \mathrm{m}$ sensor \\
\hline $\begin{array}{l}\text { Superparamagnetic } \\
\text { bead Dynal M270, } \\
2.8 \mu \mathrm{m}, \chi=0.17\end{array}$ & $0.636 \mathrm{~A} \cdot \mathrm{m}^{-1}$ & $1.607 \mathrm{~A} \cdot \mathrm{m}^{-1}$ & $2.819 \mathrm{~A} \cdot \mathrm{m}^{-1}$ \\
\hline $\begin{array}{l}\text { Magnetosome of a } \\
\text { magnetotactic } \\
\text { bacterium, } \\
0.04 \times 0.05 \times 1 \mu \mathrm{m} \text {, } \\
B_{r}=0.6 \mathrm{~T}\end{array}$ & $0.917 \mathrm{~A} \cdot \mathrm{m}^{-1}$ & $2.701 \mathrm{~A} \cdot \mathrm{m}^{-1}$ & $5.97 \mathrm{~A} \cdot \mathrm{m}^{-1}$ \\
\hline $\begin{array}{l}\text { Ferromagnetic bead } \\
\text { Spherotech, } 4.4 \mu \mathrm{m}, \\
B_{r}=0.01 \mathrm{~T}\end{array}$ & $119 \mathrm{~A} \cdot \mathrm{m}^{-1}$ & $225 \mathrm{~A} \cdot \mathrm{m}^{-1}$ & $332 \mathrm{~A} \cdot \mathrm{m}^{-1}$ \\
\hline
\end{tabular}

\section{Detection of ferromagnetic beads in flow}

In a proof-of-principle experiment, a suspension of premagnetized ferromagnetic beads was metered at the rate of $1.5 \mu \mathrm{L} / \mathrm{min}$ to the microfluidic GMR chip with $4 \times 20 \mu \mathrm{m}$ sensors. The data acquisition rate was set to $10 \mathrm{kHz}$. The obtained signals are shown in Figure 7 . 

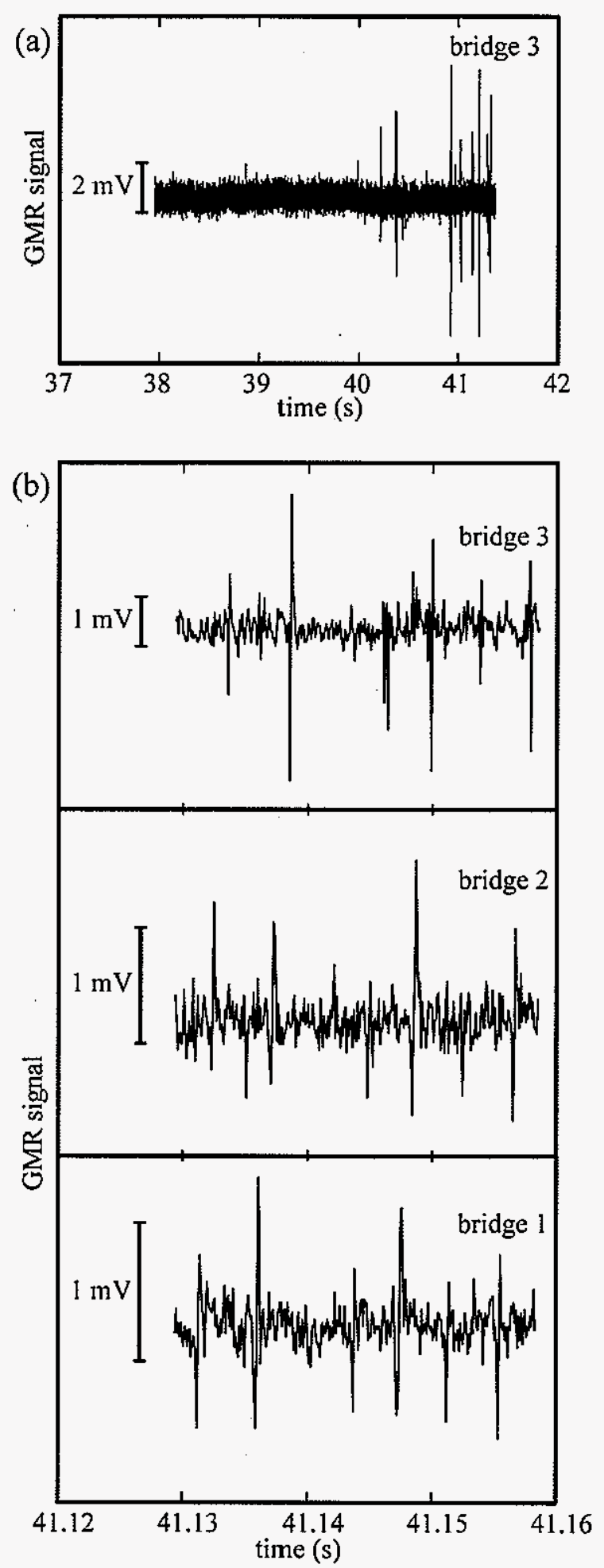

Figure 7. (a) GMR response of a sensor bridge (90x amplification, $796 \mathrm{~A} \cdot \mathrm{m}^{-1}$ applied field), showing a blank baseline signal followed by signals of a group of $4.4 \mu \mathrm{m}$ ferromagnetic beads. (b) A detail of the data from the same experiment, showing responses of all three sensor bridges. The raw signals were amplified (bridge 1 $29 \mathrm{x}$; bridge $2-34 \mathrm{x}$; bridge $3-90 \mathrm{x}$ ). 
The data in Figure 7(a) shows the baseline signal when only aqueous carrier was present in the channel, followed by a group of signal peaks from individual beads. Although particles interact via magnetic dipole forces, the channel width of only $13 \mu \mathrm{m}$ makes it highly unlikely that clusters of $4.4 \mu \mathrm{m}$ particles contribute to the signal. The fluidic shear forces and the presence of the surfactant further suppressed particle-particle interaction. A detail of the responses from the three bridges is shown in Figure 7(b), where the matching signal pattern, albeit with fluctuations in amplitudes, is evident in all three signals, and individual beads can be enumerated with reasonable confidence. To further substantiate that the signal pattern stems from the actual flow of beads, the time lag between the three signal channels was determined from the cross-correlation functions of squared derivatives of the three signals. Figure 8 shows the cross-correlation results, with the bridge 1-bridge 3 time lag being approximately twice as long as the lag between the bridge 1 and 2 , or 2 and 3 , therefore confirming that the observed pattern is due to the flow of magnetic beads, since the separation of the sensor bridges is $\Delta x_{1-2}=106 \mu \mathrm{m}$ and $\Delta x_{2-3}=100 \mu \mathrm{m}$.

The signal amplitude in Figure 7 is lower than that expected from the results given in Table 1 - the average field change of $58 \mathrm{~A} \cdot \mathrm{m}^{-1}(0.73 \mathrm{Oe})$ translates into the expected maximum signal of around $25 \mathrm{mV}$, based on the amplification gain of 90 (bridge 3), bias voltage $\mathrm{E}_{\text {bias }}=0.5 \mathrm{~V}$, and the sensor sensitivity of $0.08 \% / \mathrm{Oe}$. Possible reasons for this are 1) insufficient sampling rate compared to the bead velocities, and 2) hydrodynamic lift forces focusing the beads toward the channel central axis. Perhaps an even more important notion is that the relative amplitudes of the signals from individual beads differ for different sensor bridges. This finding suggests that magnetic moments of beads might be fluctuating due to the gyration induced by fluidic shear forces in a parabolic flow field present in microchannels. The shear-force induced torque in our system is opposed by the tendency of the bead to align its magnetic moment with the externally applied field. Further investigation of the interplay of shear forces and the magnetic torque is therefore warranted, as it not only addresses the practicalities of the magnetic flow cytometry, but also potentially opens new ways of probing and quantifying the intricacies of fluidic forces in particle-laden flows. 


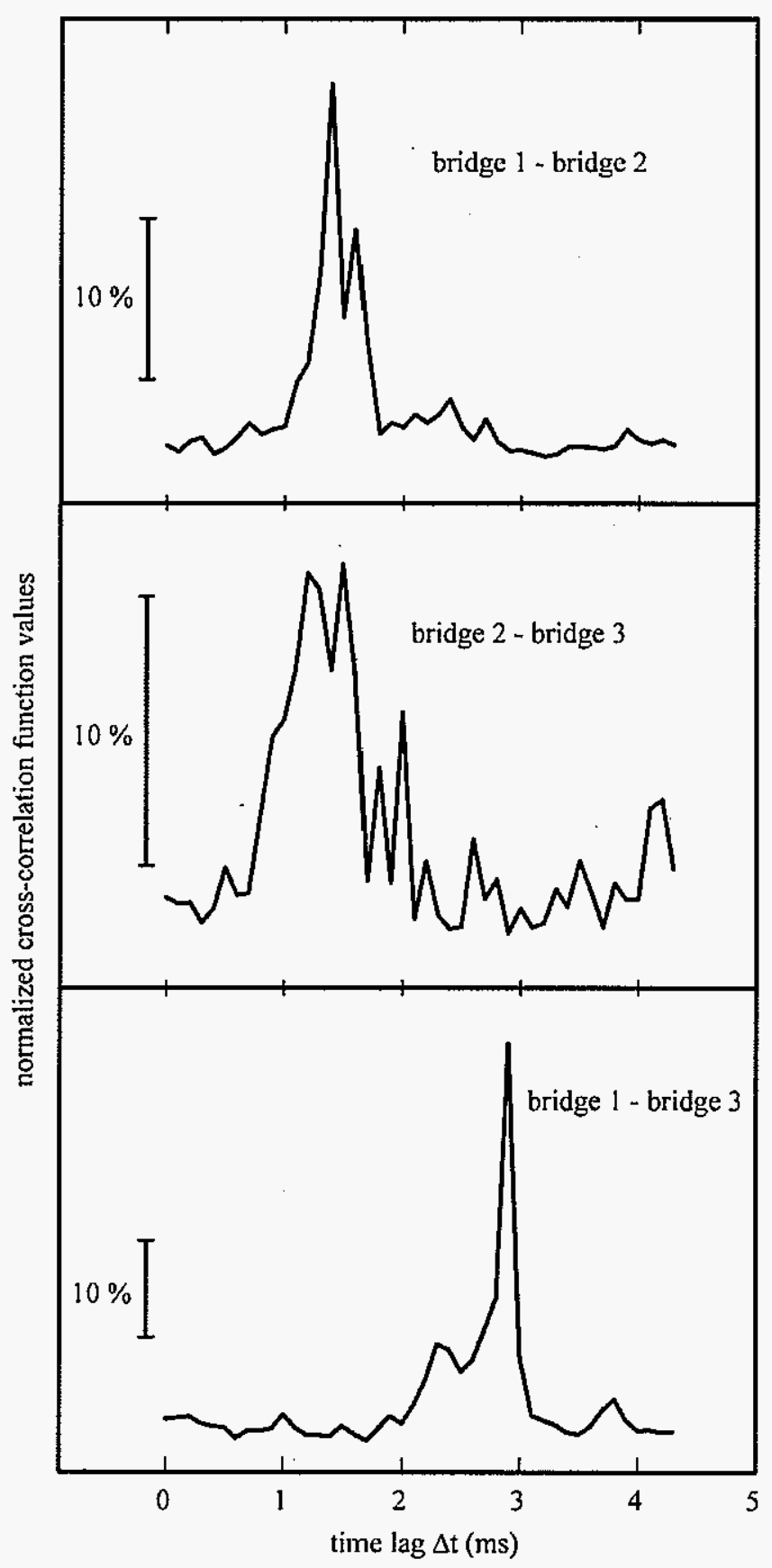

Figure 8. Normalized cross-correlation functions for the squared derivatives of signals shown in Figure 7.

\section{Conclusions}

Presented experimental and theoretical data demonstrate the viability of spin valves as integrated sensor elements for magnetic flow cytometry. The limit of detection has been determined in practical terms of the lowest detectable variation of the field, and compared to numerical models of representative targets of interest. This comparison suggests that the 
existing designs, although not optimized for single-particle detection, enables sensing of individual ferromagnetic microparticles in flow. This claim has been backed by experimental data. Furthermore, the comparison indicates that detection of magnetotactic bacteria or superparamagnetic labels requires redesigned sensors of a smaller size. This requirement, in turn, calls for a method for precise navigation of the sample stream through the detection volume above the sensor. These conclusions are currently being investigated in more detail and implemented in the design of a magnetic flow cytometer.

Direct and quantitative determination of magnetic material in microchannels might also be of interest in the area of on-chip synthesis of magnetic nanoparticles, ${ }^{25}$ where embedded magnetic sensors would enable continuous monitoring of the magnetic properties of the product. Finally, the amount of immunomagnetic labels binding to the cell surface has been shown to indicate the surface concentration of antigenic sites. ${ }^{26}$ Quantitative magnetic measurements can therefore provide valuable information about the levels of expression of certain receptors at the single-cell level.

\section{Acknowledgements}

Support from NSF's XYZ-on-a-Chip Initiative (\#88214), the W. M. Keck Foundation, the Institute for Combinatorial Discovery of Iowa State University, and DARPA's BioMagnetICs program is gratefully acknowledged. NP thanks ConocoPhillips for the support of a graduate fellowship. The Ames Laboratory is operated for the U.S. Department of Energy by Iowa State University under contract W-7405-eng-82.

\section{References cited}

1. Göttlinger, C., Mechtold, B. \& Radbruch, A. in Flow Cytometry and Cell Sorting (ed. Radbruch, A.) 3-25 (Springer-Verlag, Berlin, 2000).

2. Raybourne, R. \& Tortorello, M. in Detecting pathogens in food (ed. McMeekin, T. A.) (Woodhead Publishing, Cambridge, England, 2003).

3. Cheung, K., Gawad, S. \& Renaud, P. Impedance spectroscopy flow cytometry: on-chip label-free cell differentiation. Cytometry, Part A 65A, 124-132 (2005).

4. Chun, H., Chung, T. D. \& Kim, H. C. Cytometry and Velocimetry on a Microfluidic Chip Using Polyelectrolytic Salt Bridges. Analytical Chemistry 77, 2490-2495 (2005).

5. Wang, M. M. et al. Microfluidic sorting of mammalian cells by optical force switching. Nature Biotechnology 23, 83-87 (2005).

6. McClain, M. A., Culbertson, C. T., Jacobson, S. C. \& Ramsey, J. M. Flow cytometry of Escherichia coli on microfluidic devices. Analytical Chemistry 73, 5334-5338 (2001). 
7. Valer, M. Measuring multiple apoptosis parameters on a microfluidics chip-based instrument. American Biotechnology Laboratory 21, 28L-28N (2003).

8. Rife, J. C. et al. Design and performance of GMR sensors for the detection of magnetic microbeads in biosensors. Sensors and Actuators, A: Physical A107, 209-218 (2003).

9. Millen, R. L., Kawaguchi, T., Granger, M. C., Porter, M. D. \& Tondra, M. Giant Magnetoresistive Sensors and Superparamagnetic Nanoparticles: A Chip-Scale Detection Strategy for Immunosorbent Assays. Analytical Chemistry 77, 6581-6587 (2005).

10. Li, G. et al. Spin valve sensors for ultrasensitive detection of superparamagnetic nanoparticles for biological applications. Sensors and Actuators, A: Physical A126, 98106 (2006).

11. Molday, R. S., Yen, S. P. \& Rembaum, A. Application of magnetic microspheres in labelling and separation of cells. Nature (London) 268, 437-8 (1977).

12. Miltenyi, S., Muller, W., Weichel, W. \& Radbruch, A. High gradient magnetic cell separation with MACS. Cytometry 11, 231-8 (1990).

13. $\mathrm{Hu}, \mathrm{J}$. et al. Improvement of recoveries for the determination of protozoa Cryptosporidium and Giardia in water using method 1623. Journal of Microbiological Methods 58, 321-325 (2004).

14. Bazylinski, D. A. \& Frankel, R. B. Magnetosome formation in prokaryotes. Nature Reviews Microbiology 2, 217-230 (2004).

15. Tondra, M., Porter, M. \& Lipert, R. J. Model for detection of immobilized superparamagnetic nanosphere assay labels using giant magnetoresistive sensors. Journal of Vacuum Science \& Technology, A: Vacuum, Surfaces, and Films 18, 1125-1129 (2000).

16. Pekas, N., Porter, M. D., Tondra, M., Popple, A. \& Jander, A. Giant magnetoresistance monitoring of magnetic picodroplets in an integrated microfluidic system. Applied Physics Letters 85, 4783-4785 (2004).

17. Thorsen, T., Roberts, R. W., Arnold, F. H. \& Quake, S. R. Dynamic Pattern Formation in a Vesicle-Generating Microfluidic Device. Physical Review Letters 86, 4163-4166 (2001).

18. Link, D. R., Anna, S. L., Weitz, D. A. \& Stone, H. A. Geometrically mediated breakup of drops in microfluidic devices. Physical review letters 92, 054503 (2004).

19. Zheng, B., Roach, L. S. \& Ismagilov, R. F. Screening of protein crystallization conditions on a microfluidic chip using nanoliter-size droplets. Journal of the American Chemical Society 125, 11170-11171 (2003).

20. Dunin-Borkowski, R. E. et al. Off-axis electron holography of magnetotactic bacteria: magnetic microstructure of strains MV-1 and MS-1. European Journal of Mineralogy 13, 671-684 (2001). 
21. Wang, D. in Handbook of Advanced Magnetic Materials (eds. Liu, Y., Sellmyer, D. J. \& Shindo, D.) 307-338 (2006).

22. Tondra, M. Personal Communication, June 27, 2006.

23. Helseth, L. E., Fischer, T. M., Hansen, R. W. \& Johansen, T. H. Microscopic magnetic squeezer. Applied Physics Letters 85, 2556-2558 (2004).

24. Butler, J. P. \& Kelly, S. M. A model for cytoplasmic rheology consistent with magnetic twisting cytometry. Biorheology 35, 193-209 (1998).

25. Song, Y. et al. Microfluidic Synthesis of Cobalt Nanoparticles. Chemistry of Materials 18, 2817-2827 (2006).

26. Leigh, D. R., Steinert, S., Moore, L. R., Chalmers, J. J. \& Zborowski, M. Cell tracking velocimetry as a tool for defining saturation binding of magnetically conjugated antibodies. Cytometry, Part A 66A, 103-108 (2005). 


\title{
CHAPTER 5. TOWARD MAGNETIC FLOW CYTOMETRY ON A CHIP: DETECTION OF MAGNETOTACTIC BACTERIA IN A MICROFLUIDIC SYSTEM WITH EMBEDDED MAGNETORESISTIVE SENSORS
}

\author{
A paper to be submitted to Analytical Chemistry \\ Nikola Pekas and Marc D. Porter \\ Institute for Combinatorial Discovery, Departments of Chemistry and Chemical \\ Engineering, and Ames Laboratory-USDOE, lowa State University, Ames, Iowa 50011 \\ Dennis Bazylinski \\ Department of Biochemistry, Biophysics, and Molecular Biology, \\ Iowa State University, Ames, Iowa 50011 \\ Mark Tondra, Anthony Popple, and Albrecht Jander \\ NVE Corporation, Eden Prairie, Minnesota 55433
}

\begin{abstract}
We describe the design, fabrication, and evaluation of a microfabricated, magneticsbased flow cytometer. The device uses giant magnetoresistance (GMR) technology as cornerstone to realize rapid detection of magnetic targets in flow. GMR sensors are $2 \times 2 \mu \mathrm{m}$ in size, and are embedded into the bottom of the flow channel. The microfluidic layout of the device incorporates a novel three-dimensional fluidic focusing scheme that is easy to accomplish through two simple; standard fabrication steps. Preliminary experimental evaluation using magnetotactic bacteria as model target suggests the single-cell detection capability, and demonstrates the viability of the magnetic detection concept in the context of lab-on-a-chip platforms.
\end{abstract}

\section{Introduction}

Earlier reports from our laboratory have described a set of microfabricated tools for magnetic manipulation and detection, demonstrated their functionality, and assessed the general requirements for detection of magnetic targets in flow. Here we extend that work to design, fabricate, and evaluate a magnetics-based flow cytometer. Preliminary experimental 
work is based on giant magnetoresistance (GMR) spin-valve sensors and magnetotactic bacteria (MTB) as the target, but has broader implications to a general case of magnetically labeled cells.

GMR sensors are microfabricated arrangements of alternating layers of magnetic and nonmagnetic materials that exhibit a large change in resistance in the presence of an external magnetic field. ${ }^{1,2}$ Moreover, the footprint of a GMR sensor can be exceedingly small, which, when coupled with the technological landscape already in place pertaining to their use as high speed, high density data readout devices, presents an intriguing opportunity for the creation of "point of use" detection systems. 3 .7

MTB represent a heterogeneous group of motile prokaryotes that synthesize intracellular iron oxide or iron sulfide ferrimagnetic nanoparticles. ${ }^{8,9}$ They are ubiquitous in sediments of freshwater and marine habitats, and in chemically stratified water columns. The highest numbers, sometimes up to $30 \%$ of the total microbial volume, are found at the oxic-anoxic transition zone. Consequently, all known MTB species are found to be either microaerophilic or anaerobic, and exhibit a negative tactic response to atmospheric concentrations of oxygen (i.e., negative aerotaxis). The intracellular presence of highly ordered linear chains of singledomain magnetic nanoparticles enables these microorganisms to passively align with the geomagnetic or other magnetic fields, a behavior labeled as magnetotaxis. This mechanism is combined with flagellar motility, chemotaxis, and aerotaxis to define a complex lifestyle adapted for vertical chemical and redox gradients. As a result, only a small fraction of naturally occurring MTB species has been isolated in pure cultures. An illustration of the passive response of MTB cells to external magnetic field $\left(\mathrm{H}_{\text {app }}\right)$ is given in Figure 1.
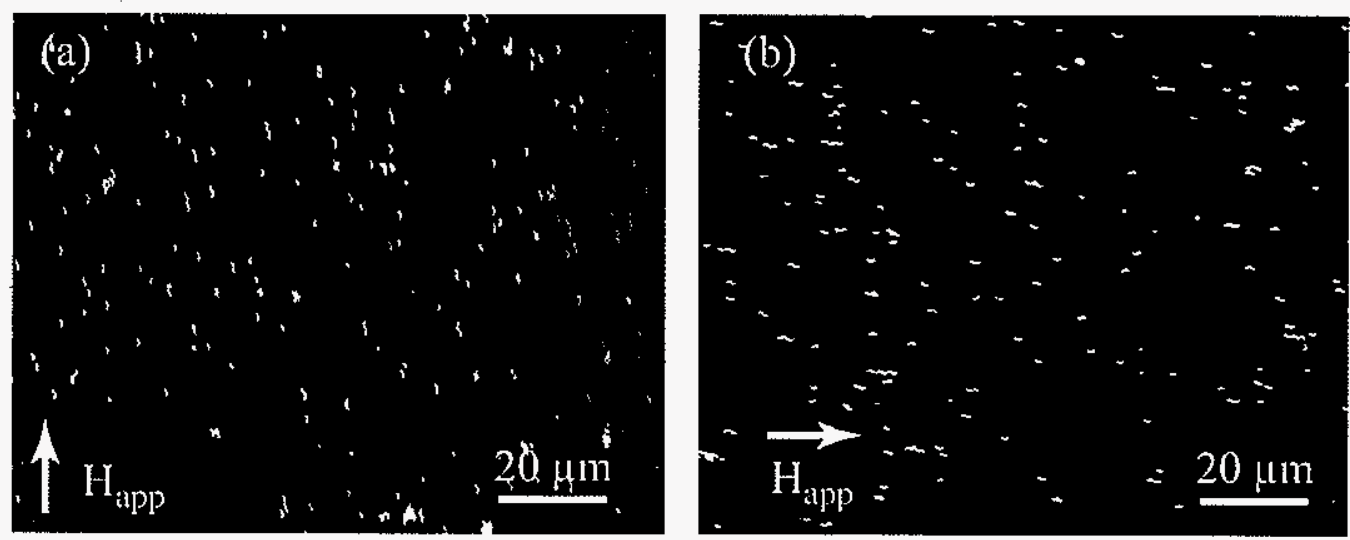

Figure 1. Fluorescence micrographs of AMB-1 magnetotactic cells in a uniform magnetic field oriented vertically (a), and in a uniform horizontal field (b). The cells were treated with glutaraldehyde, stained with Syto-64 DNA stain (Invitrogen), and imaged through a 40x objective lens. The field was applied by two miniature rare-earth magnets. 
The interest in MTB ranges from nanotechnology ${ }^{10}$ to biotechnology ${ }^{11}$ and ecology. ${ }^{12}$ The detection of MTB is commonly realized by magnetic enrichment of the cells followed by the microscopic identification. One example of a less common approach was described by Chemla et al., and involves the use of a SQUID microscope to assess the motility of single MTB cells. ${ }^{13}$ We believe that a microminiaturized magnetic flow cytometer based on giant magnetoresistive sensors would be valuable in several ways: 1) as a field deployable apparatus for detection and enumeration of MTB as ecological indicators; 2) as an enabling device for in vitro single-cell assessment of the motility and the magnetic status in MTB populations; and 3) in screening for the appropriate conditions to isolate pure MTB cultures. Finally, such devices, in conjunction with magnetic labeling procedures, would be important as a general cytometry tool with a broad set of applications in biomedical diagnostics, drug discovery, and food safety.

\section{Design considerations}

Two key issues were identified in a recent report that critically examined the design and performance challenges in the development of a GMR flow cytometer. First, the sensor size needs to be in the range of several micrometers to make single cell detection possible. Moreover, the proximity of the target to sensor is crucial, since observed field decays with the third power of distance. ${ }^{14,15}$ These requirements necessitate precise navigation of the sample stream over sensors. Simply pumping the sample through channels with small crosssections can pose two problems: 1) clogging in the presence of particles with a distribution of sizes or particle aggregates, and 2) fabrication techniques become more challenging with downscaling the feature sizes. We therefore choose to develop a detection format where micrometer-sized GMR sensors are buried beneath the channel of a relatively large crosssection. Upstream from sensors, the sample stream is hydrodynamically focused by a buffer flow from the sides and from top to form a narrow and thin flow stream along the central bottom portion of the channel (see Figure 2). The sense elements in sensor bridges are centered along the projection of the sample stream after completing both focusing steps. 


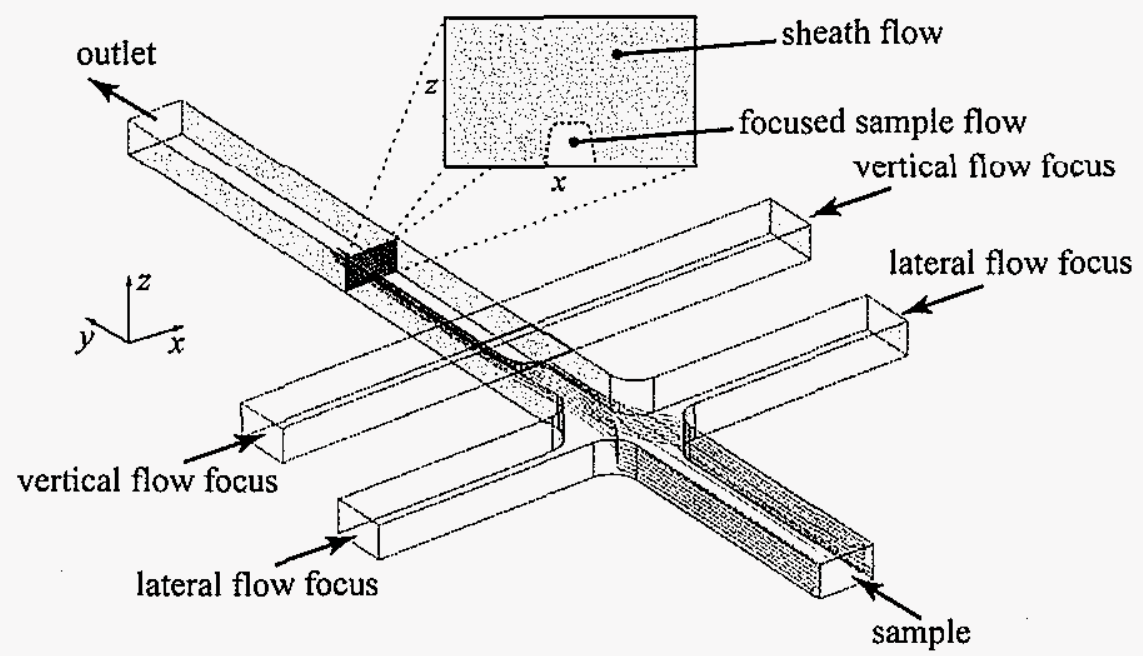

Figure 2. A conceptual drawing of the three-dimensional focusing scheme. The sample stream is first narrowed in a "pinch-flow" configuration, and then confined towards the channel bottom by a vertical-focus stream that enters from the top of of sample flow channel. Streamlines are shown to visualize the change of the sample stream boundaries.

Since sample flow is confined and focused by surrounding and collapsing the sample stream with fluid rather then solid constrictions, this approach greatly reduces the risk of channel clogging. To ensure that target particles traverse precisely the sensor field of view, the flow rates from the two branches of the lateral-focus system need to be carefully balanced. This is illustrated in Figure 3, which shows the numerical modeling results of the system described above, and compares the sample-stream profiles for a balanced and an imbalanced ( $\pm 10 \%$ deviation) lateral focus flow. The models were constructed in three dimensions using FEMLAB package (Comsol), in the incompressible Navier-Stokes mode. The viscosity was set to $0.9 \mathrm{mPa} \cdot \mathrm{s}$, and the sample stream boundaries were determined by tracking the streamlines that started at points adjacent to the side walls and the top of the sample inlet. 


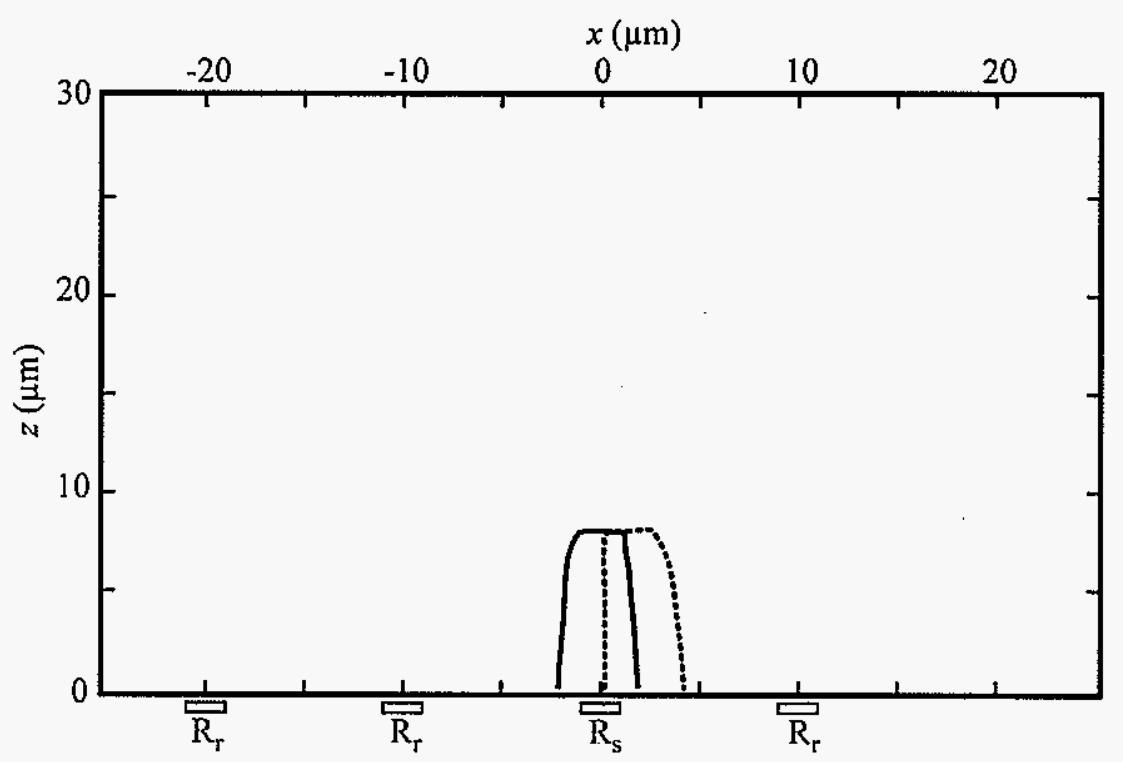

Figure 3. Cross-sectional views of a three-dimensional numerical model of the system shown in Figure 2. The plot shows the sample stream profile (solid line) in the $x-z$ plane $100 \mu \mathrm{m}$ downstream from the vertical focusing junction, for the following set of parameters: sample flow rate $\omega_{s}=0.3 \mu \mathrm{L} / \mathrm{min}$, total lateral focus flow rate $\omega_{t}=$ $\omega_{h, \text { left }}+\omega_{l, \text { right }}=3.6 \mu \mathrm{L} / \mathrm{min}$, and total vertical focus flow rate $\omega_{\nu}=\omega_{1, \text { lefs }}+\omega_{1, \text { right }}=13.5 \mu \mathrm{L} / \mathrm{min}$. The dotted line depicts the sample stream profile when the two lateral flow rates differ by $10 \%$ from the balanced value: $\omega_{1, \text { left }}=$ $1.98 \mu \mathrm{L} / \mathrm{min}$ and $\omega_{i, r i g h t}=1.62 \mu \mathrm{L} / \mathrm{min}$. Also shown are the positions of four Wheatstone bridge elements: a sense $\left(R_{S}\right)$ and three reference $\left(R_{r}\right)$ spin valves. The layout of the bridges is shown in Figure 4.

A similar model for the case of imbalanced vertical focus flow rates ( $\pm 10 \%$ deviation) shows only a slight drift of the sample stream from the middle of the channel $(x=0)$. To achieve balanced flow rates and to reduce the number of fluidic connections to the chip, the lateral and the vertical focus feed from two external pumps are each split into two streams on-chip, immediately following the inlet ports. A careful design of the overall fluidic structure, including channel constrictions that serve as fluidic ballast resistors, ensures symmetrical backpressures and therefore symmetrical flows in the focusing branches. Figure 4 gives an overview of two fluidic designs, and examples of two different sensor arrangements. 

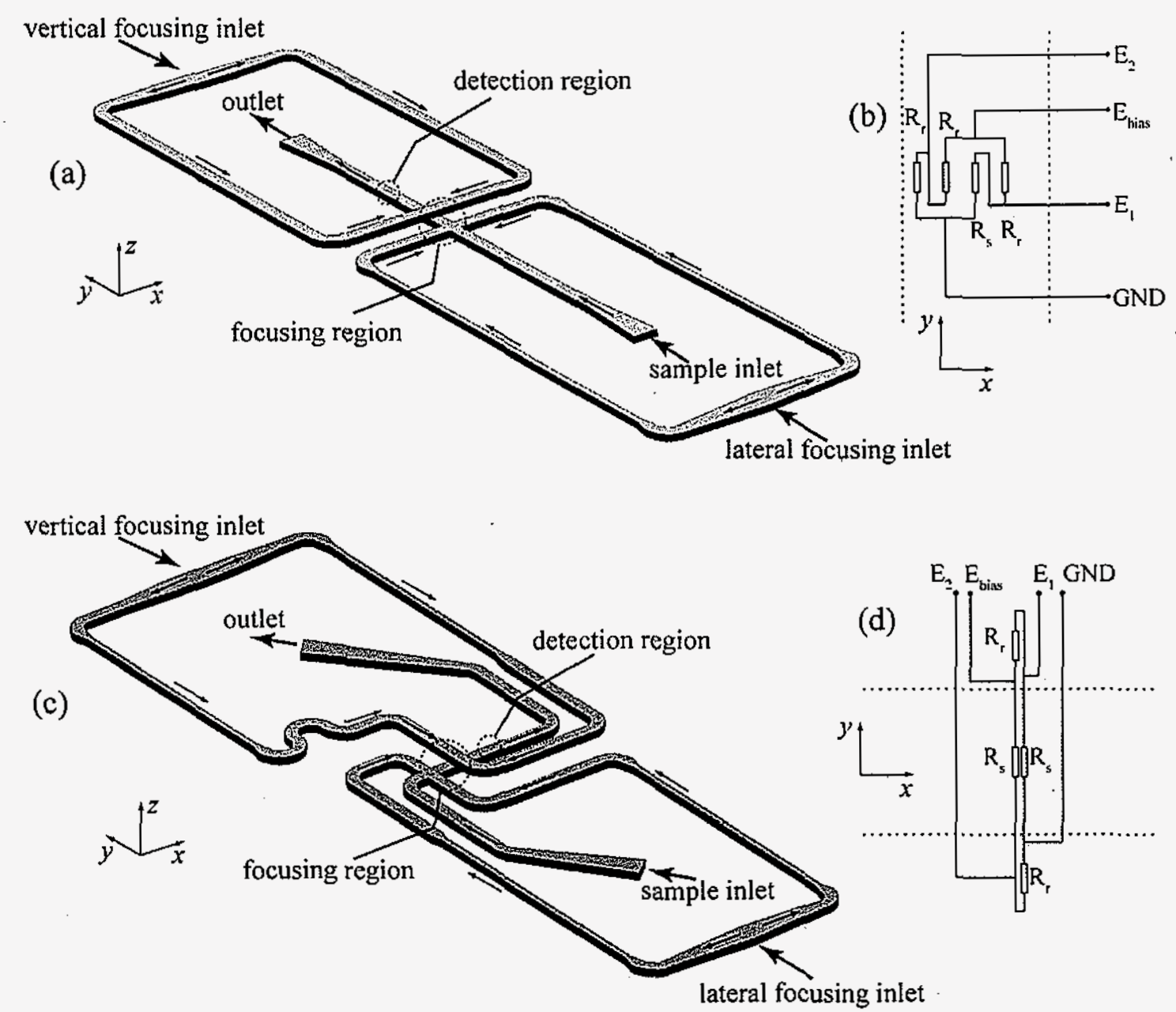

Figure 4. Fluidic layouts with corresponding schematics of Wheatstone bridges. The two layouts differ in direction of the sample flow relative to external field. An external field is in all cases applied along the $x$-axis. (a) The sample flow through the detection region is oriented along the $y$-axis and perpendicular to the applied field. (b) A schematic diagram of the Wheatstone bridge featuring a single sense element $\left(R_{s}\right)$ and three reference elements $\left(R_{r}\right)$. The channel walls are represented by dotted lines. (c) The sample flow through the detection region is parallel to the $x$-axis and to the applied field. (d) A Wheatstone bridge featuring a pair of sense elements $\left(R_{s}\right)$ and a pair of references $\left(R_{r}\right)$.

Our systems utilize spin-valve GMR sensors ${ }^{5,16}$ that are fabricated as 30- $\mu \mathrm{m}$ long, $2-\mu \mathrm{m}$ wide strips, and they are sensitive to the transverse component of the magnetic field ( $x$-axis direction). The electrically active area that generates the electrical signal is defined by the positioning of the electrical contacts on the sensor, and is set to either $2 \times 2$ or $2 \times 10 \mu \mathrm{m}$ in our present design to accommodate for targets of different sizes. The sensors are arranged in a Wheatstone bridge configuration. ${ }^{7}$ In the case of one sense resistor $\left(R_{s}\right)$ and three reference resistors $\left(R_{r}\right)$, as in Figure 4(b), the measured signal is given by Equation 1: 


$$
E_{1}-E_{2}=\frac{R_{s}-R_{r}}{2\left(R_{s}+R_{r}\right)} E_{b i a s}
$$

where $E_{\text {bias }}$ is the constant bias voltage supplied to the bridge. In case of a pair of adjacent sense resistors, as in Figure 4(d), assuming that the fringe field of the target is uniform across the overall sense resistor area, the measured signal will be twice as large as that obtained from Equation 1. The choice of the electrical layout and sensor size will depend on the size of the target, and will also dictate the choice of the fluidic layouts [Figure 4 (a) and (c)].

Next, we explore how sample stream dimensions scale with the sample-to-focus flow rate ratios. In some cases, a simple linear approximation provides acceptable results. ${ }^{17}$ Hofmann et al. briefly discussed the experimentally observed non-linear scaling of sample thickness in vertical confinement flows. ${ }^{18}$ In the following discussion, we will give a more thorough analysis that reveals important phenomena that somewhat contradict day-to-day linear intuition. The non-linear behavior is a consequence of the coupling of mass conservation requirements with the parabolic flow velocity profiles present in microchannels. The finiteelement models were developed in FEMLAB based on fluidic layouts described above, and will allow us to estimate the experimental parameters - flow rates, target concentration range and the velocity distribution, and requisite sampling rates. Figure 5 shows results from twodimensional models of the lateral and vertical focusing junctions.

We define a relative flow rate as a ratio of the volumetric flow rate of the sample stream to the total volumetric flow rate. The models show that in case of the symmetrical "pinchflow" focusing [Figure 5 (a)], the sample stream width follows a linear correlation with the relative sample flow rate, at least up to a relative flow ratio of 0.33 . The sample-stream width, however, is narrower than that predicted by the simple rationing of flow rates, as the focus streams widen in order to compensate for their lower flow velocities in the parabolic flow profile. In case of the vertical confinement flow [Figure 5 (b)], a non-linear dependence is readily apparent, and arises because the sample stream confined to the vicinity of the channel bottom compensates for the lower flow velocity by forming a thicker layer. This situation means that the actual sample-stream thickness will always be higher than that predicted by simple rationing. These results can be used to estimate the relative flow rates required to achieve a given size of the sample stream. For example, in case of $2 \times 10 \mu \mathrm{m}$ sensors, with flow parallel to the field ( $x$-axis direction), the sample stream width should be around $10 \mu \mathrm{m}$, i.e., a $\Delta y$ of 0.2 , relative to the channel width of $50 \mu \mathrm{m}$. This width value translates into the relative sample flow rate of around 0.3 , or $\omega_{s} /\left(\omega_{s}+\omega_{l}\right)=0.3$, calling for the lateral focus flow rate of $\omega_{l}=2.3 \omega_{s}$. In a similar way, to achieve a thickness of at least 3 
$\mu \mathrm{m}$, or $\Delta z=0.1$ relative to the channel depth, the required vertical-focus flow rate is $\omega_{\mathrm{v}}=$ $107 \omega_{s}$, yielding a total flow rate of $\omega_{\text {total }}=110 \omega_{s}$. To understand the significance of the parabolic flow profiles in non-axisymmetric flow focusing systems, it is useful to compare the above factor of 110 to the ratio of the channel cross-section to the focused stream crosssection, which equals 50 in this example.
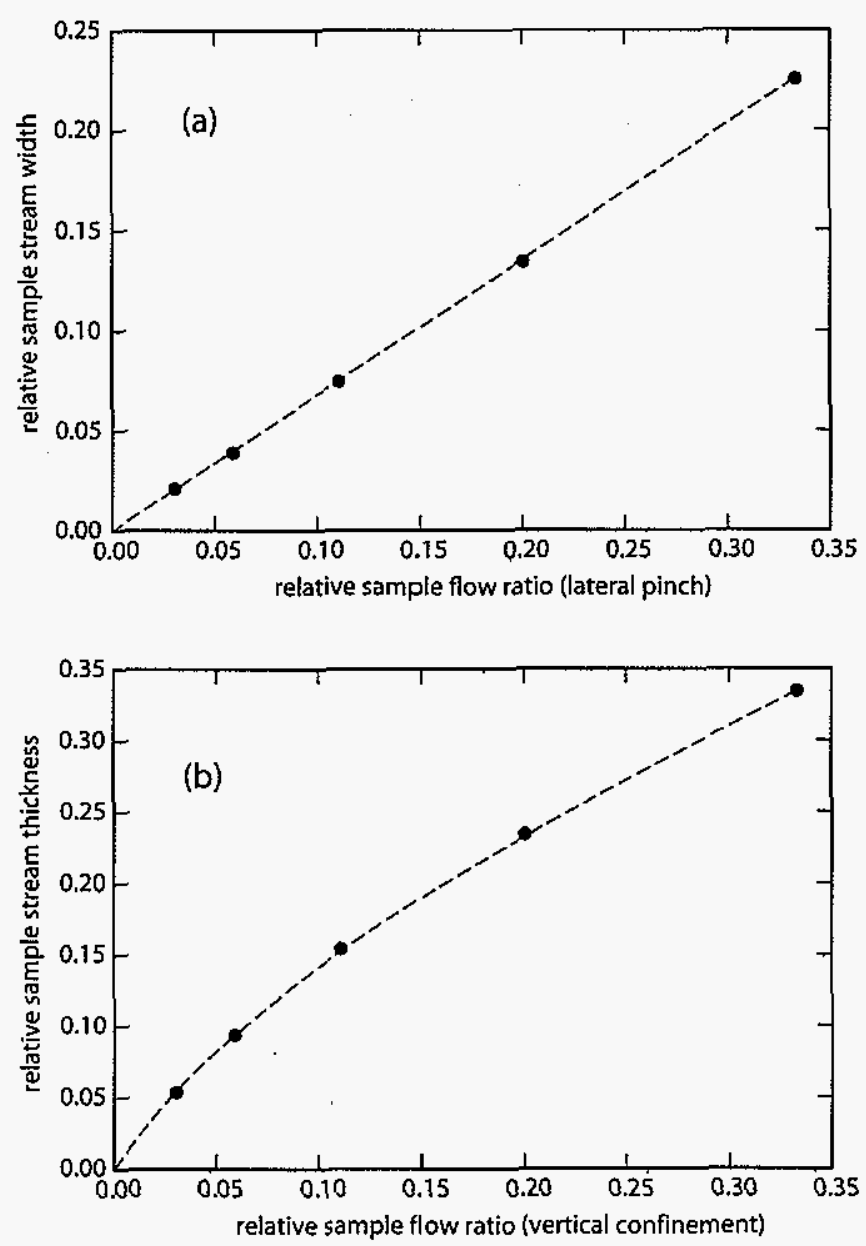

Figure 5.5. Compiled results from two-dimensional numerical modeling of the flow focusing architectures. Dashed lines are added as a guide to the eye. (a) Sample stream width relative to the $50-\mu \mathrm{m}$ wide channel versus sample flow rate relative to the total flow rate in a symmetrical lateral focusing configuration. (b) Sample stream thickness relative to the $30-\mu \mathrm{m}$ deep channel versus sample flow rate relative to the total flow rate in a vertical flow confinement configuration.

Further analysis of three-dimensional models reveals another non-linear effect (Figure 6) - a distortion of the sample streamlines upon focusing. The outcome is interesting and worth noting, since it suggests that an initially homogeneous suspension of target particles will tend to exhibit a slightly higher concentration in the top region of the focused sample stream. 


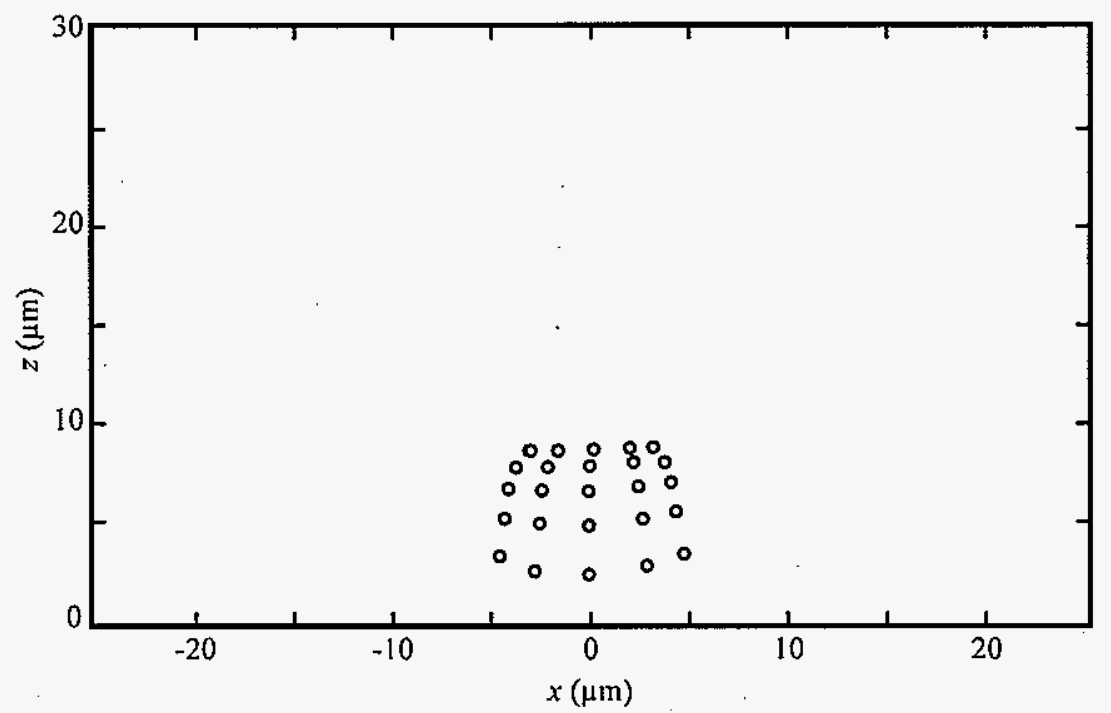

Figure 6. An initially uniform rectangular array of the sample streamlines maps non-uniformly into a focused sample stream. The initial array upstream from the focusing junctions consisted of $5 \times 6$ streamlines and covered the sample inlet cross-section. Upon focusing, the streamline array is distorted, with the streamline density higher in the top region than the bottom. The last row of streamlines is not shown since the flow in this region becomes significantly slow, making the integration time prohibitively long for our computation platform. The flow parameters are: sample flow rate $\omega_{s}=0.9 \mu \mathrm{L} / \mathrm{min}$, total lateral focus flow rate $\omega_{t}=\omega_{\text {, left }}+\omega_{\text {, right }}=3.6$ $\mu \mathrm{L} / \mathrm{min}$, and total vertical focus flow rate $\omega_{\nu}=\omega_{1, \text { left }}+\omega_{\nu, \text { right }}=13.5 \mu \mathrm{L} / \mathrm{min}$. Random fluctuations in streamline projections are a modeling artifact of the finite mesh element size.

\section{Fabrication}

In our earlier designs, the sensor strips extended beyond the channel walls, and electrical connections to $\sim 1-\mu \mathrm{m}$ thick metal lines were therefore protected from solution by being embedded in the BCB polymer. ${ }^{7}$ Since we are now deploying much smaller sensors, it is necessary to redesign the approach because introduction of the metal lines into the channel may cause topographical and electrical problems. The presence of metal interconnects will disrupt the flow profile and potentially limit access of target particles to the detection volume. Perhaps an even greater challenge arises from the need to electrically insulate the detection system. With smooth surfaces, a reliable passivation layer can be formed by sputtering a thin layer of silicon nitride. The presence of topographical features, however, compromises the quality of the passivation layer, particularly along the feature edges. To address this issue, a process was developed wherein spin-valves are patterned on top of a polished substrate with entrenched metal interconnects. The only topographical feature on the channel bottom is therefore the GMR strip which is only tens of nanometers in thick, and is therefore much easier to fully passivate with a $\sim 300 \mathrm{~nm}$ silicon nitride layer. A cross-section of the integrated microfluidic GMR sensing platform is shown in Figure 7. 


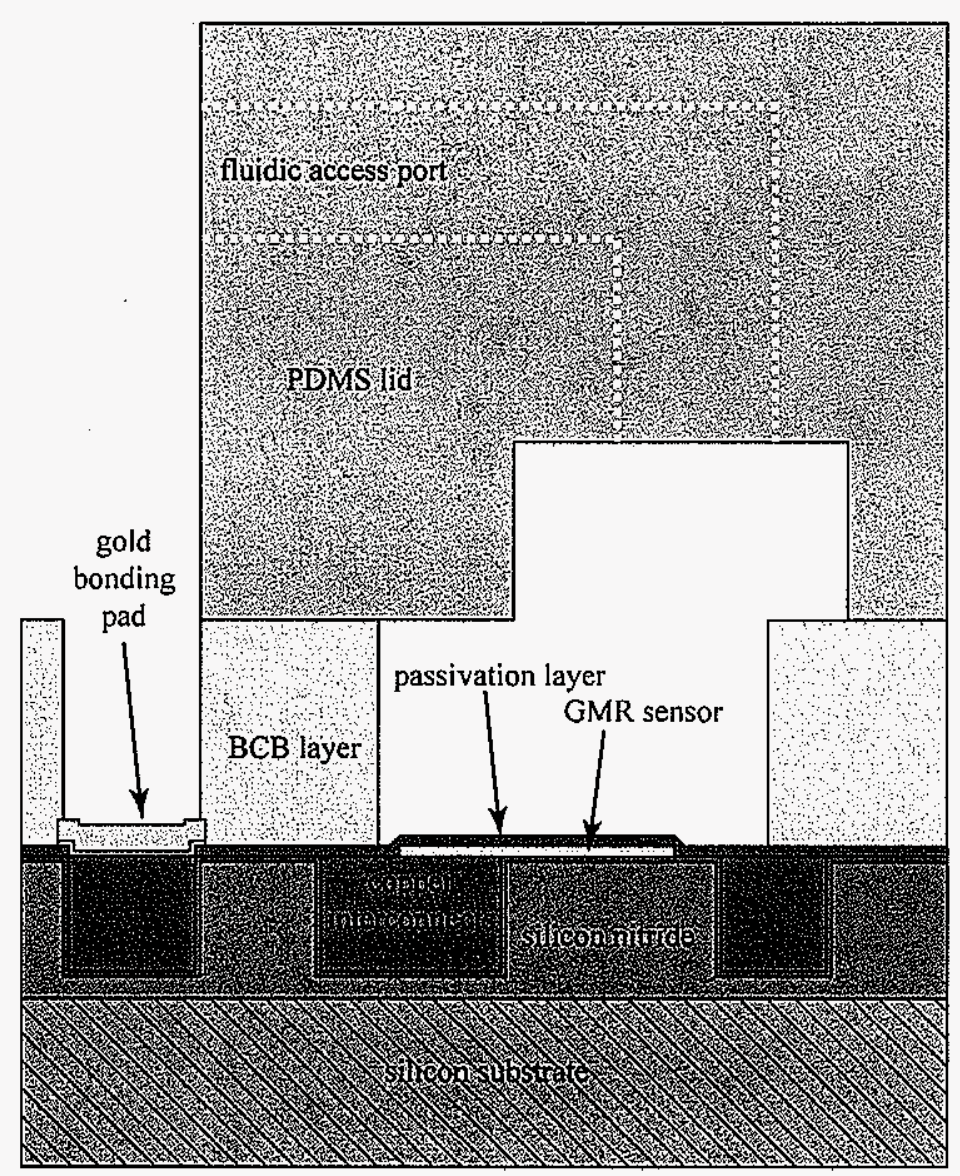

Figure 7. Process cross-section (not to scale). Electronic interconnections are realized in form of copper lines embedded in 1- $\mu \mathrm{m}$ silicon nitride. The GMR stack is deposited and protected by approximately $300 \mathrm{~nm}$ of sputtered silicon nitride. Gold is deposited and patterned to form bonding pads for electronic interfacing, and 35 $\mu \mathrm{m}$ layer of benzocyclobutene (BCB) is spun coated and patterned to define the lower level of fluidics. Also shown is a poly(dimethylsyloxane) (PDMS) lid with a channel defining the upper layer of fluidics and an Lshaped access port for fluidic interfacing.

The lids with microchannels and access ports were fabricated by micromolding as described in Chapters 2 and 3, with a difference that port-defining mold inserts were downsized from 360 to $280 \mu \mathrm{m}$ in diameter to accommodate four fluidic connections per chip. The L-shaped inserts were made from soft-annealed aluminum wire (Al 1199 from California Fine Wire), and carefully affixed to the predefined spots on the mold surface using a wire bonder (Hybond 572) with a modified electromechanical clamp assembly. Bonding of the lids was performed in the same fashion as described in previous Chapters. Upon the activation of the surfaces of a chip and the lid in oxygen plasma, the two pieces were aligned and brought into conformal contact by using the contact mask aligner. 


\section{Device evaluation}

Prior to bonding of the lids, the quality of bare devices was investigated. To evaluate the channel dimensions and profiles, a prepolymer mixture of PDMS was cast against one of the chips, degassed under house vacuum for one hour, and cured overnight at $60^{\circ} \mathrm{C}$. The cured PDMS was then peeled off the chip, and cut through to reveal the imprint cross section. Microscopical investigation (Figure 8) shows channels of a roughly trapezoidal shape, slightly overetched with the width approximately $15 \%$ larger than the anticipated values. The channel depth was measured to be about $35 \mu \mathrm{m}$.

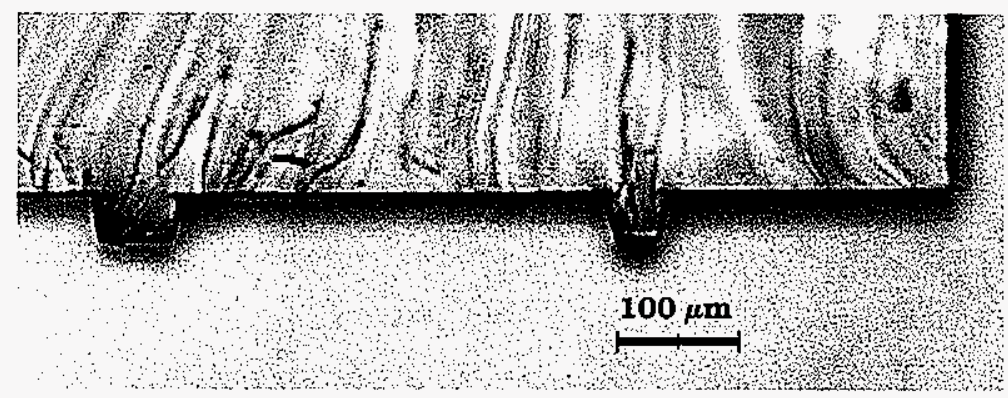

Figure 8. A micrograph of a section of the PDMS replica showing imprints of the sample channel and of one of the two lateral-focus channels. Apparent channel profiles are slightly trapezoidal, and channel widths are approx. $15 \%$ larger than the nominal widths in mask design.

Upon bonding the PDMS lids, devices were wire bonded to flexible printed-circuit boards and electronically tested by NVE Corporation. The magnetoresistance measurements yield an average MR ratio between 1 and $2 \%$ - a slightly worse performance than for the sensors described in previous Chapters, where the MR ratio averaged at around $4 \%$.

Assembled and packaged devices were investigated further (Figure 9). Delamination of the $\mathrm{BCB}$ layer is apparent from the interference patterns, particularly around corners however, the PDMS replicas did not reveal any significant undercuts. The BCB fluidic layer appears to be misaligned from the sensors, with approximate deviations of $\Delta x=2 \mu \mathrm{m}$ and $\Delta y=1 \mu \mathrm{m}$. 


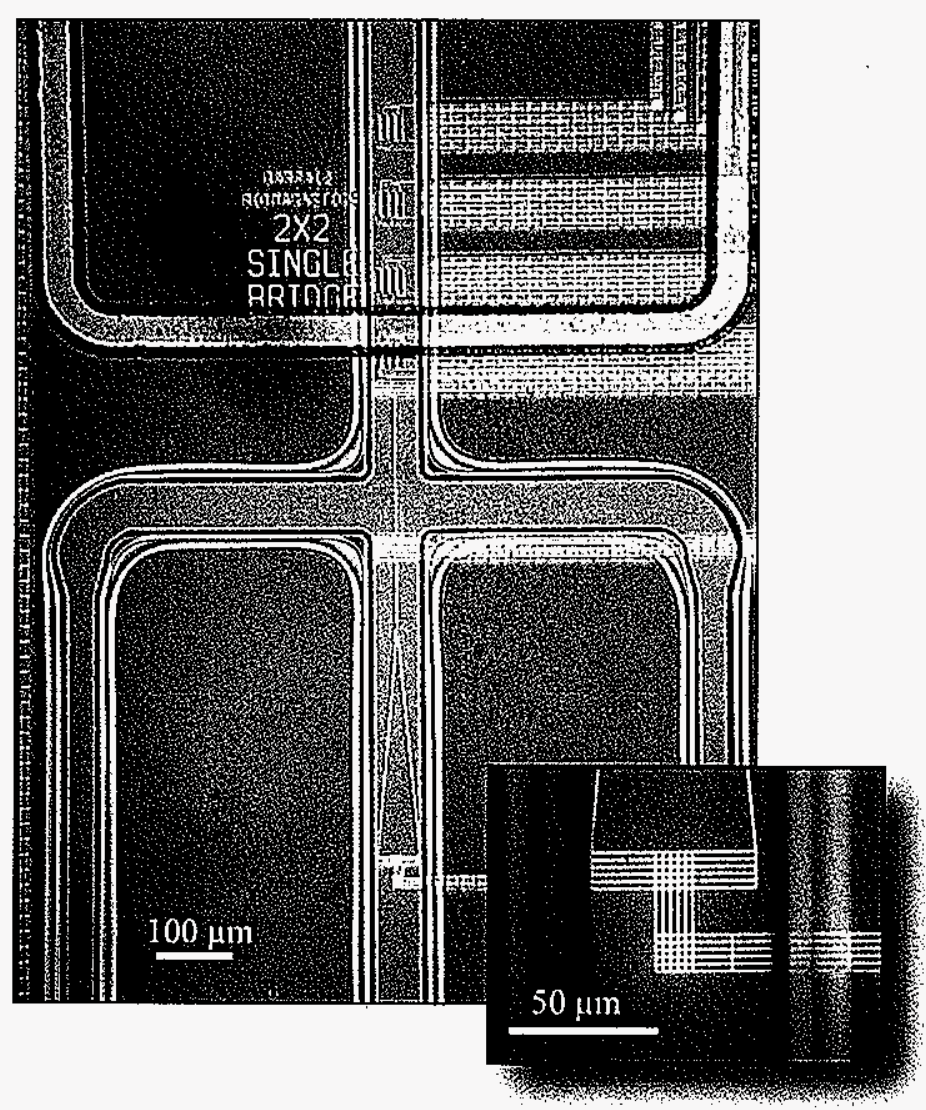

Figure 9. Top-view micrograph of a central portion of the assembled cytometer. The inset shows the detail where a slight misalignment of the fluidic and the electronic layers is noticeable - the metal structure is supposed to symmetrically span the channel width.

\section{Experiments}

\section{Determination of optimal volumetric flow rates}

Since the present sampling rate of acquisition electronics is limited to $100 \mathrm{kHz}$, we will use that figure a starting point to determine the optimal flow rates. In order to ensure reliable detection, we choose to have a minimum of 10 data points per individual target. For a $2-\mu \mathrm{m}$ sensor, the target flow-by velocity needs to be below $v_{\max }=2 \mu \mathrm{m} /(10 \times 100 \mathrm{kHz})=20 \mathrm{~mm} / \mathrm{s}$. The total flow rate through the detection channel needs to be low enough to ensure that the fastest moving target particles ( $3 \mu \mathrm{m}$ from the bottom) have a velocity below the $20 \mathrm{~mm} / \mathrm{s}$ limit. Based on the actual channel dimensions (trapezoidal cross section, $55 \mu \mathrm{m}$ bottom, 65 $\mu \mathrm{m}$ top, $35 \mu \mathrm{m}$ height), we calculated the flow velocity distribution (Figure 10) for different total flow rates, and estimated that the $20 \mathrm{~mm} / \mathrm{s}$ isosurface will be within $3 \mu \mathrm{m}$ from the channel bottom when the total volumetric flow rate is below $\omega_{\text {max, total }}=4.4 \mu \mathrm{L} / \mathrm{min}$. As explained earlier, the flow rates for the sample and focus feeds can be calculated from this value for a desired sample stream width. 


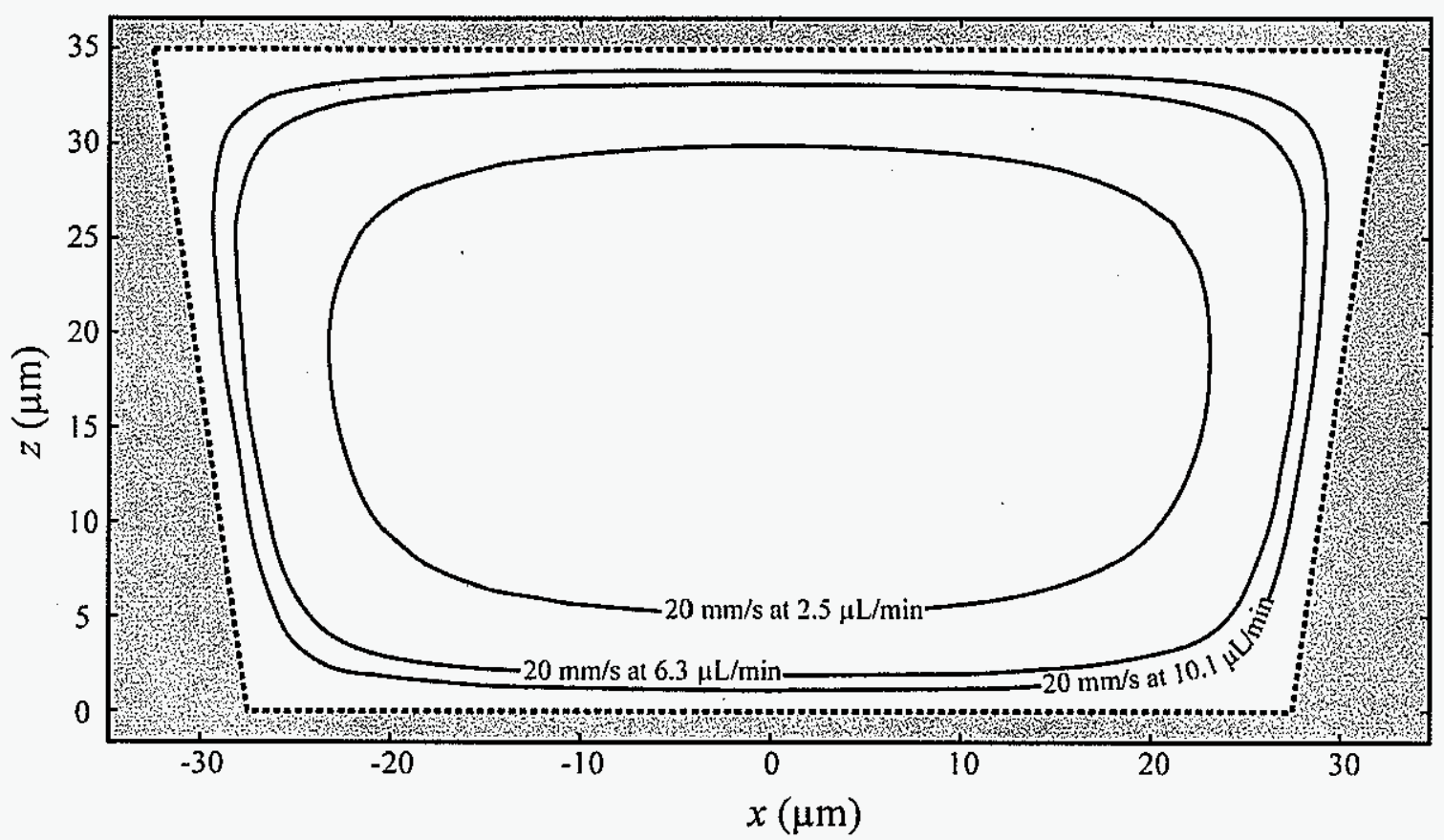

Figure 10. Contours of the $v_{y}=20 \mathrm{~mm} / \mathrm{s}$ flow velocity for three different volumetric flow rates. The dotted line outlines the channel cross-section. Declared values of volumetric flow rates correspond to average flow velocities of 20,50 , and $80 \mathrm{~mm} / \mathrm{s}$, starting from the innermost contour.

\section{Detection of magnetotactic bacteria}

Cells of marine magnetotactic vibrio MV-1 were cultured and then fixed overnight at $4^{\circ}$ $\mathrm{C}$ in $0.1 \%$ glutaraldehyde. The cells were washed three times, resuspended in Tris-borate buffer ( $\mathrm{pH} 8.0$ ), and subsequently stained by adding the Syto 16 fluorescent dye ( $1 \mathrm{mM}$ solution in dimethylsulfoxide) to a final dye concentration of $2 \mu \mathrm{m}$. Fluorescent staining enabled determination of the cell counts using a hemocytometer and a microscope with a $40 \mathrm{x}$ objective lens, and the suspension was diluted with Tris-borate buffer to a final concentration of 30000 cells $/ \mu \mathrm{L}$. The labeled cells appeared well dispersed, with no noticeable aggregates. Three syringe pumps were used to deliver the sample suspension and the two focusing streams (Tris buffer) to the chip. The device was based on the $y$-direction flow layout (perpendicular to the field), and featured bridges with a single sense and three reference $2 \times 2$ $\mu \mathrm{m}$ sensors. Only one of the four bridges was functioning properly. Applied bias voltage was $E_{\text {bias }}=+0.2 \mathrm{~V}$ which required a current of $3.3 \mathrm{~mA}$. The signal was digitized at $100 \mathrm{kHz}$ after 50 -fold amplification.

Because of the misalignment of the fluidic layer relative to sensors, a narrowly focused sample stream would miss the detection volume above the sensor. The flow rates were therefore chosen to produce a relatively wider sample stream that partly flows over the sense 
element. The flow rates were: sample flow rate $\omega_{s}=0.023 \mu \mathrm{L} / \mathrm{min}$, total lateral focus flow rate $\omega_{l}=0.07 \mu \mathrm{L} / \mathrm{min}$, and total vertical focus flow rate $\omega_{\nu}=0.5 \mu \mathrm{L} / \mathrm{min}$. These parameters yield a $9 \times 9 \mu \mathrm{m}$ cross-section of the sample stream. The collected GMR signal is shown in Figure 11. When the lateral focus flow rate was increased to $0.13 \mu \mathrm{L} / \mathrm{min}$ to narrow down the sample stream to about $5 \mu \mathrm{m}$, the GMR signal collapsed to the baseline noise level. This was expected, since under those conditions the sample stream would flow just at the side of the detection volume. The number of detection events, however, yields a concentration estimate of $\sim 5 \times 10^{3}$ cells $/ \mu \mathrm{L}$, at least an order of magnitude greater than the actual concentration. There are several possible reasons for the mismatch: 1) accumulation of the cells at points where capillaries are passing close to electromagnets used to apply the external field, and sporadic release of accumulated cells induced by the fluidic shear forces; (2) flow rate instability due to the limited precision of the syringe pumps used, or the misalignment of the syringes; and (3) sensor noise that mimics the anticipated signal. Further testing is warranted in order to optimize the experimental parameters and fully assess the performance of the platform.
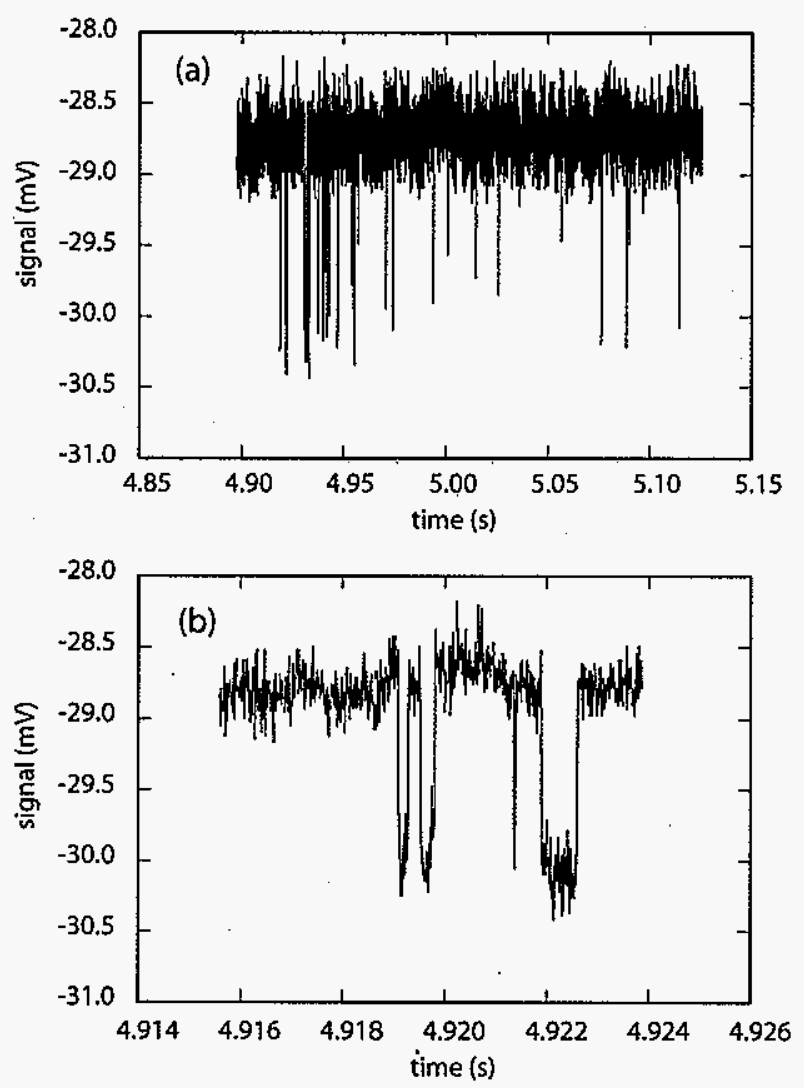

Figure 5.11. (a) A snapshot of the GMR signal recorded during the flow of magnetotactic bacteria. External field strength equaled $1800 \mathrm{~A} \cdot \mathrm{m}^{-1}(22.6 \mathrm{Oe})$. (b) A detail of the same data set. 


\section{Conclusions}

We have designed and fabricated a magnetics-based flow cytometer, and in parallel developed associated numerical models, which establish a set of guidelines for system design and experimental setup. Preliminary experimental findings, based on magnetotactic bacteria as targets, apparently demonstrate single-cell detection events. Further work is needed to substantiate this claim and to fully exploit the potential of the system. In particular, the lack of multiple functioning bridges on the device used in these experiments prevented us from confirming that the observed signal pattern originates from the flow of magnetotactic cells. Repeated experiments with the existing devices are anticipated, and so is an additional fabrication cycle with an improved alignment procedure. The device fabrication, assembly, packaging and inspection are part of a complex operation that involves multiple steps performed at multiple sites, making the logistics somewhat costly and time consuming.

As suggested in the introduction, it would be interesting to explore the applicability of the GMR sensing concept to detection of living magnetotactic cells on chips.

\section{Acknowledgements}

Support from NSF's XYZ-on-a-Chip Initiative (\#88214), the W. M. Keck Foundation, the Institute for Combinatorial Discovery of Iowa State University, and DARPA's BioMagnetICs program is gratefully acknowledged. NP thanks ConocoPhillips for the support of a graduate fellowship. The Ames Laboratory is operated for the U.S. Department of Energy by Iowa State University under contract W-7405-eng-82.

\section{References cited}

1. Sato, H., Schroeder, P. A., Slaughter, J., Pratt, W. P., Jr. \& Abdul-Razzaq, W. Galvanomagnetic properties of silver/M (M = iron, nickel, cobalt) layered metallic films. Superlattices and Microstructures 4, 45-50 (1988).

2. Baibich, M. N. et al. Giant magnetoresistance of (001)iron/(001)chromium magnetic superlattices. Physical Review Letters 61, 2472-5 (1988).

3. Graham, D. L. et al. Magnetic field-assisted DNA hybridization and simultaneous detection using micron-sized spin-valve sensors and magnetic nanoparticles. Sensors and Actuators, B: Chemical B107, 936-944 (2005).

4. Millen, R. L., Kawaguchi, T., Granger, M. C., Porter, M. D. \& Tondra, M. Giant Magnetoresistive Sensors and Superparamagnetic Nanoparticles: A Chip-Scale Detection Strategy for Immunosorbent Assays. Analytical Chemistry 77, 6581-6587 (2005).

5. Li, G. et al. Spin valve sensors for ultrasensitive detection of superparamagnetic nanoparticles for biological applications. Sensors and Actuators, A: Physical A126, 98$106(2006)$. 
6. Rife, J. C. et al. Design and performance of GMR sensors for the detection of magnetic microbeads in biosensors. Sensors and Actuators, A: Physical A107, 209-218 (2003).

7. Pekas, N., Porter, M. D., Tondra, M., Popple, A. \& Jander, A. Giant magnetoresistance monitoring of magnetic picodroplets in an integrated microfluidic system. Applied Physics Letters 85, 4783-4785 (2004).

8. Blakemore, R. Magnetotactic bacteria. Science 190, 377-9 (1975).

9. Bazylinski, D. A. \& Frankel, R. B. Magnetosome formation in prokaryotes. Nature Reviews Microbiology 2, 217-230 (2004).

10. Lee, H., Purdon, A. M., Chu, V. \& Westervelt, R. M. Controlled assembly of magnetic nanoparticles from magnetotactic bacteria using microelectromagnets arrays. Nano Letters 4, 995-998 (2004).

11. Schuler, D. \& Frankel, R. B. Bacterial magnetosomes: microbiology, biomineralization and biotechnological applications. Applied Microbiology and Biotechnology 52, 464-473 (1999).

12. Simmons, S. L., Sievert, S. M., Frankel, R. B., Bazylinski, D. A. \& Edwards, K. J. Spatiotemporal distribution of marine magnetotactic bacteria in a seasonally stratified coastal salt pond. Applied and Environmental Microbiology 70, 6230-6239 (2004).

13. Chemla, Y. R. et al. A new study of bacterial motion: superconducting quantum interference device microscopy of magnetotactic bacteria. Biophysical Journal 76, 33233330 (1999).

14. Tondra, M., Porter, M. \& Lipert, R. J. Model for detection of immobilized superparamagnetic nanosphere assay labels using giant magnetoresistive sensors. Journal of Vacuum Science \& Technology, A: Vacuum, Surfaces, and Films 18, 1125-1129 (2000).

15. Friedman, G. \& Yellen, B. Magnetic separation, manipulation and assembly of solid phase in fluids. Current Opinion in Colloid \& Interface Science 10, 158-166 (2005).

16. Qian, Z., Daughton, J. M., Wang, D. \& Tondra, M. Magnetic design and fabrication of linear spin-valve sensors. IEEE Transactions on Magnetics 39, 3322-3324 (2003).

17. Regenberg, B. et al. Use of laminar flow patterning for miniaturised biochemical assays. Lab on a Chip 4, 654-657 (2004).

18. Hofmann, O., Niedermann, P. \& Manz, A. Modular approach to fabrication of threedimensional microchannel systems in PDMS-application to sheath flow microchips. Lab on a Chip 1, 108-114 (2001). 


\section{CHAPTER 6. GENERAL CONCLUSIONS AND SUGGESTIONS FOR FURTHER RESEARCH}

The cross-disciplinary studies presented in this dissertation illustrate a remarkable prospective that micromagnetic tools bring into the lab-on-a-chip arena. We have demonstrated, in a microfluidic format, in-flow manipulation of magnetic particles, integrated detection and multiparametric characterization of micrometer-sized droplets of magnetic fluids, and detection of magnetic particles and magnetotactic bacteria. While central motivation for our efforts is the development of a miniaturized magnetic flow cytometer, the implications span a broader set of applications, from magnetic nanoparticle analysis to cell motility assessment. The outlook is exciting, and it encompasses several opportunities for further progress that will be briefly discussed.

\section{Device fabrication}

The fabrication of complex, multilayered devices has proven difficult at times. The sensitivity, dynamic range, and working point of sensors can be further optimized to enhance the detection efficacy. A precise relative alignment of sensors and microfluidic channels is of utmost importance for the hydrodynamic focusing concept detailed in Chapter 5. The required precision is defined by the sensor size, and for a $2 \times 2 \mu \mathrm{m}$ sensor should be better than $1 \mu \mathrm{m}$. Such precision is somewhat challenging, yet achievable with conventional contact mask aligners. One way to circumvent the present fluidic-electronic alignment problem is to deploy an additional focusing mode that is based on electromagnetics rather than hydrodynamics. The electromagnetic "railroad" structures for lateral focusing, based partly on our studies described in Chapter 2, are included in the existing design and fabricated in the same metal layer that defines the sensor interconnects, ensuring precise positioning relative to sensors. A likely side-effect of the deployment of such current lines would be the accumulation of targets due to attractive forces acting toward the channel bottom, but careful optimization of flow rates and the use of pulsed current might alleviate this problem.

\section{The role of intrinsic fields}

An electrical current flowing through a conducting structure will result in a magnetic field surrounding the conductor, as described by the Biot-Savart law. ${ }^{1}$ In Chapter 2 , we utilized such fields to manipulate particles in microchannels above current lines. The same consideration applies to GMR sensors themselves - a local field will result from the sensor current. Additionally, the magnetostatic fields of the pinned and the free layers of the sensor 
will contribute to the overall field profile around the sensor. ${ }^{2}$ In Chapter 3 , we noted the ability to observe the magnetic signal even without an external excitation field - an effect attributed to the fields induced by the sense current flowing through the pairs of sense GMR elements. In a recent article, ${ }^{3}$ Ferreira et al. suggest that the intrinsic fields indeed are important, and have to be taken into account when designing experiments and interpreting data.

\section{Hydrodynamic lift forces}

The field of a dipole decays with the third power of distance, ${ }^{1}$ mandating control of target-sensor separation. When detecting targets in flow, hydrodynamic lift forces ${ }^{4}$ contest the need to minimize the target separation from the sensor. The theory of hydrodynamic lift in microfluidic channels is based on semi-empirical notions and numerical modeling, and provides little, if any, information on behavior of irregular objects such as cells. Estimates based on theoretical ${ }^{5}$ and experimental ${ }^{6}$ studies place the lift force on a $2-\mu \mathrm{m}$ spherical particle flowing close to the channel wall in the range of $10^{-13}-10^{-12} \mathrm{~N}$. The force of $10^{-12} \mathrm{~N}$, using the equations derived in Chapter 2, corresponds to a lift velocity of $50 \mu \mathrm{m} / \mathrm{s}$. Assuming an average flow velocity of $10 \mathrm{~mm} / \mathrm{s}$, the particle would migrate about $1 \mu \mathrm{m}$ per each $200 \mu \mathrm{m}$ of the flow path. This estimate suggests that the lift force, although not overly detrimental, should be included in the design and detailed analysis of the flow focusing system.

\section{Magnetic properties of labels, and magnetic and viscous torque}

A number of commercially available magnetic markers have been developed with the separation applications in mind, and devising particles more appropriate for use as reporters may be possible. An ideal magnetic label for the flow cytometric applications would be based on monodisperse, nanoscale superparamagnetic or ferromagnetic particles. In both cases, the particles should be chemically and colloidally stable, and have a well-defined coupling chemistry for conjugation to antibodies or nucleic acids.

Superparamagnetic labels, due to the absence of the magnetization when there is no externally applied field, are generally more stable against aggregation than their ferromagnetic counterparts. Since magnetic moments of intrinsically superparamagnetic particles realign instantaneously with an external field, they can accommodate any rotation of labeled targets in shear flows without the changes in observed signal. On the other hand, currently available iron-oxide based superparamagnetic particles provide only moderate magnetic moments at the operating fields of spin valve sensors, as shown in Chapter 4. It is 
likely that metallic or alloy-based particles, such as FeNi, would show much better magnetic performance, ${ }^{7}$ but chemical stability and bioconjugation chemistry problems are still to be addressed in this case.

Ferromagnetic labels are attractive as reporters, since their relatively high magnetic moments are preserved at low operating fields. Upon binding demagnetized labels to a cell, a brief exposure to a uniform and sufficiently strong magnetic field would magnetize the labels prior to detection. As is evident from the numerical models presented in Chapter 4, a magnetized ferromagnetic bead would exhibit 20 to 100 times stronger magnetic signature than the superparamagnetic bead of a comparable size, depending on the sensor size and separation from the bead. From the magnetic flow cytometry point of view, however, ferromagnetic labels require an extensive analysis of the target rotation phenomena due to the viscous torque in shear flows in microchannels; ${ }^{5,8}$ this is an important consideration because our magnetic detection design assumes that the magnetic moment of the target is aligned with the sense direction of GMR strips.

A limited number of studies are available that address the fluidic torque on non-spherical objects in flow. ${ }^{5,8,9}$ A general conclusion pertaining to the case of vertical hydrodynamic confinement is that a spheroid, a rod, or a disc will rotate at the rate that depends on the rotation angle at any point of time. For certain shapes and aspect ratios, the object may reach a stable orientation, usually such that the long dimension is aligned with the flow direction. In other cases, the object will tumble at a non-uniform rate, but the probability of finding the object aligned with the flow direction will be higher than for other orientations. Based on the work by Gavze et al., ${ }^{9}$ it can be estimated that a maximum torque on a prolate spheroid of $3 \times 1 \mu \mathrm{m}$ (for a $\theta=90^{\circ}$ angle between the flow direction and the long axis of the spheroid) would be around $6 \times 10^{-17} \mathrm{~N} \cdot \mathrm{m}$. Similarly, the results from Kuzhir et al. suggest a maximum torque on a $3 \times 1 \mu \mathrm{m}$ rod of around $2 \times 10^{-17} \mathrm{~N} \cdot \mathrm{m} .{ }^{10}$ In both cases, the torque obeys a squaredsine dependence on the orientation angle $\theta$, approaching a zero value when the objects are aligned with the flow direction.

In typical magnetotactic bacteria, the magnetic moment of the magnetosome is aligned with the long axis of the cell. ${ }^{11}$ When an external magnetic field is applied parallel to the direction of the flow, the magnetic torque (as defined in Chapter 1) will work in accord with the viscous torque to align the cell with the flow. A maximum magnetic torque on a magnetotactic bacterium (for a $\theta=90^{\circ}$ angle between the magnetic field and the magnetic moment of the cell) would be around $10^{-18} \mathrm{~N} \cdot \mathrm{m}$. Being a vector product of the magnetic moment and the field inductance, the magnetic torque intensity will be proportional to the sine of the angle between these two vectors. For small deviations from the fully aligned state, 
the magnetic torque will prevail over viscous torque (sine vs. squared sine dependence) and effectively lock in the orientation at $\theta=0^{\circ}$. The situation will be quite different when the external field is applied perpendicular to the flow direction - the two torques then become orthogonal. It is uncertain at this stage as to how stable the cell orientation would be in this case, and further research on this topic would be helpful. Given the mathematical complexity of the problem and the distribution of cell shapes and sizes, the first step in addressing the orientation problem would be empirical: carefully designed microscopical investigations may provide valuable information about the cell paths and orbits within microchannels.

For cellular targets other than magnetotactic bacteria, the shape may be more or less spherical, and the overall magnetic moment of the attached labels is not necessarily related to the cell shape in any way. The viscous torque for more spherical objects becomes less dependant on the orientation angle and therefore becomes constant, ${ }^{9}$ and a sufficient amount of ferromagnetic labels will be required to guarantee that the magnetic torque keeps the magnetic moment of the cell aligned against any perturbation by viscous forces.

\section{References cited}

1. Chikazumi, S. Physics of magnetism (Wiley, New York, 1964).

2. Li, G. et al. Spin valve sensors for ultrasensitive detection of superparamagnetic nanoparticles for biological applications. Sensors and Actuators, A: Physical A126, 98$106(2006)$.

3. Ferreira, H. A., Feliciano, N., Graham, D. L. \& Freitas, P. P. Effect of spin-valve sensor magnetostatic fields on nanobead detection for biochip applications. Journal of Applied Physics 97, 10Q904/1-10Q904/3 (2005).

4. Williams, P. S., Lee, S. \& Giddings, J. C. Characterization of hydrodynamic lift forces by field-flow fractionation. Inertial and near-wall lift forces. Chemical Engineering Communications 130, 143-66 (1994).

5. Zhao, Y. \& Sharp, M. K. Stability of elliptical cylinders in two-dimensional channel flow. Journal of biomechanical engineering 122, 493-7 (2000).

6. Williams, P. S., Koch, T. \& Giddings, J. C. Characterization of near-wall hydrodynamic lift forces using sedimentation field-flow fractionation. Chemical Engineering Communications 111, 121-47 (1992).

7. Rife, J. C. et al. Design and performance of GMR sensors for the detection of magnetic microbeads in biosensors. Sensors and Actuators, A: Physical A107, 209-218 (2003).

8. Mason, S. G. \& Goldsmith, H. L. in Circulatory and Respiratory Mass Transport (eds. Wolstenholme, G. E. W. \& Knight, J.) 105-129 (Little, Brown and Company, Boston, 1969). 
9. Gavze, E. \& Shapiro, M. Particles in a shear flow near a solid wall: effect of nonsphericity on forces and velocities. International Joumal of Multiphase Flow 23, 155 182 (1997).

10. Kuzhir, P., Bossis, G. \& Bashtovoi, V. Effect of the orientation of the magnetic field on the flow of a magnetorheological fluid. I. Plane channel. Journal of Rheology (New York, NY, United States) 47, 1373-1384 (2003).

11. Schuler, D. \& Frankel, R. B. Bacterial magnetosomes: microbiology, biomineralization and biotechnological applications. Applied Microbiology and Biotechnology 52, 464-473 (1999). 